\title{
Deconfined Quantum Critical Points: Symmetries and Dualities
}

\author{
Chong Wang, ${ }^{1,2}$ Adam Nahum, ${ }^{3,4}$ Max A. Metlitski, ${ }^{3,5,2}$ Cenke Xu, ${ }^{6,2}$ and T. Senthil ${ }^{3}$ \\ ${ }^{1}$ Department of Physics, Harvard University, Cambridge, Massachusetts 02138, USA \\ ${ }^{2}$ Kavli Institute for Theoretical Physics, University of California, Santa Barbara, California 93106, USA \\ ${ }^{3}$ Department of Physics, Massachusetts Institute of Technology, Cambridge, Massachusetts 02139, USA \\ ${ }^{4}$ Theoretical Physics, Oxford University, 1 Keble Road, Oxford OX1 3NP, United Kingdom \\ ${ }^{5}$ Perimeter Institute for Theoretical Physics, Waterloo, Ontario N2L 2Y5, Canada \\ ${ }^{6}$ Department of Physics, University of California, Santa Barbara, California 93106, USA \\ (Received 4 May 2017; revised manuscript received 25 July 2017; published 22 September 2017)
}

The deconfined quantum critical point (QCP), separating the Néel and valence bond solid phases in a 2D antiferromagnet, was proposed as an example of $(2+1) \mathrm{D}$ criticality fundamentally different from standard Landau-Ginzburg-Wilson-Fisher criticality. In this work, we present multiple equivalent descriptions of deconfined QCPs, and use these to address the possibility of enlarged emergent symmetries in the lowenergy limit. The easy-plane deconfined QCP, besides its previously discussed self-duality, is dual to $N_{f}=2$ fermionic quantum electrodynamics, which has its own self-duality and hence may have an $\mathrm{O}(4) \times Z_{2}^{T}$ symmetry. We propose several dualities for the deconfined QCP with $\mathrm{SU}(2)$ spin symmetry which together make natural the emergence of a previously suggested $\mathrm{SO}(5)$ symmetry rotating the Néel and valence bond solid orders. These emergent symmetries are implemented anomalously. The associated infrared theories can also be viewed as surface descriptions of $(3+1) \mathrm{D}$ topological paramagnets, giving further insight into the dualities. We describe a number of numerical tests of these dualities. We also discuss the possibility of "pseudocritical" behavior for deconfined critical points, and the meaning of the dualities and emergent symmetries in such a scenario.

DOI: 10.1103/PhysRevX.7.031051

Subject Areas: Condensed Matter Physics,
Particles and Fields,
Statistical Physics

\section{INTRODUCTION}

Zeus, the ruler of the Olympian gods, often conceals his identity by changing himself into different forms. Strongly interacting conformal field theories (CFTs), which underlie many different states of matter, can sometimes also be described by Lagrangians with very different forms. In other words, two seemingly different "dual Lagrangians may correspond to the same CFT. The classic example of such a duality is the equivalence between the $3 \mathrm{D} \mathrm{O}(2)$ Wilson-Fisher fixed point and the Higgs transition of bosonic quantum electrodynamics (QED) with one flavor of complex boson [1-3]. Either theory describes interacting lattice bosons at the quantum phase transition between a superfluid phase, in which U(1) symmetry is spontaneously broken, and a Mott insulating phase, in which it is not.

This paper studies dualities for quantum phase transitions in two spatial dimensions that lie outside the Landau paradigm. Our focus is on non-Landau transitions between two conventional phases, each of which is well described

Published by the American Physical Society under the terms of the Creative Commons Attribution 4.0 International license. Further distribution of this work must maintain attribution to the author(s) and the published article's title, journal citation, and DOI. by a Landau order parameter. The paradigmatic example of such a phase transition occurs in two-dimensional quantum magnets. Square lattice spin-1/2 magnets allow (as the interactions are changed) a conventional Néel antiferromagnetic phase which breaks spin-rotation symmetry and a valence bond solid (VBS) phase: a crystal of spin singlets which preserves the spin-rotation symmetry while breaking lattice symmetries. A field theory for a putative continuous phase transition between the Néel and VBS phases was described in Refs. [4,5]. The theory-known as the noncompact $\mathrm{CP}^{1}$ model $\left(\mathrm{NCCP}^{1}\right)$ is formulated in terms of fractionalized "spinon" degrees of freedom [a bosonic field $z_{\alpha}$, with $\alpha=1,2$ an $\mathrm{SU}(2)$ flavor index] coupled to a noncompact $\mathrm{U}(1)$ gauge field $b$. Neither the spinon nor the gauge photon, however, exist as deconfined quasiparticles in either phase. The phase transition has, hence, been dubbed a "deconfined quantum critical point." Numerical work on specific quantum magnets and related systems [6-22] shows a striking (apparently [23]) continuous phase transition with properties broadly consistent with fieldtheoretic expectations. The deconfined criticality scenario also generalizes to $\mathrm{SU}(N)$ magnets with large $N$, where there is a second-order phase transition that is under good theoretical control.

Analytic progress on the field theories for SU(2) deconfined quantum critical points [27] has been challenging. 
References [28,29] showed that a formulation directly in terms of the Landau order parameters for the two phases was possible using a nonlinear sigma model, but required the addition of a "topological" term to correctly capture their competition or intertwinement. This term endows the topological defects of each order parameter with nontrivial symmetry properties, enabling the Landau-forbidden phase transition. This sigma model formulation gave rise to the possibility that the phase transition may have a large emergent symmetry, which rotates the two Landau order parameters (Néel and VBS) into each other.

Remarkably, recent numerical work finds evidence for the emergence of such a higher symmetry. Specifically, a model described by $\mathrm{NCCP}^{1}$ [a field theory which naively has only $\mathrm{SO}(3) \times \mathrm{O}(2)$ symmetry] was seen to have an emergent $\mathrm{SO}(5)$ symmetry at the critical point, at the length scales accessible in the calculations [15]. A good understanding of this emergent extra symmetry is currently not available.

In a different direction, many fascinating new dualities for field theories with $\mathrm{U}(1)$ gauge fields have been found very recently [30-37]. These dualities originated from studies of the surface of three-dimensional symmetryprotected topological (SPT) phases [30,31,33], their relation to three-dimensional quantum spin liquids with an emergent $\mathrm{U}(1)$ gauge field $[31,38,39]$, and the physics of the half-filled Landau level of two-dimensional electrons [40-42]. Other related dualities were discussed in a highenergy context (see Ref. [43] and references therein).

From a modern point of view, the classic infrared duality between the $\mathrm{O}(2)$ Wilson-Fisher theory and bosonic QED is natural because the two field theories have the same global symmetry, namely, U(1), the same allowed quantum numbers for gauge-invariant local operators (here, bosons of integer charge). and the same anomalies (none in this case). The two ultraviolet Lagrangians can therefore be viewed as descriptions of the same physical system with different bare interactions, making it possible that their long-distance behavior is the same. The dualities mentioned above are extensions of this idea to situations in which the operator content and anomalies are nontrivial. In this paper, we apply this philosophy to the field theories for deconfined critical points.

We propose and analyze dualities involving these field theories, paying special attention to the realization of symmetries. These dual descriptions give a new way of understanding emergent symmetries relating the Landau order parameters of the Néel and VBS phases.

For the easy-plane version of the $\mathrm{NCCP}^{1}$ model (describing the Néel-VBS transition in magnets with $X Y$ spin symmetry) several dual descriptions have been discussed in the old and recent literature, as we review below. Some of these dual theories are formulated in terms of bosonic fields while others involve fermionic fields. These boson or fermion fields are coupled to a dynamical U(1) gauge field. Here, we unify these different dual descriptions into a common duality web and clarify the emergent symmetries of the putative critical fixed point.

For the $\mathrm{SU}(2)$-symmetric $\mathrm{NCCP}^{1}$ model, we propose a dual fermionic description as massless $\mathrm{QED}_{3}$ coupled to a critical real scalar field. We refer to this theory as $\mathrm{QED}_{3^{-}}$ Gross-Neveu (GN). We show that this duality implies the emergent $\mathrm{SO}(5)$ symmetry at this deconfined critical point (as observed in numerical simulations). We are then led to propose a duality web for the $\mathrm{SU}(2)$-symmetric $\mathrm{NCCP}^{1}$ theory as well. The existence of this duality web provides an alternate point of view on the emergence of the $\mathrm{SO}(5)$ symmetry at the deconfined critical point.

Remarkably, the duality web implies that the SU(2)symmetric $\mathrm{NCCP}^{1}$ model is itself self-dual. Indeed, if we assume this self-duality, the $\mathrm{SO}(5)$ symmetry follows as an inescapable consequence. Conversely, the existing evidence for the emergent $\mathrm{SO}(5)$ symmetry strongly supports the conjectured self-duality of the $\mathrm{SU}(2)$-symmetric $\mathrm{NCCP}^{1}$ model.

We show that useful insight into these field theories is obtained by realizing them at the boundary of $(3+1) \mathrm{D}$ bosonic symmetry-protected topological phases. This allows the theories to be regularized in a way that preserves the full internal symmetry of the putative IR fixed point. By contrast, the full internal symmetry of the IR theory cannot be incorporated microscopically in a strictly 2D quantum magnet: it can only be emergent. In field-theoretic parlance, the symmetry of the IR theory is anomalous, and the anomaly is canceled when the theory resides at the boundary of a $(3+1) \mathrm{D}$ SPT phase. Furthermore, in the easy-plane case, we show how the "bulk" $(3+1) \mathrm{D}$ description provides a very simple explanation for the existence of the duality web and the symmetry realizations of the various theories contained therein. For the SU(2)invariant case, with its putative emergent $\mathrm{SO}(5)$ symmetry, we describe a manifestly $\mathrm{SO}(5)$-invariant formulation in terms of massless fermions coupled to an $\mathrm{SU}(2)$ gauge field, a theory we denote $N_{f}=2 \mathrm{QCD}_{3}$. This $(2+1) \mathrm{D}$ theory is shown to have the same anomaly as the proposed $\mathrm{SO}(5)$-invariant fixed point associated with the $\mathrm{NCCP}^{1}$ theory. This allows us to show that there is a corresponding bulk SPT phase of bosons with global $\mathrm{SO}(5)$ symmetry [i.e., an $\mathrm{SO}(5)$ "topological paramagnet"]. This boson SPT is characterized in the bulk by its response to an external background $\mathrm{SO}(5)$ gauge field. This response includes a nontrivial discrete theta angle, introduced in Ref. [44], which distinguishes it from a trivial gapped phase of $\mathrm{SO}(5)-$ symmetric bosons. The $(2+1) \mathrm{D}$ theories with anomalous $\mathrm{SO}(5)$ symmetry are alternative descriptions of the surface of this $(3+1)$ D boson SPT phase.

It is important to distinguish two different versions of statements about duality of quantum field theories that are conflated in the literature. First, there are "weak" duality statements. These assert that the two theories in question 
have the same local operators, the same symmetries, and the same anomalies (if any). In condensed-matter parlance, this means that the two theories "live in the same Hilbert space" and can be viewed as descriptions of the same microscopic system in different limits. These weak dualities are nontrivial statements that can be unambiguously derived. In the context of the present paper their main interest is that they open up the possibility of "strong" dualities. The strong dualities will hold if the putatively dual theories flow, without fine-tuning, to the same nontrivial IR fixed point. For the theories we discuss here, it is these strong dualities that would imply the emergence of large, exact symmetries in the infrared. We do not derive the strong dualities in this paper, but rather view them as plausible conjectures suggested by the weak dualities.

In fact, the strong dualities can be relevant to the physics up to a very large length scale even in the absence of a true fixed point, if the system shows quasiuniversal "pseudocritical" behavior up to a large length scale. We emphasize that it is not yet clear whether the theories we discuss do flow to nontrivial IR fixed points: this is an ongoing question for numerical work. But for the SU(2)-symmetric $\mathrm{NCCP}^{1}$ model, simulations show that there is at least apparent critical behavior up to a remarkably large length scale. Numerical evidence for $\mathrm{SO}(5)$ in this regime supports the applicability of the $\mathrm{SO}(5)$ web of dualities. For $\mathrm{QED}_{3}$, very recent simulations argued for a flow to a conformal fixed point [45-47], while earlier studies argued for (very weak) chiral symmetry breaking [48]. For the easy-plane $\mathrm{NCCP}^{1}$ model the current numerical consensus is that the transition is weakly first order. The duality to $\mathrm{QED}_{3}$ suggests that it may be worth revisiting the Néel-VBS transition in easy-plane magnets and related models to look for a second-order transition.

We describe consequences of the strong duality conjectures that may be tested in future numerical work. Our proposed duality web for $\mathrm{SU}(2)$-invariant $\mathrm{NCCP}^{1}$ and $\mathrm{QED}_{3}$-Gross-Neveu involves an emergent $\mathrm{SO}(5)$ symmetry, and leads to clear and testable predictions for the behavior of two-flavor $\mathrm{QED}_{3}$ when it is coupled to a critical real scalar field. The web of dualities involving easy-plane $\mathrm{NCCP}^{1}$ and two-flavor $\mathrm{QED}_{3}$ are naturally thought of in terms of a "mother" theory with an $\mathrm{O}(4)$ symmetry which rotates the Néel and VBS order parameters. For $\mathrm{QED}_{3}$ this emergent symmetry should have striking numerically accessible consequences. Our results also show how numerical and analytical studies of $\mathrm{QED}_{3}$ and $\mathrm{QED}_{3}$ - $\mathrm{GN}$ will provide new information about deconfined criticality.

The duality transformations we employ involve global symmetries with a $\mathrm{U}(1)$ subgroup. For a $(2+1) \mathrm{D}$ CFT with a global $U(1)$ symmetry there are two basic formal transformations-denoted $S$ and $T$-which map the theory to other inequivalent theories with a global U(1) symmetry, assumed also to be CFTs $[49,50]$. Our duality transformations can be viewed within this framework. However, there are a number of caveats about the standard use of the $S$ and $T$ transformations that we discuss in Appendix C. Making standard assumptions about the effect of $S$ and $T$ on CFTs allows stronger assertions about deconfined critical points and their symmetries than those discussed above. However, it is not clear at this point whether these standard assumptions can be trusted far from the context in which they were originally discussed, i.e., in nonsupersymmetric theories that are far from any large- $N$ limit.

\section{PRELIMINARIES AND SUMMARY OF RESULTS}

\section{A. Deconfined quantum criticality: $\mathrm{NCCP}^{1}$ and related models}

We first briefly recall the theory of deconfined quantum critical points in quantum magnets. For a spin-1/2 quantum antiferromagnet on a two-dimensional square lattice, the transition between the Néel ordered magnet and the VBS paramagnet is potentially second order and is described by the $\mathrm{NCCP}^{1}$ field theory:

$$
\mathcal{L}_{0}=\sum_{\alpha=1,2}\left|D_{b} z_{\alpha}\right|^{2}-\left(\left|z_{1}\right|^{2}+\left|z_{2}\right|^{2}\right)^{2} .
$$

Here, $z_{\alpha}(\alpha=1,2)$ are bosonic spinons coupled to a dynamical U(1) gauge field $b$, and $D_{b, \mu}=\partial_{\mu}-i b_{\mu}$ is the covariant derivative. (This action and subsequent similar actions are shorthand for the appropriate strongly coupled Wilson-Fisher critical theory where a background gauge field has been promoted to a dynamical field; unless otherwise specified, they are written in Minkowski signature.) The model has a global $\mathrm{SO}(3)$ symmetry under which $z_{\alpha}$ transforms as a spinor. [51] In the microsopic lattice spin model, this corresponds to the $\mathrm{SO}(3)$ spin rotation. It also has a global U(1) symmetry associated with the conservation of the flux [52] of $b$. In the microsopic lattice spin model, this is not an exact symmetry. Consequently, monopole operators [which pick up a phase under a $\mathrm{U}(1)$ rotation] must be added to the Lagrangian. However, it is known that lattice symmetries ensure that the minimal allowed monopole operator (with continuum angular momentum $\ell=0$ ) has strength 4 . Analytic arguments $[4,5]$ and numerical calculations $[6,8,14]$ strongly support the possibility that these monopoles are irrelevant at the critical fixed point of Eq. (1). The Néel phase is obtained when $z_{\alpha}$ is condensed, and the VBS phase when $z_{\alpha}$ is gapped. The Néel phase breaks $\mathrm{SO}(3)$ to a $\mathrm{U}(1)$ subgroup while the VBS phase breaks the U(1) flux conservation symmetry. The Néel order parameter is simply $\mathbf{N}=z^{\dagger} \sigma z$ ( $\sigma$ are Pauli matrices), and the VBS order parameter is the strength-1 monopole operator $\mathcal{M}_{b}$ which creates $2 \pi$ flux of $b$.

If the underlying spin model has only $\mathrm{O}(2)(X Y)$ spin symmetry-corresponding to conservation of the $z$ 
component of spin, together with a discrete $\pi$ rotation of the spins around the $x$ axis, which we denote $\mathcal{S}$ - then the NéelVBS phase transition is described by the theory

$$
\mathcal{L}_{e p-c p 1}=\sum_{\alpha=1,2}\left|D_{b} z_{\alpha}\right|^{2}-\left(\left|z_{1}\right|^{4}+\left|z_{2}\right|^{4}\right)+\cdots .
$$

This is known as the easy-plane $\mathrm{NCCP}^{1}$ model. In this model the $X Y$ Néel order parameter is $N_{x}+i N_{y}=2 z_{1}^{*} z_{2}$, while the VBS order parameter is the monopole operator $\mathcal{M}_{b}$. Note that under the $Z_{2}$ spin-flip symmetry $\mathcal{S}$, we have

$$
\mathcal{S}: z \rightarrow \sigma_{x} z, \quad b \rightarrow b .
$$

Then, under $\mathcal{S}$ the $X Y$ Néel order parameter transforms as $N_{x}+i N_{y} \rightarrow N_{x}-i N_{y}$ and the VBS order parameter $\mathcal{M}_{b}$ is invariant, as expected microscopically. Later in the paper we describe the action of time-reversal and lattice symmetries for square lattice antiferromagnets (Sec. III B).

The easy-plane theory is known to be self-dual [53], in the sense that it is dual to another easy-plane $\mathrm{NCCP}^{1}$ theory,

$\mathcal{L}_{e p-c p 1-\text { dual }}=\sum_{\alpha=1,2}\left|D_{\tilde{b}} w_{\alpha}\right|^{2}-\left(\left|w_{1}\right|^{4}+\left|w_{2}\right|^{4}\right)+\cdots$,

in which the roles of the two order parameters are switched: $w_{1}^{*} w_{2}$ is the VBS order parameter, while $\mathcal{M}_{\tilde{b}}$ is the $X Y$ order parameter. This self-duality is obtained by applying the particle-vortex duality to both spinons: $z_{1} \rightarrow w_{2}$, $z_{2} \rightarrow w_{1}^{*}$. Since the boson mass term is odd under the particle-vortex duality, the self-duality sends $\left|z_{1}\right|^{2} \rightarrow-\left|w_{2}\right|^{2}$ and $\left|z_{2}\right|^{2} \rightarrow-\left|w_{1}\right|^{2}$.

The IR fates of the two $\mathrm{NCCP}^{1}$ models, and their generalizations with an $N$-component spinon field $z_{\alpha}$, have been discussed extensively. They flow to conformal field theories within a $1 / N$ expansion. Directly at $N=2$, numerical calculations see an apparently continuous transition in the $\mathrm{SU}(2)$-invariant $\mathrm{NCCP}^{1}$ model, but with drifts in some critical properties (which we discuss in Sec. IX). Further recent studies show the emergence of an $\mathrm{SO}(5)$ symmetry that rotates the Néel and VBS order parameters into one another. For the easy-plane case, the current wisdom is that the Néel-VBS transition is weakly first order. However, as we discuss at length, the potential duality with $\mathrm{QED}_{3}$ may make it interesting to examine this further.

These gauge theories give a natural route to a secondorder transition between two distinct symmetry-broken phases, despite the fact that such a transition is naively forbidden by the Landau theory. In contrast to the standard Landau-Ginzburg-Wilson description, the critical theory is expressed in terms of "deconfined" degrees of freedom (the spinons and the gauge field) which do not describe sharp quasiparticles in either phase. Physically the breakdown of the Landau paradigm occurs because the topological defects of either order parameter carry nontrivial quantum numbers: the Skyrmion defect of the Néel phase carries quantum numbers under lattice symmetries [4,5,54,55], and the vortex defect of the VBS phase carries spin 1/2 [56].

There is an alternative formulation [28,29] for the competition between the two order parameters directly in terms of a nonlinear sigma model. In the SU(2)-invariant case, we define a real five-component unit vector $n^{a}$ $(a=1, \ldots, 5)$ such that $n^{3,4,5}$ correspond to the three components of the Néel vector, and $n^{1,2}$ to the two real components of the VBS order parameter. The intertwined fluctuations of the two order parameters are then described by an $\mathrm{SO}(5)$ action with a Wess-Zumino-Witten (WZW) term at level 1:

$$
S=\frac{1}{2 g} \int d^{3} x\left(\partial n^{a}\right)^{2}+2 \pi \Gamma\left[n^{a}\right] .
$$

The WZW term $\Gamma$ is defined in the standard way: the field $n^{a}$ defines a map from spacetime $S^{3}$ to the target space $S^{4}$, and $\Gamma$ is the ratio of the volume in $S^{4}$ traced out by $n_{a}$ to the total volume of $S^{4}$. If $n^{a}(x, u)$ is any smooth extension of $n^{a}(x)$ such that $n^{a}(x, 0)=(0,0,0,0,1)$ and $n^{a}(x, 1)=$ $n^{a}(x)$, then

$$
\Gamma=\frac{\epsilon_{a b c d e}}{\operatorname{area}\left(S^{4}\right)} \int_{0}^{1} d u \int d^{3} x n^{a} \partial_{x} n^{b} \partial_{y} n^{c} \partial_{t} n^{d} \partial_{u} n^{e} .
$$

In order to share the symmetry of the $\mathrm{NCCP}^{1}$ model, the above action must also be supplemented with anisotropy terms that break $\mathrm{SO}(5)$ to $\mathrm{SO}(3) \times \mathrm{U}(1)$. The $\mathrm{WZW}$ term correctly captures the nontrivial quantum numbers of the topological defects and is responsible for the non-Landau physics. For example, if the U(1) symmetry is spontaneously broken, a vortex in the $\mathrm{U}(1)$ order parameter will carry spin $1 / 2$ under the unbroken $\mathrm{SO}(3)$.

The easy-plane case can be obtained from this theory by setting $n^{5}=0$. This then leads to an $\mathrm{O}(4)$ nonlinear sigma model in $2+1$ spacetime dimensions supplemented with a $\theta$ term at $\theta=\pi$ :

$S=\int d^{3} x\left[\frac{1}{2 g}\left(\partial n^{a}\right)^{2}+\frac{\theta \epsilon_{a b c d}}{\operatorname{area}\left(S^{3}\right)} n^{a} \partial_{t} n^{b} \partial_{x} n^{c} \partial_{y} n^{d}\right]$.

The value $\theta=\pi$ is robust as a result of the $\mathbb{Z}_{2}$ spin-flip symmetry $\mathcal{S}$ of the easy-plane $\mathrm{NCCP}^{1}$ model, which changes the sign of $n_{5}$ and therefore acts as $\theta \rightarrow-\theta$. This topological term is once again responsible for the nontrivial structure of the topological defects.

The sigma model formulation raises the possibility that the phase transition described by Eq. (1) may have an emergent $\mathrm{SO}(5)$ symmetry [O(4) in the easy-plane case]. However, we should emphasize that the sigma model is well defined as a continuum field theory only in the weak coupling limit, where it is ordered. Here, there is a clear 
semiclassical picture for the effect of the WZW term [ $\theta$ term in the $\mathrm{O}(4)$ case] on the topological defects in the ordered state. For the transition itself-driven by anisotropy for the $\mathrm{SO}(5)$ or $\mathrm{O}(4)$ vector - this ordered state corresponds to a first-order phase transition. To study second-order Landau-forbidden transitions, we need to extend the model to strong coupling, and look for a disordered but power-law correlated SO(5)-invariant fixed point. [57] At strong coupling the sigma model theory is nonrenormalizable and requires an alternative formulation as a continuum field theory. Physically, disordered phases of the sigma model (defined with an explicit UV cutoff) correspond to phases where topological defects of the order parameter have proliferated. Thus, a modification of the topological defects leads to modifications of the corresponding disordered phases. The sigma model formulation thus exposes the seed, in the ordered phase, of the impending non-Landau physics of the disordered critical regime.

Yet another formulation $[28,29]$ of the intertwinement of the Néel and VBS orders that maintains manifest $\mathrm{SO}(5)$ symmetry may be obtained by starting with a fermionic spinon description of the square lattice spin-1/2 magnet. This naturally leads to a low-energy theory of two flavors of massless Dirac fermions coupled to a dynamical SU(2) gauge field—a theory we denote $N_{f}=2 \mathrm{QCD}_{3}$. This theory will be useful for some purposes: we discuss it further in Secs. VI and VII.

Finally, deconfined critical field theories also arise in the context of phase transitions between trivial and SPT phases $[46,47,58]$. We review this connection as needed later in the paper.

\section{B. Fermionic $N_{f}=2 \mathrm{QED}_{3}$ and related models}

We now turn our attention to fermionic massless $\mathrm{QED}_{3}$ models with $N_{f}=2$ flavors of two-component fermions [59]:

$$
\mathcal{L}_{\mathrm{QED}}=\sum_{j=1}^{2} i \bar{\psi}_{j} \not_{a} \psi_{j}+\cdots,
$$

where $D_{a}=\gamma^{\mu} D_{a, \mu}$ is the gauge covariant Dirac operator that involves a dynamical noncompact U(1) gauge field $a_{\mu}$ (we choose $\gamma^{0,1,2}=\left\{\sigma^{y}, i \sigma_{z}, i \sigma_{x}\right\}$ and $\bar{\psi}=\psi^{\dagger} \gamma^{0}$ ). The flavor symmetry of the model will play an important role in our discussion. We often, but not always, restrict attention to the case with symmetry under $\mathrm{SU}(2)$ rotations between the two flavors. In addition, there is a global U(1) symmetry associated with the conservation of the flux of the gauge field $a$. The theory then has manifest global $\left\{[\mathrm{SU}(2) \times \mathrm{U}(1)] / Z_{2}\right\}$ symmetry. [61] (The full manifest symmetry of the field theory is larger once charge conjugation is included. [62]) It is sometimes, however, convenient to consider a more general class of $\mathrm{QED}_{3}$ theories where the two fermion species are not related by
$\mathrm{SU}(2)$ rotations but only by a discrete exchange, so that $\mathrm{SU}(2)$ is reduced to $\operatorname{Pin}(2)_{-}$. Below, we often neglect discrete symmetry generators, and we refer to this case as having $\mathrm{U}(1) \times \mathrm{U}(1)$ symmetry [63].

By applying the fermion-fermion duality of a single species of Dirac fermion to each of the two fermion species, Refs. [33,35,37] demonstrated that, similar to the bosonic easy-plane $\mathrm{CP}^{1}$ model, this theory is self-dual, i.e., it is dual to another $N_{f}=2$ QED:

$$
\mathcal{L}_{\mathrm{QED}-\mathrm{dual}}=\sum_{j=1}^{2} i \bar{\chi}_{j} \not_{\tilde{a}} \chi_{j}+\cdots .
$$

Given that a particular basis in flavor space had to be selected to perform this duality, we are, strictly speaking, restricting to theories with just $\mathrm{U}(1) \times \mathrm{U}(1)$ continuous symmetry. The dual theory in Eq. (9) then should also only be taken to have $\mathrm{U}(1) \times \mathrm{U}(1)$ continuous symmetry. However, we later discuss the possibility that with full $\mathrm{SU}(2)$ flavor symmetry this duality survives. As in the easyplane $\mathrm{NCCP}^{1}$ model, the roles of the gauge-flux conservation symmetry and the relative phase rotation symmetry between the two Dirac fermions are exchanged in the dual QED theory. The self-duality is obtained by applying the fermionic particle-vortex duality $[30,31,38,40]$ to both flavors of fermions: $\psi_{1} \rightarrow \chi_{2}, \psi_{2} \rightarrow \chi_{1}$. Since the Dirac mass term is odd under the particle-vortex duality, the selfduality sends $\bar{\psi}_{1} \psi_{1} \rightarrow-\bar{\chi}_{2} \chi_{2}$ and $\bar{\psi}_{2} \psi_{2} \rightarrow-\bar{\chi}_{1} \chi_{1}$.

The IR fate of $\mathrm{QED}_{3}$ at $N_{f}=2$ is controversial at this stage. It is not clear whether at low energy the Dirac fermions will spontaneously break the flavor symmetry and gain a mass of the form $m \bar{\psi} \sigma^{z} \psi-\mathrm{a}$ long-standing issue known as chiral symmetry breaking. Recent numerics [45], however, suggests the possibility that this theory may be stable in the IR (although an earlier study suggests spontaneous chiral symmetry breaking [48]).

We are also interested in the phases and phase transition of this model when a coupling to an extra scalar $\phi$ is allowed. The resulting model has the Lagrangian

$$
\mathcal{L}_{\mathrm{QED}-\mathrm{GN}}=\sum_{j=1}^{2} i \bar{\psi}_{j} D_{a} \psi_{j}+\phi \bar{\psi} \psi+V(\phi) .
$$

Here, we include a potential $V(\phi)=V(-\phi)$ for the scalar field $\phi$ (we suppress its kinetic term for notational simplicity). The theory is time-reversal symmetric if under time reversal $\phi \rightarrow-\phi$. As the potential $V(\phi)$ is tuned, we expect a phase transition between a time-reversal symmetric phase where $\langle\phi\rangle=0$ and a time-reversal broken one where $\langle\phi\rangle \neq 0$. We usually refer to Eq. (10) when tuned to this transition as the $\mathrm{QED}_{3}$-Gross-Neveu model $\left(\mathrm{QED}_{3}\right.$-GN for short).

Interestingly, with some assumptions, Ref. [29] showed that the low-energy behavior of $N_{f}=2 \mathrm{QED}_{3}$ was 
described by the $\mathrm{O}(4)$ sigma model at $\theta=\pi$ discussed in the previous section, again with the proviso that the sigma model needs to extend to strong coupling. This suggests a connection between the bosonic $\mathrm{NCCP}^{1}$ theories and the fermionic $\mathrm{QED}_{3}$ theories. Below, we sharpen this connection through precise duality statements. This also enables us to understand the emergent IR symmetries of these theories at their critical point.

\section{Summary of results}

We now summarize the key results in this paper. We also point out the sections that discuss these statements (and their subtleties) in detail. This section can be viewed as a map of the paper.

(1) Both the easy-plane and the $\mathrm{SU}(2)$-symmetric $\mathrm{NCCP}^{1}$ models are part of a web of dualities.

We begin with the easy-plane model. It turns out that the easy-plane $\mathrm{NCCP}^{1}$ model is dual to fermionic $\mathrm{QED}_{3}$ with $N_{f}=2$ Dirac fermions. This duality was first mentioned in Ref. [35], and is discussed in detail in Sec. III. As discussed in the literature and reviewed earlier in this section, the two theories also possess their own self-dualities. This leads to a web of four theories, mutually dual to each other, as summarized below:

$$
\begin{aligned}
& \left|D_{b+B} z_{1}\right|^{2}+\left|D_{b+B^{\prime}} z_{2}\right|^{2}-\left|z_{1}\right|^{4}-\left|z_{2}\right|^{4}-\frac{1}{2 \pi} b d\left(B+B^{\prime}\right)-\frac{1}{2 \pi} B d B^{\prime}-\frac{1}{2 \pi} B^{\prime} d B^{\prime} \\
\Leftrightarrow & \left|D_{\tilde{b}-B} w_{1}\right|^{2}+\left|D_{\tilde{b}+B^{\prime}} w_{2}\right|^{2}-\left|w_{1}\right|^{4}-\left|w_{2}\right|^{4}-\frac{1}{2 \pi} \tilde{b} d\left(B^{\prime}-B\right)+\frac{1}{2 \pi} B d B^{\prime}-\frac{1}{2 \pi} B^{\prime} d B^{\prime} \\
\Leftrightarrow & \bar{\psi}_{1} i D_{a-B} \psi_{1}+\bar{\psi}_{2} i D_{a+B} \psi_{2}+\frac{1}{2 \pi} a d B^{\prime}+\frac{1}{4 \pi}\left(B d B-B^{\prime} d B^{\prime}\right) \\
\Leftrightarrow & \bar{\chi}_{1} i D_{\tilde{a}-B^{\prime}} \chi_{1}+\bar{\chi}_{2} i D_{\tilde{a}+B^{\prime}} \chi_{2}+\frac{1}{2 \pi} \tilde{a} d B+\frac{1}{4 \pi}\left(B d B-B^{\prime} d B^{\prime}\right) .
\end{aligned}
$$

Here, $b$ and $\tilde{b}$ are ordinary dynamical U(1) gauge fields, $a$ and $\tilde{a}$ are dynamical gauge fields whose charge- 1 fields are fermions (they are formally known as $\operatorname{spin}_{c}$ connections). We also include background $\mathrm{U}(1)$ gauge fields $B$ and $B^{\prime}$. [64] Various background Chern-Simons terms are included to ensure that all the theories have the same response. Despite the profusion of background terms, the dynamical content of the theories in Eq. (11) is simple. In Sec. III we also discuss various possibilities for the IR fates of the theories in the duality web, paying careful attention to symmetries.

There is a similar web of dualities for the SU(2)invariant $\mathrm{NCCP}^{1}$ model, which we discuss in Sec. IV. The structure of this duality web is very similar to that of the easy-plane case: the $\mathrm{SU}(2)$-invariant $\mathrm{NCCP}^{1}$ model is dual to the $\mathrm{QED}_{3}$-Gross-Neveu theory with $N_{f}=2$, and the two theories both admit their own self-dualities. We summarize the mutual dualities of the four theories in the web below:

$$
\begin{aligned}
& \sum_{\alpha=1,2}\left|D_{b} z_{\alpha}\right|^{2}-\left(\left|z_{1}\right|^{2}+\left|z_{2}\right|^{2}\right)^{2} \\
& \Leftrightarrow \sum_{\alpha=1,2}\left|D_{\tilde{b}} w_{\alpha}\right|^{2}-\left(\left|w_{1}\right|^{2}+\left|w_{2}\right|^{2}\right)^{2} \\
& \Leftrightarrow \sum_{j=1,2} \bar{\psi}_{j} i D_{a} \psi_{j}+\phi \sum_{j=1,2} \bar{\psi}_{j} \psi_{j}+V(\phi) \\
& \Leftrightarrow \sum_{j=1,2} \bar{\chi}_{j} i D_{\tilde{a}} \chi_{j}-\phi \sum_{j=1,2} \bar{\chi}_{j} \chi_{j}+V(\phi),
\end{aligned}
$$

where the potential of Ising scalar field $V(\phi)$ is tuned to the critical point.

(2) Understanding the duality web allows some powerful statements about emergent symmetries of possible IR fixed points for the two $\mathrm{NCCP}^{1}$ models, which is one of the main goals of this paper. In the context of deconfined quantum criticality, we show that in both the easy-plane and SU(2)-symmetric cases the emergent symmetry enables rotating the Landau order parameters of the two phases on either side of the transition.

In the easy-plane case, the duality web in its strongest form implies an emergent $\mathrm{O}(4)$ symmetry. The most basic local (gauge-invariant) operators are the order parameters $\left(n_{1}, n_{2}, n_{3}, n_{4}\right)$, which form a vector representation of the $\mathrm{O}(4)$ symmetry. Since $\mathrm{SO}(4) \sim[\mathrm{SU}(2) \times \mathrm{SU}(2)] / \mathbb{Z}_{2}$, the vector operators can be rearranged into $S U(2)$ spinors. The complex doublet

$$
\left(\Phi_{1}, \Phi_{2}\right) \sim(\overbrace{n_{3}+i n_{4}}^{\text {Néel }}, \overbrace{n_{1}+i n_{2}}^{\text {VBS }})
$$

is a fundamental under the first $\mathrm{SU}(2)$, and $\left(\Phi_{1}^{*},-\Phi_{2}\right)$ is a fundamental under the second. The improper $\mathrm{O}(4)$ reflection is represented as complex conjugation on one of the components of $\Phi$. The two complex operators are represented in each theory as 


$$
\begin{aligned}
\left(\Phi_{1}, \Phi_{2}\right) & \sim\left(z_{1}^{\dagger} z_{2}, \mathcal{M}_{b}\right) \\
& \sim\left(\mathcal{M}_{\tilde{b}}^{\dagger}, w_{2}^{\dagger} w_{1}\right) \\
& \sim\left[\psi_{2}^{\dagger} \mathcal{M}_{a},\left(\psi_{1}^{\dagger} \mathcal{M}_{a}\right)^{\dagger}\right] \\
& \sim\left[\left(\chi_{2}^{\dagger} \mathcal{M}_{\tilde{a}}\right)^{\dagger},\left(\chi_{1}^{\dagger} \mathcal{M}_{\tilde{a}}\right)^{\dagger}\right],
\end{aligned}
$$

where $\mathcal{M}_{b}$ is the monopole (instanton) operator for the gauge field $b$. (In QED the monopole configuration induces one zero mode for each Dirac fermion, and gauge invariance requires exactly one of the two zero modes to be filled [65]. The notation $\psi_{1}^{\dagger} \mathcal{M}_{a}$ denotes a monopole in $a$ with the zero mode from the Dirac fermion $\psi_{1}$ filled.) The $\mathrm{O}$ (4) symmetry is discussed in detail in Sec. III. Its implications for numerical simulations are discussed in Sec. VIII.

In the SU(2)-invariant case, the duality web implies an emergent $\mathrm{SO}(5)$ symmetry. The most basic local operators form a vector representation of this $\operatorname{SO}(5):\left(n_{1}, n_{2}, n_{3}, n_{4}, n_{5}\right)$. They are represented in $\mathrm{NCCP}^{1}$ and QED-GN theories as

$$
\begin{aligned}
\left(n_{1}, n_{2}, n_{3}, n_{4}, n_{5}\right) & \sim\left(2 \operatorname{Re} \mathcal{M}_{b}, 2 \operatorname{Im} \mathcal{M}_{b}, z^{\dagger} \sigma_{x} z, z^{\dagger} \sigma_{y} z, z^{\dagger} \sigma_{z} z\right) \\
& \sim\left(w^{\dagger} \sigma_{x} w,-w^{\dagger} \sigma_{y} w, 2 \operatorname{Re} \mathcal{M}_{\tilde{b}},-2 \operatorname{Im} \mathcal{M}_{\tilde{b}}, w^{\dagger} \sigma_{z} w\right) \\
& \sim\left[\operatorname{Re}\left(\psi_{1}^{\dagger} \mathcal{M}_{a}\right),-\operatorname{Im}\left(\psi_{1}^{\dagger} \mathcal{M}_{a}\right), \operatorname{Re}\left(\psi_{2}^{\dagger} \mathcal{M}_{a}\right), \operatorname{Im}\left(\psi_{2}^{\dagger} \mathcal{M}_{a}\right), \phi\right] \\
& \sim\left[\operatorname{Re}\left(\chi_{1}^{\dagger} \mathcal{M}_{\tilde{a}}\right),-\operatorname{Im}\left(\chi_{1}^{\dagger} \mathcal{M}_{\tilde{a}}\right), \operatorname{Re}\left(\chi_{2}^{\dagger} \mathcal{M}_{\tilde{a}}\right),-\operatorname{Im}\left(\chi_{2}^{\dagger} \mathcal{M}_{\tilde{a}}\right), \phi\right] .
\end{aligned}
$$

This $\mathrm{SO}(5)$ symmetry is discussed in detail in Sec. IV. It has been numerically observed in Ref. [15], providing a strong support to our duality web. Its further implications are discussed in Secs. IV E and VIII.

(3) The easy-plane theory has several $\mathbb{Z}_{2}$ (or $\mathbb{Z}_{2}$-like) symmetries which are anomalous. In the context of lattice quantum magnetism these symmetries include the $\mathbb{Z}_{2}$ spin-flip, time-reversal, and lattice translation symmetries (see Sec. III B 2). Under these symmetries the Lagrangian is invariant only up to a background term:

$$
\mathcal{L} \rightarrow \mathcal{L}-\frac{1}{2 \pi} B_{1} d B_{2}
$$

where $B_{1}=B-B^{\prime}$ and $B_{2}=B+B^{\prime}$ are the properly quantized background $\mathrm{U}(1)$ gauge fields. On the lattice this anomaly is harmless since one of the $\mathrm{U}(1)$ symmetries is really a discrete lattice rotation symmetry. However, if one wants to formulate the theory with all these symmetries realized in an on-site manner, the theory can only exist on the surface of a three-dimensional bulk. The symmetry anomaly can be canceled by a bulk mutual $\Theta$ term:

$$
-\frac{1}{4 \pi} \int_{\text {Bulk }} d B_{1} \wedge d B_{2}
$$

All the dualities on the surface are then related to the electric-magnetic dualities in the bulk. Many symmetry actions that appear to be complicated on the surface (in certain pictures) become obvious once the bulk view is taken. We discuss this in Sec. V for the easy-plane theory.

(4) None of the field theories in the duality web, Eq. (12), possess the full $\mathrm{SO}(5)$ symmetry explicitly-the symmetry is at best emergent in the IR. Further, just as in the easy-plane case, the $\mathrm{SO}(5)$ symmetry is anomalously realized. We also discuss two (renormalizable) field theories with explicit $\mathrm{SO}(5)$ symmetry in Sec. VI. The first one is $\mathrm{QCD}_{3}$ with $N_{f}=2$ :

$$
\mathcal{L}=\sum_{v=1,2} i \bar{\psi}_{v} \gamma^{\mu}\left(\partial_{\mu}-i a_{\mu}\right) \psi_{v},
$$

where $a$ is an $\mathrm{SU}(2)$ gauge field and $\psi_{1,2}$ are two $\mathrm{SU}(2)$-fundamental fermions. This theory can be obtained from the square lattice spin-1/2 model through a standard parton construction with a $\pi$-flux mean-field ansatz, and it has an $\mathrm{SO}(5)$ symmetry which becomes manifest when Eq. (18) is written in terms of Majorana fermions. The second theory is a Higgs descendent of $\mathrm{QCD}_{3}$, where the $\mathrm{SU}(2)$ gauge symmetry is Higgsed down to $\mathrm{U}(1)$ :

$\mathcal{L}=\sum_{i=1}^{4} i \bar{\psi}_{i} \gamma^{\mu}\left(\partial_{\mu}-i a_{\mu}\right) \psi_{i}+\left(\lambda \mathcal{M}_{a}+\right.$ H.c. $)$,

where $a_{\mu}$ is now a $\mathrm{U}(1)$ gauge field, and the term $\mathcal{M}_{a}$ represents (schematically) monopole tunneling (instanton) events. In both theories the Dirac fermions transform in the spinor representation of $\mathrm{SO}(5)$. The $\mathrm{SO}(5)$-vector operators are simply the mass operators that are time-reversal invariant.

While the IR fates of the theories Eqs. (18) and (19) are unknown, both theories have the same 
symmetry anomaly as the deconfined critical point. Therefore, one possible scenario, among others, is that one of these theories will flow to the deconfined critical point in the IR.

(5) The full $\mathrm{SO}(5)$ symmetry of the deconfined critical point is anomalous, as revealed already by the sigma model approach. The manifestly SO(5)-invariant QCD theory makes it possible to analyze the anomaly in more detail. We show in Secs. VIC and VII that $\mathrm{QCD}_{3}$ with $N_{f}=2$, with the full $\mathrm{SO}(5) \times Z_{2}^{T}$ symmetry, can only be realized on the surface of a three-dimensional bosonic symmetry-protected topological state. To characterize this SPT state, we can introduce a background $\mathrm{SO}(5)$ gauge bundle $A^{5}$ in the $(3+1) \mathrm{D}$ bulk. The topological response to $A^{5}$ is given by a discrete theta angle [in contrast to the more familiar theta angle in $(3+1)$ D which can be continuously varied]. Section VIC provides a physical derivation of these results which are then rederived by more formal methods in Sec. VII. [66] We show that the partition function of the $(3+1)$ D SPT for a given $\mathrm{SO}(5)$ gauge field configuration is

$$
Z\left[A^{5}\right]=\left|Z\left[A^{5}\right]\right| e^{i \pi \int w_{4}\left[A^{5}\right]},
$$

where $w_{4}\left[A^{5}\right]$ is known as the fourth Stiefel-Whitney class of the $\mathrm{SO}(5)$ gauge bundle. Though the IR fate of $\mathrm{QCD}_{3}$ with $\mathrm{SO}(5) \times Z_{2}^{T}$ symmetry is not known, we show that it must either break this symmetry spontaneously or flow to a CFT. Gapped symmetrypreserving phases (even with topological order) are prohibited.

(6) We also discuss the implications of these dualities (for example, for numerical simulations) extensively in Sec. VIII. We outline a variety of numerical tests of many aspects of the dualities. We also show how numerical calculations on fermionic $\mathrm{QED}_{3}$ and $\mathrm{QED}_{3}$ - GN may provide a new handle on issues associated with deconfined critical points.

(7) In Sec. IX, we discuss what is currently known about the deconfined critical points from simulations. In particular, we discuss the possibility of "pseudocritical" behavior for deconfined critical points [14]. It is possible that the theories discussed in this paper do not flow to stable CFTs, which in the context of deconfined critical point means that the transition is ultimately first order. But the flow to instability could be very slow, giving rise to a very large correlation length, and scaling behavior can still hold up to this very large scale (with exponents drifting as the scale increases). This pseudocritical scenario could potentially reconcile various seemingly conflicting results from numerical simulations: the observance of the emergent $\mathrm{SO}(5)$ symmetry, the drifting of the scaling behavior, and the constraints on exponents of $\mathrm{SO}(5)$-invariant CFTs from conformal bootstrap [25,26]. In this scenario, the dualities and emergent symmetries discussed in this paper can still hold below the (very large) correlation length.

We should point out that the pseudocritical scenario may be more broadly relevant to many quantum phase transitions: in such a scenario the system shows quantum critical behavior above a very low temperature scale $T^{*}$, below which the criticality eventually disappears.

Related field-theoretic work on these dualities has recently appeared in Ref. [67].

\section{EASY-PLANE NCCP ${ }^{1}$ AND FERMIONIC $N_{f}=2 \mathbf{Q E D}_{3}$}

We propose a duality of the easy-plane $\mathrm{NCCP}^{1}$ model to fermionic $N_{f}=2 \mathrm{QED}_{3}$ :

$$
\begin{aligned}
& \left|D_{b+B} z_{1}\right|^{2}+\left|D_{b+B^{\prime}} z_{2}\right|^{2}-\left|z_{1}\right|^{4}-\left|z_{2}\right|^{4}-\frac{1}{2 \pi} b d\left(B+B^{\prime}\right) \\
& -\frac{1}{2 \pi} B d B^{\prime}-\frac{1}{2 \pi} B^{\prime} d B^{\prime} \\
& \Leftrightarrow \bar{\psi}_{1} i D_{a-B} \psi_{1}+\bar{\psi}_{2} i D_{a+B} \psi_{2}+\frac{1}{2 \pi} a d B^{\prime} \\
& +\frac{1}{4 \pi}\left(B d B-B^{\prime} d B^{\prime}\right) .
\end{aligned}
$$

Here, $b$ and $a$ are the dynamical U(1) gauge fields, [68] and we also include background $\mathrm{U}(1)$ gauge fields $B$ and $B^{\prime}$. Various background Chern-Simons terms are included to ensure that the theories on the two sides have the same response (we elaborate on this below). In Appendix A, we present the above duality in a more precise and compact, but less physically intuitive, form. The identification of the Néel and VBS order parameters in the $z$ theory is reviewed in Sec. II A above. We identify these order parameters in the fermionic description in Sec. III A below.

Both theories have been examined in independent numerical studies. This duality implies that two topical issues (fixed points of $\mathrm{QED}_{3}$ at $N_{f}=2$ and the easy-plane $\mathrm{NCCP}^{1}$ ) are closely related. A "weak" form of the duality is the assertion that easy-plane $\mathrm{NCCP}^{1}$ is equivalent to $\mathrm{QED}_{3}$ perturbed by interactions that break the flavor $\mathrm{SU}(2)$ symmetry to $\mathrm{U}(1)$. These perturbations are formally irrelevant at the free UV fixed point of $\mathrm{QED}_{3}$, where the gauge field $a_{\mu}$ is decoupled from the fermions. If these interactions are not important for the IR behavior, as one would naively guess, then the IR fate of easy-plane $\mathrm{NCCP}^{1}$ will be the same as the fate of $\mathrm{QED}_{3}$ with full flavor $\mathrm{SU}(2)$ symmetry, and the IR behavior will have an enlarged emergent symmetry if $\mathrm{QED}_{3}$ flows to a nontrivial fixed point. 
In the deconfined criticality context, the easy-plane models that have been studied show first-order transitions [20,69-72]. However, on the fermionic side, a recent numerical study [45] found evidence for an IR CFT in Eq. (8) with $\mathrm{SU}$ (2) flavor symmetry (in contrast to earlier calculations [48]). Recent numerics on the quantum phase transition between a particular 2D bosonic SPT state and the trivial state, which presumably is described by Eq. (8) with tuning parameter $\sum_{j=1}^{2} m \bar{\psi}_{j} \psi_{j}$, also show evidence of a continuous phase transition [46,47]. [73] In light of the duality it may be interesting to revisit easy-plane deconfined criticality.

\section{A. Justification for the duality}

We first show how the fermionic dual of the easy-plane $\mathrm{NCCP}^{1}$ model may be justified. This duality has already been mentioned in Ref. [35].

First, recall the "basic" boson-fermion duality [34] relating the free Dirac fermion to a Wilson-Fisher boson coupled to $\mathrm{U}(1)_{1}$. There are two closely related versions of this duality (for early works on related dualities see Refs. [74,75]). The Dirac side is (with our conventions)

$$
\mathcal{L}_{f 1}=i \bar{\psi} \not_{A} \psi .
$$

One version of the dual boson theory is

$\mathcal{L}_{b 1}=\left|D_{b} \phi\right|^{2}-|\phi|^{4}+\frac{1}{2 \pi} b d A+\frac{1}{4 \pi} b d b+\frac{1}{8 \pi} A d A$.

The other dual boson theory is

$\mathcal{L}_{b 2}=\left|D_{\hat{b}} \hat{\phi}\right|^{2}-|\hat{\phi}|^{4}-\frac{1}{2 \pi} \hat{b} d A-\frac{1}{4 \pi} \hat{b} d \hat{b}-\frac{1}{8 \pi} A d A$.

The two boson theories are simply related: $\hat{\phi}$ is the vortex dual of $\phi$. Let us also recall the mapping of the relevant "mass" operators. On the Dirac side, a mass term $m \bar{\mu} \psi$ with $m>0$ maps to $r|\phi|^{2}$ with $r>0$ in Eq. (24) while it maps to $-\hat{r}|\hat{\phi}|^{2}$ with $\hat{r}>0$ in Eq. (25). This change of sign of the boson mass between the two bosonic theories is exactly what we expect given that $\hat{\phi}$ is the vortex dual of $\phi$.

Now, starting with the interacting fermionic theory in Eq. (22), we use the dual theory in Eq. (24) for the first flavor of fermion and the dual theory in Eq. (25) for the second flavor. The resulting dual theory, in terms of bosons $\phi_{I}$ and dynamical gauge fields $b_{I}(I=1,2)$, is

$$
\begin{aligned}
\mathcal{L}_{b 12}^{\prime}= & \sum_{I=1,2} \mathcal{L}\left[\phi_{I}, b_{I}\right]+\frac{1}{2 \pi} b_{1} d(a-B)+\frac{1}{4 \pi} b_{1} d b_{1} \\
& +\frac{1}{8 \pi}(a-B) d(a-B)-\frac{1}{2 \pi} b_{2} d(a+B)-\frac{1}{4 \pi} b_{2} d b_{2} \\
& -\frac{1}{8 \pi}(a+B) d(a+B)+\frac{1}{2 \pi} a d B^{\prime} \\
& +\frac{1}{4 \pi}\left(B d B-B^{\prime} d B^{\prime}\right),
\end{aligned}
$$

where $\mathcal{L}\left[\phi_{I}, b_{I}\right]$ contains the kinetic and potential terms for $\phi_{I}$ and $b_{I}$. Integrating out the dynamical gauge field $a$ will impose the following constraint:

$$
b_{1}-b_{2}-B+B^{\prime}=0 \text {. }
$$

This implies that we can define a new dynamical gauge field $b$ such that $b_{1}=b+B$ and $b_{2}=b+B^{\prime}$. Then, Eq. (26) becomes exactly the first line of Eq. (22) (after identifying $\phi$ with $z_{1}$ and $\hat{\phi}$ with $z_{2}$ ).

In addition to the formal derivation above, in the following we perform various consistency checks of the duality and make a few further comments. We defer until Sec. III B a detailed discussion of the matching of symmetries (explicit or emergent) on the two sides, and their implications.

(1) Clearly both sides have (at least) $\mathrm{U}(1) \times \mathrm{U}(1)$ symmetry, probed by the two background gauge fields $B$ and $B^{\prime}$. On the boson side the gaugeinvariant operator $z_{1}^{*} z_{2}$ has charges $q_{B}=+1$, $q_{B^{\prime}}=-1$, and the monopole operator $\mathcal{M}_{b}$ has $q_{B}=q_{B^{\prime}}=+1$. It is actually more natural to define $B_{1}=B-B^{\prime}$ and $B_{2}=B+B^{\prime}$ and to define $\Phi_{B_{1}}$ and $\Phi_{B_{2}}$ as the order parameters charged under the corresponding global U(1)'s. That is, if we let $\left(q_{1}, q_{2}\right)$ denote the charges under $U_{B_{1}}(1)$ and $U_{B_{2}}(1)$, then $\Phi_{B_{1}}$ carries charges $(1,0)$ and $\Phi_{B_{2}}$ carries $(0,1)$. We have

$$
\Phi_{B_{1}}=z_{1}^{*} z_{2}, \quad \Phi_{B_{2}}=\mathcal{M}_{b} .
$$

These are the two Landau order parameters (Néel and VBS, respectively, in the quantum magnet realization), one of which orders on each side of the putative deconfined QCP.

On the fermion side, a monopole operator $\mathcal{M}_{a}$ is associated with two complex fermion zero modes $f_{1,2}$ from the two Dirac fermions, and gauge invariance requires filling one of the zero modes [65]. Therefore, the operators $f_{j}^{\dagger} \mathcal{M}_{a}$ are gaugeinvariant bosons with charges $q_{B}=-1, q_{B^{\prime}}=-1$ (for $j=1$ ) and $q_{B}=+1, q_{B^{\prime}}=-1$ (for $j=2$ ). Clearly they can be identified with the corresponding Landau order parameters on the bosonic side.

The bosonic side of Eq. (22), after a redefinition $b \rightarrow b-B^{\prime}$, can be written as simply

$$
\left|D_{b+B_{1}} z_{1}\right|^{2}+\left|D_{b} z_{2}\right|^{2}-\left|z_{1}\right|^{4}-\left|z_{2}\right|^{4}-\frac{1}{2 \pi} b d B_{2} .
$$

Below, we use both forms of the easy-plane $\mathrm{NCCP}^{1}$ Lagrangian.

(2) The derivation above makes clear that the correspondence for mass operators is 


$$
m_{1} \bar{\psi}_{1} \psi_{1}+m_{2} \bar{\psi}_{2} \psi_{2} \Leftrightarrow m_{1}\left|z_{1}\right|^{2}-m_{2}\left|z_{2}\right|^{2} .
$$

This is because we use the first boson-fermion duality Eq. (24) for the first fermion, and the second boson-fermion duality Eq. (25) for the second fermion. It is easy to check that the phases obtained by adding such mass operators match in the bosonic and fermionic descriptions.

On the bosonic side turning on $r\left(\left|z_{1}\right|^{2}+\left|z_{2}\right|^{2}\right)$ will drive the system into an ordered phase with $\left\langle\Phi_{B_{1}}\right\rangle \neq 0$ or one with $\left\langle\Phi_{B_{2}}\right\rangle \neq 0$ depending on the sign of $r$ [76]. If $r>0$, we have $\left\langle z_{1}\right\rangle,\left\langle z_{2}\right\rangle \neq 0$, a Higgsed gauge field $b_{\mu}$, and a nonzero expectation value $\left\langle z_{1}^{*} z_{2}\right\rangle \neq 0$, which leads to a Meissner effect for $B_{1}$. If $r<0$, we have a free dynamical Maxwell photon at low energy which leads to the Meissner effect for $B_{2}$, via the mutual Chern Simons term in Eq. (29). The mass term corresponds on the fermionic side to $r \bar{\psi} \sigma^{z} \psi$, which gaps out the fermions. Integrating them out produces the term $[1 /(2 \pi)] \operatorname{sgn}(m) a d B$. Together with the $[1 /(2 \pi)] a d B^{\prime}$ term in Eq. (22), this again leads to the Meissner effect of either $B_{1}$ or $B_{2}$ depending on the sign of $m$. This is consistent with the operator identification discussed above.

(3) We can also turn on an antisymmetric mass $\mu\left(\left|z_{1}\right|^{2}-\left|z_{2}\right|^{2}\right)$ on the bosonic side. For $\mu>0$, we have $\left\langle z_{1}\right\rangle \neq 0$ but $\left\langle z_{2}\right\rangle=0$, which gaps out $b$ and sets $b=-B$. So we get a gapped phase with response $[1 /(2 \pi)]\left(B d B-B^{\prime} d B^{\prime}\right)=[1 /(2 \pi)] B_{1} d B_{2}$. If we reinterpret $B$ as a "charge" probe and $B^{\prime}$ as a "spin" probe, this corresponds to the response of the bosonic integer quantum Hall (BIQH) state $[77,78]$. For $\mu<0$, we get a gapped phase with a trivial response. [In the context of deconfined criticality in quantum magnets, where $\mathrm{U}(1)_{B_{2}}$ is only emergent, both of these are trivial phases in which the Néel order parameter is polarized along the $z$ axis.]

On the fermionic side these phases can be reproduced by a symmetric mass term $\mu\left(\bar{\psi}_{1} \psi_{1}+\bar{\psi}_{2} \psi_{2}\right)$ : integrating out the fermions leaves the action

$$
\begin{aligned}
& \frac{1}{4 \pi} \operatorname{sgn}(\mu)(a d a+B d B)+\frac{1}{2 \pi} a d B^{\prime} \\
& +\frac{1}{4 \pi}\left(B d B-B^{\prime} d B^{\prime}\right) .
\end{aligned}
$$

Since the term $\pm[1 /(4 \pi)] a d a$ is a trivial topological field theory, it can be integrated out safely and we produce the same response theory as on the bosonic side. It is also easy to see that no thermal-Hall conductance (i.e., no chiral edge state) is generated for either phase (in either picture), in agreement with known results $[77,78]$ for the trivial insulator and the bosonic integer quantum Hall state.
The responses above indicate that the massless theories of primary interest to us can also be viewed as describing the phase transition between the trivial and bosonic integer quantum Hall insulators. The fermionic picture for this transition was discussed in Refs. $[79,80]$ using parton constructions (see also Appendix B for a different perspective) and more recently in Ref. [81] using a coupled-wire construction. This BIQH transition has been seen numerically, and the results show evidence of a continuous phase transition $[46,47]$.

The mean-field phase diagram implied by the above is summarized in Fig. 1, both in the deconfined criticality context [where $\mathrm{U}(1)_{\text {Néel }}$ is an exact microscopic spin symmetry and $\mathrm{U}(1)_{\mathrm{VBS}}$ is emergent at the critical point] and for the boson integer quantum Hall transition. This mean-field picture captures only the topology of the phase diagram adjacent to the putative critical point: in general, the phase boundaries will meet at a cusp (since $\left|z_{1}\right|^{2}+\left|z_{2}\right|^{2}$ and $\left|z_{1}\right|^{2}-\left|z_{2}\right|^{2}$ will have different scaling dimensions) and will not lie along the axes.

(4) There is potentially a dual formulation of $N=2$ $\mathrm{QED}_{3}$ directly in terms of the bosonic monopoles $f_{j}^{\dagger} \mathcal{M}_{a}$ which are precisely the $\Phi_{B_{1}}, \Phi_{B_{2}}$ defined in Eq. (28). The most we know about this theory if we want to formulate it directly in terms of the physical bosons is that it has the structure of an (anisotropic) $\mathrm{O}$ (4) sigma model at $\theta=\pi$ [29] discussed in

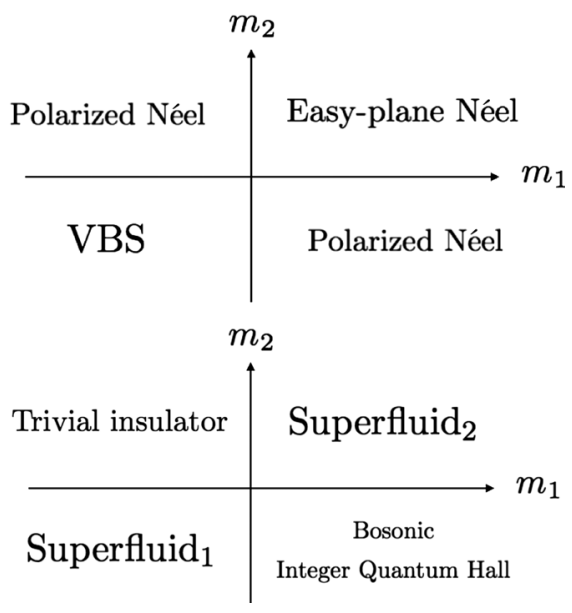

FIG. 1. Mean-field phase diagram for the mass term $m_{1}\left|z_{1}\right|^{2}+$ $m_{2}\left|z_{2}\right|^{2}$ in the easy-plane $\mathrm{NCCP}^{1}$ model. The same phase diagram is obtained from the QED theory with mass term $m_{1} \bar{\psi}_{1} \psi_{1}-m_{2} \bar{\psi}_{2} \psi_{2}$. The upper panel is realized in the context of the quantum magnet, where the $\mathrm{U}(1)_{B_{2}}$ symmetry is only emergent. The lower panel is realized in the context of the integer quantum Hall transition of bosons, where the two superfluid phases correspond to superfluids of the first or second layer, i.e., up or down components of the pseudospin. (Going beyond mean field will move the phase boundaries away from the axes.) 
Sec. II A. This is consistent with the various other known connections between this sigma model and the $\mathrm{QED}_{3}$ theory. This effective field theory is a convenient language for discussing the emergent $\mathrm{O}(4)$ symmetry that would be required if the strong duality conjecture holds for these theories.

\section{B. Symmetries}

We now study the symmetries of the various dual actions, and the implications of the dualities for emergent symmetries of the easy-plane deconfined quantum critical points. Here, we discuss the symmetries from a physical point of view natural in condensed-matter physics. This will make plausible the statements about nontrivial emergent symmetries.

\section{Continuous symmetries}

In this section, we see how the duality web leads to the possibility that the easy-plane $\mathrm{NCCP}^{1}$ theory could have an emergent $\mathrm{O}(4) \times Z_{2}^{T}$ symmetry. [82] In Sec. III C, we express the requirements for this symmetry enhancement more formally in terms of the properties of the putative "mother" $\mathrm{O}(4)$ fixed point. Here, we discuss how the dualities, if they hold in a "strong" form, lead to this emergent symmetry.

In the duality between easy-plane $\mathrm{NCCP}^{1}$ and $\mathrm{QED}_{3}$ we naively expect only that the continuous symmetry of the resulting fermion theory is $\mathrm{U}(1) \times \mathrm{U}(1)$. As explained in Sec. II B, the fermionic action we write down apparently, however, has manifest $[\mathrm{SU}(2) \times \mathrm{U}(1)] / Z_{2}$ symmetry with the $\mathrm{SU}(2)$ corresponding to rotations between the two fermion flavors. Thus, we should allow for terms that break this apparent flavor $\mathrm{SU}(2)$ down to $\mathrm{U}(1)$. The mass term $\bar{\psi} \sigma^{z} \psi$ will accomplish that, but this is precisely the operator whose coefficient is tuned to zero at the transition. The minimal operators with no derivatives that break the flavor SU(2) symmetry are thus four fermion terms, e.g., $\left(\bar{\psi} \sigma^{z} \psi\right)^{2}$. At the free Dirac fermion fixed point these operators are strongly irrelevant. So if the SU(2)-symmetric QED theory has a nontrivial IR fixed point, it is plausible that perturbations breaking $\mathrm{SU}(2)$ to $\mathrm{U}(1)$ are also irrelevant here, and that the theory with microscopic U(1) flavor symmetry flows to this point and has emergent $\mathrm{SU}(2)$ flavor symmetry in addition to the other symmetries already present.

Now consider the self-duality of $\mathrm{QED}_{3}$. In the derivation of Ref. [33] of this self-duality a priori we know only that the $\mathrm{U}(1) \times \mathrm{U}(1)$ symmetry of one side maps to the $\mathrm{U}(1) \times \mathrm{U}(1)$ symmetry of the other side, with the role of the two $U(1)$ symmetries being exchanged by duality (the flavor conservation symmetry on one side becomes the flux conservation symmetry on the other side). Again, it is naively plausible that the $\mathrm{SU}(2)$ flavor symmetry emerges in the infrared on both sides of the duality (see Sec. III C for a more careful discussion). The two flavor SU(2) symmetries on the two sides are distinct from each other, implying that the full continuous symmetry of the $\mathrm{QED}_{3}$ theory is then $[\mathrm{SU}(2) \times \mathrm{SU}(2)] / Z_{2}=\mathrm{SO}(4)$. A version of this argument was previously made in Refs. [37,83]. [84] We will clarify the conditions under which this symmetry enhancement actually happens.

On the fermionic side, the two flavors of Dirac fermions $\psi_{j}$ form a spin-1/2 representation under one of the two $\mathrm{SU}(2)$ subgroups in $\mathrm{SO}(4)$, and the dual Dirac fermions $\chi_{j}$ form a spin-1/2 representation under the other $\mathrm{SU}(2)$ subgroup. These fermions do not transform in a simple way under the whole $\mathrm{SO}(4)$ group, but this is not problematic since they are not gauge invariant. The gaugeinvariant operators $\mathcal{M}_{a}^{\dagger} f_{j}$ transform as a spinor under the flavor SU(2) of the $\psi$ theory. As these are identified with the boson operators $z_{1}^{*} z_{2}$ and $\mathcal{M}_{b}$, it follows that these two operators are a spinor under this $\mathrm{SU}(2)$. Thus, this $\mathrm{SU}(2)$ rotates

$$
\left(\begin{array}{c}
\Phi_{B_{1}}^{*} \\
-\Phi_{B_{2}}
\end{array}\right) \sim\left(\begin{array}{c}
n_{3}-i n_{4} \\
-n_{1}-i n_{2}
\end{array}\right)
$$

as a spinor. It is easy to see that under the flavor $\mathrm{SU}(2)$ of the dual $\mathrm{QED}_{3}$ theory,

$$
\left(\begin{array}{c}
\Phi_{B_{1}} \\
\Phi_{B_{2}}
\end{array}\right) \sim\left(\begin{array}{l}
n_{3}+i n_{4} \\
n_{1}+i n_{2}
\end{array}\right)
$$

is rotated as a spinor. This means that the $\mathrm{SO}(4)$ simply rotates the four real components of $\Phi_{B_{1}}, \Phi_{B_{2}}$, i.e., $\left(n_{1}, n_{2}, n_{3}, n_{4}\right)$, into one another. In the quantum magnetism realization these are precisely the Néel and VBS order parameters.

\section{Discrete symmetries}

Let us now turn to discrete symmetries. We have already mentioned the $Z_{2}$ spin-flip symmetry $\mathcal{S}$. For the quantum magnetism realization in spin-1/2 square lattice magnets, we must also discuss lattice translation, lattice rotation, lattice reflection, and time-reversal symmetries.

$Z_{2}$ spin-flip symmetry.-The $Z_{2}$ spin flip $\mathcal{S}$ corresponds in the microscopic spin model to a rotation of the spin at each site by $\pi$ around the $x$ axis. This is a subgroup of spin $\mathrm{SO}(3)$ symmetry that is presumed to be retained in the easyplane model. In the context of easy-plane deconfined criticality, this symmetry ensures that the only tuning parameter across the transition is the symmetric mass term $r\left(\left|z_{1}\right|^{2}+\left|z_{2}\right|^{2}\right)$.

The full action of $\mathcal{S}$ in the easy-plane $\mathrm{NCCP}^{1}$ theory in the presence of background fields is

$$
z_{1} \leftrightarrow z_{2}, \quad B \leftrightarrow B^{\prime}, \quad b \rightarrow b .
$$


Equivalently, $\mathcal{S}$ takes $B_{1} \leftrightarrow-B_{1}, B_{2} \leftrightarrow B_{2}$. As emphasized in Sec. II A, the corresponding action on the Néel and VBS order parameters is

$$
\mathcal{S}\left(\Phi_{B_{1}}\right)=\Phi_{B_{1}}^{*}, \quad \mathcal{S}\left(\Phi_{B_{2}}\right)=\Phi_{B_{2}} .
$$

Thus, this $Z_{2}$ acts as an improper $\mathrm{O}(4)$ rotation on the vector $\left(n_{1}, n_{2}, n_{3}, n_{4}\right)$ formed from the four real components of these fields.

In $\mathrm{QED}_{3}, \mathcal{S}$ is a transformation between $\psi_{j}$ and its dual fermion $\chi_{j}$. Since the mass terms transform under the fermion self-duality as $\bar{\psi}_{1} \psi_{1} \leftrightarrow-\bar{\chi}_{2} \chi_{2}$, the antisymmetric fermion mass term $\bar{\psi} \sigma^{z} \psi$ is invariant, which is consistent with the operator identification described above.

We should point out that in the continuum field theory this symmetry is actually anomalous. In both the boson and fermion pictures the Lagrangian picks up an extra term under this symmetry operation:

$$
\mathcal{L} \rightarrow \mathcal{L}+\frac{1}{2 \pi}\left(B^{\prime} d B^{\prime}-B d B\right)=\mathcal{L}-\frac{1}{2 \pi} B_{1} d B_{2} .
$$

For deconfined criticality realized in a lattice spin system, this anomaly is harmless because the $\mathrm{U}(1)$ symmetry probed by $B_{2}$ is really a discrete lattice rotation symmetry. However, if the symmetries are on site, this theory can only be regularized on the surface of a three-dimensional bulk. We discuss this in more detail in Sec. V.

Including time reversal, which we discuss below, the full symmetry of the easy-plane $\mathrm{NCCP}^{1}$ fixed point may thus be $\mathrm{O}(4) \times Z_{2}^{T}$. Note that the enlargement of $\mathrm{SO}(4)$ to $\mathrm{O}(4)$ is also expected from the standpoint of the nonlinear sigma model with a theta term, Eq. (7). If $\mathrm{O}(4)$ is broken to $\mathrm{SO}(4)$, the value of $\theta$ can be varied away from $\pi$ : this is plausibly a relevant perturbation.

Bosonic self-duality symmetry.-There is also a $Z_{2}$ subgroup, which we denote $\mathcal{S}_{\psi}$, of the $\mathrm{SU}(2)$ flavor symmetry of the $\mathrm{QED}_{3}$ :

$$
\mathcal{S}_{\psi}: \psi_{1} \leftrightarrow \psi_{2}, \quad B \rightarrow-B, \quad a \rightarrow a, \quad B^{\prime} \rightarrow B^{\prime} .
$$

This $\mathcal{S}_{\psi}$ symmetry is not a microscopic symmetry for the quantum magnet. In $\mathrm{NCCP}^{1}$ it becomes the bosonic selfduality, $z_{\alpha} \rightarrow w_{\alpha}$ :

$$
\Phi_{B_{1}} \leftrightarrow \Phi_{B_{2}}^{*} .
$$

This also shows that the $\mathrm{SU}(2)$ flavor group of the $\mathrm{QED}_{3}$ theory must act in a highly nonlocal fashion in the $\mathrm{NCCP}^{1}$ theory.

On the QED side, imposing $\mathcal{S}_{\psi}$ symmetry forces the mass term to be symmetric, $m \bar{\psi}_{j} \psi_{j}$, which gives a transition between two distinct (SPT) gapped phases. On the bosonic side, this symmetry allows the antisymmetric mass term $\mu\left(\left|z_{1}\right|^{2}-\left|z_{2}\right|^{2}\right)$, which also gives the SPT transition.

Time reversal.-We now specialize to realizations of these deconfined critical points at the Néel-VBS transition in square lattice spin-1/2 magnets. Microscopic symmetries then include-in addition to the spin-rotation and spin-flip $\mathcal{S}$ symmetries-time-reversal and lattice symmetries.

Time reversal $T$ is antiunitary and acts on the $\mathrm{NCCP}^{1}$ fields as

$$
\begin{aligned}
T\left(z_{\alpha}\right) & =\epsilon_{\alpha \beta} z_{\beta}, \\
T(b) & =-b-B-B^{\prime}, \\
T(B) & =B, \quad T\left(B^{\prime}\right)=B^{\prime} .
\end{aligned}
$$

(For the gauge fields we indicate only the time-reversal action on the spatial components; the time component will transform with the opposite sign.) Here, $\epsilon=i \sigma_{y}$ is antisymmetric with $\epsilon_{12}=1$. This is consistent with $\Phi_{B_{1}} \rightarrow-\Phi_{B 1}^{*}$ and $\Phi_{B 2} \rightarrow \Phi_{B 2}^{*}$, as befits the Néel and VBS order parameters, respectively (written in complex form). Note that, as with the $Z_{2}$ spin-flip symmetry, the bosonic Lagrangian is invariant only up to an anomaly:

$$
\mathcal{L} \rightarrow \mathcal{L}+\frac{1}{2 \pi}\left(B^{\prime} d B^{\prime}-B d B\right)=\mathcal{L}-\frac{1}{2 \pi} B_{1} d B_{2} .
$$

On the dual $\mathrm{QED}_{3}$ side, time reversal acts as the product $\mathcal{S}_{\psi} \mathcal{T}$ transformation under which

$$
T(\psi)=\mathcal{S}_{\psi} \gamma_{0} \psi, \quad T(a)=-a .
$$

Note that the $\mathrm{QED}_{3}$ theory also has the same anomaly in Eq. (42).

Translation symmetry.-It suffices to discuss unit lattice translations along one direction, say, the $y$ direction ( $x \rightarrow x, y \rightarrow y+a$, where $a$ is the lattice spacing), which we dub $T_{y}$. The Néel and VBS orders clearly transform as

$$
T_{y}\left(\Phi_{B_{1}}\right)=-\Phi_{B_{1}}, \quad T_{y}\left(\Phi_{B_{2}}\right)=\Phi_{B_{2}}^{*} .
$$

In the $\mathrm{NCCP}^{1}$ theory, this is implemented as

$$
\begin{aligned}
T_{y}\left(z_{\alpha}\right) & =\epsilon_{\alpha \beta} z_{\beta}^{*}, \\
T_{y}(b) & =-b, \\
T_{y}(B) & =-B^{\prime}, \quad T_{y}\left(B^{\prime}\right)=-B .
\end{aligned}
$$

Just like with the $Z_{2}$ spin flip, or time-reversal symmetry, the $\mathrm{NCCP}^{1}$ Lagrangian is invariant under $T_{y}$ only up to an anomaly (shift by $\left.[1 /(2 \pi)] B_{1} d B_{2}\right)$. On the $\mathrm{QED}_{3}$ side, $T_{y}$ takes the fermions $\psi$ to their fermionic duals $\chi$ though the 
detailed transformation is different from that of the $Z_{2}$ spinflip symmetry. Specifically, we have

$$
\psi_{1} \leftrightarrow-\chi_{1}^{\dagger}, \quad \psi_{2} \leftrightarrow \chi_{2}^{\dagger}, \quad a \leftrightarrow-\tilde{a} .
$$

Note that the symmetric mass $\bar{\psi} \psi$ is odd under $T_{y}$, while the antisymmetric mass $\bar{\psi} \sigma^{z} \psi$ is even, exactly as expected from the corresponding operators on the bosonic side.

A simple way to understand the action of $T_{y}$ is as follows. From Eq. (44) we see that the action of $T_{y}$ on the physical order parameters is similar to that of $\mathcal{S}$ if we exchange the Néel and VBS orders. To make this precise, consider the modified translation $\tilde{T}_{y}=U_{B_{1}}(\pi) T_{y}$, which combines translation with a $\pi$ rotation of the easy-plane Néel vector. Then the physical order parameters transform as

$$
\tilde{T}_{y}\left(\Phi_{B_{1}}\right)=\Phi_{B_{1}}, \quad \tilde{T}_{y}\left(\Phi_{B_{2}}\right)=\Phi_{B_{2}}^{*} .
$$

Thus, we see that it is precisely the $\mathcal{S}$ operation performed after a charge-conjugation transformation $\mathcal{C}$ that takes all the fields to their charge conjugate. This identification is nicely consistent with the action of $T_{y}$ in the $\mathrm{QED}_{3}$ theory in Eq. (48). The extra (-) sign in Eq. (48) is simply due to the additional $U_{B_{1}}(\pi)$ in the definition of $\tilde{T}_{y}$.

Rotation and reflection symmetries.-Lattice rotations by $\pi / 2$ about a square lattice site act very simply on the Néel and VBS vectors. We have

$$
R_{\frac{\pi}{2}}\left(\Phi_{B_{1}}\right)=\Phi_{B_{1}}, \quad R_{\frac{\pi}{2}}\left(\Phi_{B_{2}}\right)=i \Phi_{B_{2}} .
$$

This is part of a $\mathrm{U}_{B_{2}}(1)$ rotation whose role in the various dualities we have already discussed. A site-centered lattice reflection $R_{y}$, say, about the $x$ axis $(x \rightarrow x, y \rightarrow-y)$, acts as

$$
R_{y}\left(\Phi_{B_{1}}\right)=\Phi_{B_{1}}, \quad R_{y}\left(\Phi_{B_{2}}\right)=\Phi_{B_{2}}^{*} .
$$

To define its action simply let us denote for any gauge field $A=\left(A_{0}, A_{x}, A_{y}\right)$ the reflected version by $R A=\left(A_{0}, A_{x},-A_{y}\right)$. Then, $R_{y}$ acts in the $z$ formulation as

$$
\begin{aligned}
& R_{y}\left(z_{\alpha}\right)=z_{\alpha}, \quad R_{y}(b)=R b+R B+R B^{\prime}, \\
& R_{y}(B)=-R B^{\prime}, \quad R_{y}\left(B^{\prime}\right)=-R B .
\end{aligned}
$$

It is readily checked that the $\mathrm{NCCP}^{1}$ Lagrangian is invariant under this transformation and there is no anomaly. On the fermion side, $R_{y}$ again involves a duality transformation between $\psi$ and $\chi$ :

$$
\psi_{1} \rightarrow \gamma_{y} \chi_{2}, \quad \psi_{2} \rightarrow \gamma_{y} \chi_{1}, \quad a \rightarrow R \tilde{a} .
$$

\section{Allowed symmetry-breaking terms}

The strong forms of the dualities we discuss here involve the emergence of higher symmetries than are present in the
UV Lagrangians. In order for the dualities to hold in the IR without fine-tuning, the hypothetical higher-symmetry fixed point must exist and must be stable to all perturbations allowed by the symmetry of the UV theory. Here, we clarify these stability requirements. As we discuss in Sec. IX, it is also possible that there is no fixed point with the higher symmetry, but that there is a pseudocritical regime up to a large but finite length scale $\xi$; in this case, the requirements should be interpreted in terms of the effective scaling dimensions in this regime.

We consider perturbations of the hypothetical $\mathrm{O}(4)$ symmetric point relevant to $N_{f}=2 \mathrm{QED}_{3}$ and the easyplane $\mathrm{NCCP}^{1}$ model. Here, we take $\mathrm{QED}_{3}$ to be defined with full $\mathrm{SU}(2)$ flavor symmetry, as done, for instance, in the lattice calculations of Ref. [45]. We see that the conditions for the emergence of $\mathrm{O}(4)$ are more stringent for easy-plane $\mathrm{NCCP}^{1}$ than for QED. Therefore, in principle, it is possible that the self-duality of $\mathrm{QED}_{3}$ could hold, with emergent $\mathrm{O}(4)$, but that easy-plane $\mathrm{NCCP}^{1}$ could fail to flow to this fixed point. (By contrast, the requirements for the emergence of $\mathrm{SO}(5)$ are similar for the bosonic and fermionic theories, as we see in Sec. IV D.)

To begin, the hypothetical fixed point must be stable to $\mathrm{O}(4)$-singlet scalar perturbations. We certainly expect a relevant perturbation that is invariant under $\mathrm{SO}(4)=$ $[\mathrm{SU}(2) \times \mathrm{SU}(2)] / Z_{2}$ but not under improper $\mathrm{O}(4)$ transformations: in the language of the sigma model for the field $\left(n_{1}, n_{2}, n_{3}, n_{4}\right)$ (Secs. II A and III B 1$)$, this corresponds to varying the coefficient of the $\theta$ term away from $\pi$. But this perturbation is harmless as it is forbidden by time reversal, and in the easy-plane $\mathrm{NCCP}^{1}$ model also by the $Z_{2}$ spin-flip symmetry $\mathcal{S}$ (Sec. III B 2).

Apart from the $\mathrm{O}(4)$ vector order parameters $n_{a}$ defined in Eq. (33), it is natural to expect the next leading scalar operators to be those in the two- and four-index symmetric tensor representations of $\mathrm{O}(4)$. We denote these $X_{a b}^{(2)}$ and $X_{a b c d}^{(4)}$. At the level of symmetry,

$$
\begin{aligned}
X_{a b}^{(2)} & \sim n_{a} n_{b}-\delta_{a b} n^{2} / 4, \\
X_{a b c d}^{(4)} & \sim n_{a} n_{b} n_{c} n_{d}-(\cdots),
\end{aligned}
$$

where the subtraction $(\cdots)$ makes the operator traceless. $X^{(2)}$ is certainly relevant.

The two-index symmetric tensor $X_{a b}^{(2)}$ corresponds to the $(1,1)$ representation of $\mathrm{SU}(2) \times \mathrm{SU}(2)$. All components of this operator are therefore forbidden by the explicit $S U(2)$ of QED. In the easy-plane model one component, $\sum_{a=1}^{2} X_{a a}^{(2)}$, is allowed but is precisely the tuning parameter for the Néel-VBS transition: i.e., $\frac{1}{2}\left(\left|\Phi_{B_{2}}\right|^{2}-\left|\Phi_{B_{1}}\right|^{2}\right)$ in terms of the complex Néel and VBS order parameters. The four-index symmetric tensor $X^{(4)}$ is the $(2,2)$ representation of $\mathrm{SU}(2) \times \mathrm{SU}(2)$, so again all components are forbidden by the explicit SU(2) of QED. However, in the 
easy-plane $\mathrm{NCCP}^{1}$ model, the Néel-VBS anisotropy $\sum_{a=1}^{2} \sum_{b=3}^{4} X_{a a b b}^{(4)}$ is allowed, and microscopic models on the square lattice will also allow $\sum_{a=1}^{2} X_{\text {aaaa }}^{(4)}$ [85].

Since the easy-plane model allows an $\mathrm{O}(4)$-breaking perturbation that is forbidden for QED, it is conceivable that the QED self-duality holds, with emergent $\mathrm{O}(4)$, but that the strong duality with easy-plane $\mathrm{NCCP}^{1}$ does not hold. This scenario would apply if there was an $\mathrm{O}(4)$ (pseudo)critical regime in which $X^{(4)}$ was relevant, but the perturbations allowed in the QED theories were irrelevant.

The explicit $\mathrm{SU}(2)$ of QED restricts such perturbations to representations of the form ( 0 , integer). In the sigma model language, the simplest such terms involve two derivatives and four powers of $n$, so are plausibly irrelevant, as argued in Ref. [29]. In other words, if there is an $\mathrm{O}(4)$ fixed point that is stable to $\mathrm{O}(4)$-singlet perturbations, it is very likely that QED flows to it.

From the point of view of the fermionic Lagrangians, both types of perturbation (those allowed in the easy-plane model and those allowed in QED) can be cast as fourfermion terms, giving a plausibility argument for their irrelevance. The breaking of the symmetry of QED to that of the easy-plane model allows a four-fermion operator, as discussed in Sec. III B 1. For the possible emergence of $\mathrm{SU}(2) \times \mathrm{SU}(2)$, consider the following "weak" form of the fermion-fermion duality. We expect that in principle there is an exact UV duality between a cutoff version of QED with $\mathrm{SU}(2)_{B} \times \mathrm{U}(1)_{B^{\prime}}$ symmetry and an effective field theory version of QED (with a highly fine-tuned Lagrangian) in which the explicit continuous symmetry is $\mathrm{U}(1)_{B} \times \mathrm{U}(1)_{B^{\prime}}$. [For convenience, we label the groups by the probe field for the U(1) part.] Since the simplest terms that break $\mathrm{SU}(2)_{B^{\prime}}$ are four-fermion terms in this dual description, it is plausible that $\mathrm{SU}(2)_{B^{\prime}}$ will emerge in the continuum, giving full $\mathrm{SU}(2)_{B} \times \mathrm{SU}(2)_{B^{\prime}}$.

\section{SU(2)-SYMMETRIC NCCP AND FERMIONIC QED Q GN $^{-}$}

We now turn to the deconfined critical point with full $\mathrm{SU}(2)$ symmetry. We propose a duality between the SU(2)symmetric $\mathrm{NCCP}^{1}$ model and the $N_{f}=2 \mathrm{QED}_{3}$ - GN theory,

$$
\begin{gathered}
\sum_{\alpha=1,2}\left|D_{b} z_{\alpha}\right|^{2}-\left(\left|z_{1}\right|^{2}+\left|z_{2}\right|^{2}\right)^{2} \\
\Leftrightarrow \sum_{j=1,2} \bar{\psi}_{j} i D_{a} \psi_{j}+\phi \sum_{j=1,2} \bar{\psi}_{j} \psi_{j}+V(\phi),
\end{gathered}
$$

where $\phi$ is a critical Ising field (real scalar), with the Ising terms $(\partial \phi)^{2}-\phi^{4}$ suppressed for notational convenience. The $\mathrm{QED}_{3}$ - GN model has not been studied numerically as far as we know. The duality suggests the possibility of critical behavior with emergent symmetry.
To justify this duality, we consider the phases of the $\mathrm{QED}_{3}$ - GN Lagrangian in Eq. (57), making use of the results for the pure QED in Sec III. First, the phase with a positive mass term for $\phi$ and $\langle\phi\rangle=0$ is expected to be equivalent to $\mathrm{QED}_{3}$, and dual to the critical easy-plane $\mathrm{NCCP}^{1}$ theory. What does the coupling to $\phi$ mean in the bosonic theory? The mass term $\bar{\psi} \psi$ is identified with $\left(\left|z_{1}\right|^{2}-\left|z_{2}\right|^{2}\right)$ in Eq. (30), so the coupling to the scalar field becomes

$$
\phi\left(\left|z_{1}\right|^{2}-\left|z_{2}\right|^{2}\right) .
$$

Now consider the $\mathrm{QED}_{3}$ - GN theory in the phase where $\langle\phi\rangle \neq 0$, induced by turning on a negative mass term for $\phi$. This gives the symmetric mass term for $\mathrm{QED}_{3}$ and the antisymmetric mass for easy-plane $\mathrm{NCCP}^{1}$, as discussed under Eq. (31). The theory becomes trivially gapped (except for response terms). Finally, what does the phase transition associated with the onset of $\langle\phi\rangle$ of the $\mathrm{QED}_{3}$ - GN model correspond to on the boson side? We propose that it is the critical point of the SU(2)-invariant $\mathrm{NCCP}^{1}$ model.

The discussion of the phase diagram above gives a basic consistency check on this duality. The operator identification goes as follows: the Ising field $\phi$ on the fermionic side is dual to $\left|z_{1}\right|^{2}-\left|z_{2}\right|^{2}$ on the bosonic side. The Ising mass $-\lambda \phi^{2}$ is dual to the anisotropy $\lambda\left|z_{1}\right|^{2}\left|z_{2}\right|^{2}$ on the bosonic side [86]. The phases with $\langle\phi\rangle=0$ and $\langle\phi\rangle \neq 0$ correspond, respectively, to the easy-plane critical theory and to a gapped state with easy-axis Néel order.

To be more precise, the duality with the critical SU(2)invariant $\mathrm{NCCP}^{1}$ model requires an emergent $\mathrm{SO}(5)$ symmetry: the basic assumption underlying the duality is that allowed terms in each theory which break this symmetry are irrelevant. We make this explicit in Sec. IV D. Once again we postpone a detailed discussion of the matching of the symmetries of the two sides and their implications until Sec. IV C.

\section{A. Duality from the sigma model}

We now provide an alternative understanding of the proposed duality, from the standpoint of the nonlinear sigma model description of the $\mathrm{SU}(2)$-invariant $\mathrm{NCCP}^{1}$ model. We propose the equivalence between the $(2+1) \mathrm{D}$ $\mathrm{SO}(5)$ nonlinear sigma model with a WZW term at level 1 (extended to a strong-coupling fixed point) and $N=2$ $\mathrm{QED}_{3}$ deformed with a quartic interaction term to a critical point at $\lambda=\lambda_{c}$ :

$$
\begin{aligned}
\mathcal{L} & =\frac{1}{g}\left(\partial_{\mu} \mathbf{n}\right)^{2}+\frac{2 \pi}{\Omega_{4}} \int_{0}^{1} d u n^{a} \partial_{u} n^{b} \partial_{x} n^{c} \partial_{y} n^{d} \partial_{t} n^{e} \\
\Leftrightarrow \mathcal{L} & =\sum_{j=1}^{2} \bar{\psi}_{j} i D_{a} \psi_{j}+\frac{\lambda}{2}\left(\sum_{j=1}^{2} \bar{\psi}_{j} \psi_{j}\right)^{2} .
\end{aligned}
$$


We also identify a relevant perturbation on both sides of the duality:

$$
u\left(n_{5}\right)^{2} \sim u\left(\sum_{j=1}^{2} \bar{\psi}_{j} \psi_{j}\right)^{2} .
$$

When $u>0$, we expect that $n_{5}$ can be treated as effectively zero in the $\mathrm{SO}(5)$ sigma model, so that this theory reduces to an $\mathrm{O}(4)$ nonlinear sigma model with a $\Theta$ term at $\Theta=\pi$ :

$\mathcal{L}=\frac{1}{g}\left(\partial_{\mu} \mathbf{n}\right)^{2}+\frac{\Theta}{\Omega_{3}} n^{a} \partial_{t} n^{b} \partial_{x} n^{c} \partial_{y} n^{d}, \quad \Theta=\pi$.

This theory has been shown [29], with some assumptions, to be the low-energy effective theory of $N=2 \mathrm{QED}_{3}$. Consistent with this, a positive $u$ in the $\mathrm{QED}_{3}$-Gross-Neveu model will drive the system back to $\mathrm{QED}_{3}$.

When $u<0$, the $\mathrm{SO}(5)$ sigma model develops a nonzero expectation value $\left\langle n_{5}\right\rangle$. This spontaneously breaks the $Z_{2}$ subgroup of the $\mathrm{SO}(5)$. Depending on the sign of $\left\langle n_{5}\right\rangle$, the effective $\mathrm{O}(4)$ sigma model for the remaining components (which we may imagine deriving by integrating out fluctuations in $n_{5}$ ) will flow to either $\Theta=2 \pi$ or $\Theta=0$. The $\mathrm{QED}_{3}$-Gross-Neveu model with $u<0$ spontaneously condenses $\left\langle\sum_{j} \bar{\psi}_{j} \psi_{j}\right\rangle$. This precisely yields the two phases with $\Theta=0$ and $2 \pi$. A condensate of $\left\langle\sum_{j} \bar{\psi}_{j} \psi_{j}\right\rangle$ spontaneously breaks the $Z_{2}$ subgroup of $\mathrm{O}(4)$ [the $Z_{2}$ transformation that exchanges the two $S U(2)$ subgroups], which is also the $Z_{2}$ subgroup of $\operatorname{SO}(5): Z_{2}$ takes $\left(n_{1}, n_{2}, n_{3}, n_{4}, n_{5}\right)$ to $\left(-n_{1},-n_{2},-n_{3}, n_{4},-n_{5}\right)$.

\section{B. Self-dualities}

We show that the easy-plane duality Eq. (22) naturally motivates the duality of the $\mathrm{SU}(2)$-symmetric $\mathrm{NCCP}^{1}$ theory in Eq. (56). Following the same logic, the selfdualities of easy-plane $\mathrm{NCCP}^{1}$ and the $\mathrm{QED}_{3}$ theories also motivate further self-dualities with higher symmetries. For the $\mathrm{NCCP}^{1}$ model, this self-duality reads

$$
\begin{aligned}
& \sum_{\alpha=1,2}\left|D_{b} z_{\alpha}\right|^{2}-\left(\left|z_{1}\right|^{2}+\left|z_{2}\right|^{2}\right)^{2} \\
\Leftrightarrow & \sum_{\alpha=1,2}\left|D_{\tilde{b}} w_{\alpha}\right|^{2}-\left(\left|w_{1}\right|^{2}+\left|w_{2}\right|^{2}\right)^{2} .
\end{aligned}
$$

In the dual theory, the $\mathrm{U}(1)$ phase rotation symmetry of the local operator $w_{1}^{*} w_{2}$ corresponds to the flux conservation of the $b$ gauge field in the original theory; likewise, the flux conservation of $\tilde{b}$ in the dual theory corresponds to the $\mathrm{U}(1)$ phase rotation symmetry of $z_{1}^{*} z_{2}$ in the original theory. One can check the consistency of this duality by turning on an anisotropy term:

$$
\lambda\left|z_{1}\right|^{2}\left|z_{2}\right|^{2} \sim \lambda\left|w_{1}\right|^{2}\left|w_{2}\right|^{2} .
$$

When $\lambda>0$, both theories flow to easy-plane $\mathrm{NCCP}^{1}$, where the self-duality holds [87]. When $\lambda<0$, both theories flow to the easy-axis limit, where the system becomes trivially gapped.

Similarly, for the $\mathrm{QED}_{3}$ - GN model we have the selfduality

$$
\begin{aligned}
& \sum_{j=1,2} \bar{\psi}_{j} i D_{a} \psi_{j}+\phi \sum_{j=1,2} \bar{\psi}_{j} \psi_{j}+V(\phi) \\
\Leftrightarrow & \sum_{j=1,2} \bar{\chi}_{j} i \varnothing_{\tilde{a}} \chi_{j}-\phi \sum_{j=1,2} \bar{\chi}_{j} \chi_{j}+V(\phi) .
\end{aligned}
$$

The switching of the two global U(1) symmetries in the two pictures is similar to that in the self-duality of $\mathrm{NCCP}^{1}$. The consistency of this duality is checked by turning on a mass term for the Ising scalar fields on both sides: when $\langle\phi\rangle \neq 0$, both sides are trivially gapped, and when $\phi$ is gapped, the duality follows from the self-duality of $\mathrm{QED}_{3}$.

\section{Symmetries}

We now study the symmetries of the $\mathrm{NCCP}^{1}$ theory and its duals. We simply assume the correctness of the proposed dualities.

The bosonic side has a manifest $\mathrm{SO}(3) \times \mathrm{O}(2)$ symmetry, where the $z$ bosons are $\mathrm{SO}(3)$ spinors, and the global U(1) symmetry is simply the flux symmetry of the gauge field $b$. The fermionic side also has a (different) manifest $[\mathrm{SU}(2) \times \mathrm{U}(1)] / Z_{2}$ symmetry [more precisely, $\left[\mathrm{SU}(2) \times \operatorname{Pin}(2)_{-}\right] / Z_{2}$ when charge conjugation is accounted for].

How do these symmetries act on physical operators? Collecting the various gauge-invariant order parameters, we have (recall that $\mathcal{M}_{b}$ is the monopole operator in the $\mathrm{NCCP}^{1}$ model)

$$
\begin{aligned}
& \left(2 \operatorname{Re} \mathcal{M}_{b}, 2 \operatorname{Im}\left(\mathcal{M}_{b}\right), z^{\dagger} \sigma_{x} z, z^{\dagger} \sigma_{y} z, z^{\dagger} \sigma_{z} z\right) \\
& \quad \sim\left(n_{1}, n_{2}, n_{3}, n_{4}, n_{5}\right) .
\end{aligned}
$$

The operators $\left(n_{3}, n_{4}, n_{5}\right) \sim\left(z^{\dagger} \sigma_{x} z, z^{\dagger} \sigma_{y} z, z^{\dagger} \sigma_{z} z\right)$ form a fundamental representation of the $\mathrm{SO}(3)$ symmetry in the $\mathrm{NCCP}^{1}$ model, while $\left(n_{1}+i n_{2}, n_{3}-i n_{4}\right) \sim 2\left(\mathcal{M}_{b}, z_{2}^{*} z_{1}\right)$ transforms as a spin-1/2 representation of the flavor $S U(2)$ symmetry of the $\mathrm{QED}_{3}$ theory. It is easy to see that the vector in Eq. (65) then forms a vector representation of an enlarged $\mathrm{SO}(5)$ symmetry.

The $\mathrm{O}(2)$ symmetry of the $\mathrm{NCCP}^{1}$ theory acts as follows: proper rotations act on only $\left(n_{1}, n_{2}\right)$, while improper rotations also multiply $\left(n_{3}, n_{4}, n_{5}\right)$ by a minus sign. [Therefore, the $\mathrm{SO}(3) \times \mathrm{O}(2)$ of $\mathrm{NCCP}^{1}$ is indeed a subgroup of $\mathrm{SO}(5)$.] The $\mathrm{U}(1)$ flux symmetry of the $\mathrm{QED}_{3}$ theory acts as a common phase rotation of both $n_{1}+i n_{2}$ and $n_{3}-i n_{4}$. Finally, the charge-conjugation symmetry of $\mathrm{QED}_{3}$, which reverses the charge under this $\mathrm{U}(1)$, acts as $\left(n_{1}, n_{2}, n_{3}, n_{4}\right) \rightarrow\left(-n_{3},-n_{4}, n_{1}, n_{2}\right)$. 
A different and interesting perspective is provided by taking the proposed strong self-duality of the SU(2)invariant $\mathrm{NCCP}^{1}$ model as our logical starting point. In the original model, the operators $\left(z^{\dagger} \sigma_{x} z, z^{\dagger} \sigma_{y} z, z^{\dagger} \sigma_{z} z\right)$ form a vector under a global $\mathrm{SO}(3)$ symmetry. In the dual model, the operators $\left(w^{\dagger} \sigma_{x} w, w^{\dagger} \sigma_{y} w, z^{\dagger} \sigma_{z} w\right)$ form a vector under another global $\mathrm{SO}(3)$. Since $z^{\dagger} \sigma_{z} z \sim w^{\dagger} \sigma_{z} w$, it is easy to see that the operators $\left(w^{\dagger} \sigma_{x} w, w^{\dagger} \sigma_{y} w, z^{\dagger} \sigma_{x} w, z^{\dagger} \sigma_{y} z, z^{\dagger} \sigma_{z} z\right)$ form a vector under an enlarged $\mathrm{SO}(5)$ symmetry. Thus, the strong self-duality of the $\mathrm{SU}(2)$-invariant $\mathrm{NCCP}^{1}$ model implies the presence of an enlarged $\mathrm{SO}(5)$ symmetry. Conversely, the numerical evidence for the emergence of $\mathrm{SO}(5)$ symmetry at the Néel-VBS transition may be taken as support for the proposed self-duality of the SU(2)invariant $\mathrm{NCCP}^{1}$ model.

Further support comes from considering a deformation of the model to reach the easy-plane model. In the $z$ theory, this can be accomplished by perturbing with the operator $\left(z^{\dagger} \sigma_{z} z\right)^{2}$. In the $w$ theory, the same operator is represented as $\left(w^{\dagger} \sigma_{z} w\right)^{2}$. We expect that this is a relevant perturbation (see Sec. IV D). The resulting flow leads directly to the self-duality of the easy-plane $\mathrm{NCCP}^{1}$ model, which has been independently derived (in its weak form). This is a good consistency check on the self-duality of the SU(2)invariant model.

The action of discrete symmetries is similar to the easyplane case discussed above. Note, however, that the $\mathcal{S}$ symmetry is a subgroup of the $\mathrm{SU}(2)$ flavor symmetry of the $\mathrm{NCCP}^{1}$ theory, and that $\mathcal{S}_{\psi}$ is a subgroup of the $\mathrm{SU}(2)$ flavor symmetry of $\mathrm{QED}_{3}$. We, thus, do not need to consider them separately. For square lattice spin- $1 / 2$ magnets, the action of time-reversal and lattice symmetries may be readily inferred from the easy-plane case once we recognize that the extra field $\phi$ transforms identically to $z^{\dagger} \sigma_{z} z$.

\section{Allowed symmetry-breaking terms}

We now study the $\mathrm{SO}(5)$-breaking operators that are allowed by microscopic symmetries. These operators must be irrelevant in order for the dualities and the emergent symmetries to hold in the IR without fine-tuning. Again, we point out that in the situation where there is no fixed point with the higher symmetry, there could still be a pseudocritical regime up to a large but finite length scale $\xi$ (see Sec. IX); in this case, the requirements should be interpreted in terms of the effective scaling dimensions in this regime.

The putative emergent symmetry for the $\mathrm{NCCP}^{1}$ model and QED-Gross-Neveu model is $\mathrm{SO}(5)$. A natural guess is that the leading scalar operators, apart from the $\mathrm{SO}(5)$ vector $n_{a}$ defined in Eq. (65), are those in the two- and fourindex symmetric tensor representations of $\mathrm{SO}(5)$. We denote these $X_{a b}^{(2)}$ and $X_{a b c d}^{(4)}$. At the level of symmetry,

$$
\begin{aligned}
X_{a b}^{(2)} & \sim n_{a} n_{b}-\delta_{a b} n^{2} / 5, \\
X_{a b c d}^{(4)} & \sim n_{a} n_{b} n_{c} n_{d}-(\cdots),
\end{aligned}
$$

where the subtraction $(\cdots)$ makes the operator traceless. $X^{(2)}$ is certainly relevant [88]. The microscopic symmetries of $\mathrm{NCCP}^{1}$ allow the perturbation $\sum_{a=3}^{5} X_{a a}^{(2)}$, which is an anisotropy between Néel and VBS $\left(\sim \frac{2}{5}\left[n_{3}^{2}+n_{4}^{2}+n_{5}^{2}\right]-\right.$ $\left.\frac{3}{5}\left[n_{1}^{2}+n_{2}^{2}\right]\right)$, and QED-GN allows $X_{55}^{(2)}$, corresponding to the mass term for the scalar field $\phi$. Since these are the perturbations that are tuned away to reach the critical point, they do not pose a problem for stability. However, stability does require the irrelevance of $X^{(4)}$, as this gives rise to further symmetry-allowed perturbations. The symmetries of $\mathrm{NCCP}^{1}$ allow the higher Néel-VBS anisotropy $\sum_{a=1}^{2} \sum_{b=3}^{5} X_{a a b b}^{(4)}$. For a quantum antiferromagnet on the square lattice, [89] the anisotropy $\sum_{a=1}^{2} X_{\text {aaaa }}^{(4)}$, which breaks the U(1) symmetry for the VBS down to $Z_{4}$, is also allowed. In QED-GN the anisotropy $X_{5555}^{(4)}$ is allowed.

Stability also requires the irrelevance of all $\mathrm{SO}(5)$-singlet scalar operators. As we discuss in Sec. IX, this requirement is in tension with conformal bootstrap results $[25,26]$. However, the numerical evidence for $\mathrm{SO}(5)$ suggests that there is at least a pseudocritical regime where allowed $\mathrm{SO}(5)$-breaking perturbations, including $X^{(4)}$, are effectively irrelevant.

\section{E. Phase diagram}

We now discuss the phase diagram of the quantum magnet near the SU(2)-invariant deconfined critical point, allowing for a perturbation that breaks the spin symmetry to easy plane. We assume the emergence of $\mathrm{SO}(5)$ symmetry at the $S U(2)$ critical point.

It is useful to organize perturbations into representations of the $\mathrm{SO}(5)$ symmetry. The two perturbations that we must consider live in the symmetric tensor representation $X^{(2)}$ of $\mathrm{SO}(5)$ discussed above (we drop the superscript),

$$
X_{a b} \sim n_{a} n_{b}-\frac{\delta_{a b}}{5} n^{2},
$$

and we denote them

$$
\mathcal{O}_{1}=X_{11}+X_{22}, \quad \mathcal{O}_{2}=X_{55} .
$$

First, the leading perturbation allowed in an SU(2)symmetric spin model is $\delta \mathcal{L}=\lambda_{1} \mathcal{O}_{1}$, which drives the system into the Néel ordered phase for $\lambda_{1}<0$ and into the VBS phase for $\lambda_{1}>0$. Second, breaking the spin symmetry down to that of the easy-plane model allows the anisotropy $\delta \mathcal{L}=\lambda_{2} \mathcal{O}_{2}$. Again, we already know the effect of this operator on its own: it drives the system into an easy-axisordered gapped phase for $\lambda_{2}>0$, and to the Néel-VBS 
phase transition of the easy-plane model, potentially with $\mathrm{O}(4)$ symmetry, for $\lambda_{2}<0$.

The full phase diagram for small $\left(\lambda_{1}, \lambda_{2}\right)$ follows by $\mathrm{SO}(5)$ symmetry, assuming that the only ordered phases in the vicinity of the $\mathrm{SU}(2)$ critical point are those mentioned above. Essentially, each of the three ordered phases is determined by which components of $n_{a}$ are favored by the potential $\lambda_{1}\left(X_{11}+X_{22}\right)+\lambda_{2} X_{55}$. More formally, the transition line $\lambda_{1}=\lambda_{2}>0$ between the Ising and VBS ordered phases may be fixed by noting that it corresponds to the perturbation

$$
\lambda_{1}\left(X_{11}+X_{22}+X_{55}\right)=-\lambda_{1}\left(X_{33}+X_{44}\right),
$$

where we use the tracelessness of $X$. This is related to the Néel ordered line $\lambda_{1}<0, \lambda_{2}=0$ by the $\mathrm{SO}(5)$ rotation $n_{1} \leftrightarrow n_{3}, n_{2} \leftrightarrow n_{4}$, which is precisely the self-duality of the $\mathrm{NCCP}^{1}$ theory. The phase diagram in the $\left(\lambda_{1}, \lambda_{2}\right)$ basis is shown in Fig. 2.

If we neglect perturbations that are irrelevant at the $\mathrm{SO}$ (5) fixed point, the transition between VBS and easyaxis order is governed by a Lagrangian with an emergent $\mathrm{O}(3)$ symmetry rotating $\left(n_{1}, n_{2}, n_{5}\right)$. This is spontaneously broken to $\mathrm{SO}(2)$ once $\lambda_{1}=\lambda_{2}$ flows to an order 1 value, yielding a pair of Goldstone modes. In reality, these Goldstone modes are only approximate if the bare $\lambda_{1}=\lambda_{2}$ is finite; the emergent $\mathrm{O}(3)$ is explicitly broken by dangerously irrelevant higher anisotropies [90] which are allowed by the symmetries of the lattice model. However, these anisotropies will appear only at a parametrically large length scale when the bare $\lambda_{1}=\lambda_{2}$ is small.

Similarly, the line $\lambda_{1}=0, \lambda_{2}<0$, which leads to the easy-plane deconfined transition, has an emergent $\mathrm{O}(4)$ symmetry when higher anisotropies are neglected. Here, however, it is possible that the $\mathrm{O}(4)$ symmetry survives to asymptotically long length scales: this depends on the ultimate fate of the easy-plane theory.

The structure of the phase diagram above could be tested numerically. The most basic test is that the phase boundaries all meet at nonzero angles, showing that the distinct components of $X$ have the same scaling dimension [91]. There is also universal information in the slopes of the

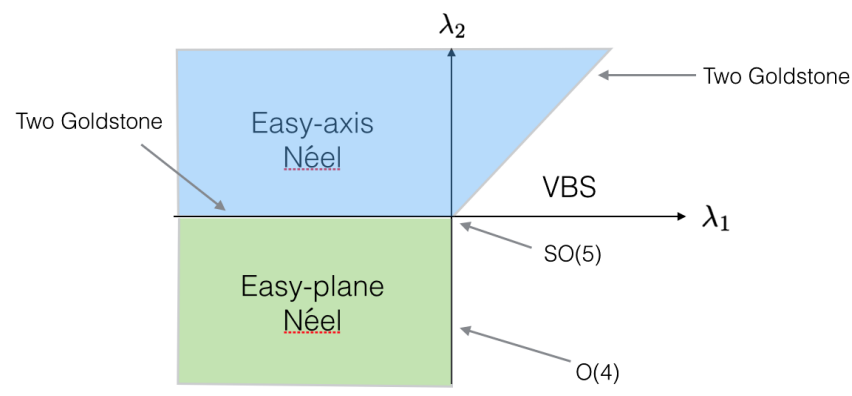

FIG. 2. Phase diagram near the $\mathrm{SO}(5)$-invariant fixed point with perturbation of the form $\lambda_{1}\left(X_{11}+X_{22}\right)+\lambda_{2} X_{55}$. phase boundaries. In the microscopic model a more natural basis for perturbations is $\delta \mathcal{L}=\tilde{\lambda}_{1} \mathcal{O}_{1}+\tilde{\lambda}_{2} \tilde{\mathcal{O}}_{2}$, where $\mathcal{O}_{1}$ is the lattice operator that drives the Néel-VBS transition and $\tilde{\mathcal{O}}_{2} \sim X_{55}+\frac{1}{3}\left(X_{11}+X_{22}\right)$ is a modified easy-plane anisotropy. The numerical coefficient in the latter is fixed by demanding that it belongs to the traceless symmetric tensor representation of spin $\mathrm{SO}(3): \mathcal{A}_{i j} \sim$ $X_{i j}+\frac{1}{3}\left(X_{11}+X_{22}\right)$, where $i, j=3,4,5$. In $\mathrm{NCCP}^{1}, \mathcal{O}_{1} \sim$ $-|z|^{2}$ and $\tilde{\mathcal{O}}_{2} \sim\left[\left|z_{1}\right|^{2}-\left|z_{2}\right|^{2}\right]^{2}-\frac{1}{3}\left[\left|z_{1}\right|^{2}+\left|z_{2}\right|^{2}\right]^{2}$. When we draw the phase diagram in the $\left(\tilde{\lambda}_{1}, \tilde{\lambda}_{2}\right)$ plane, the easy-axis Néel-VBS transition line is at $\tilde{\lambda}_{1}=2 c \tilde{\lambda}_{2}$, and the easyplane Néel-VBS transition line is at $\tilde{\lambda}_{1}=-c \tilde{\lambda}_{2}$. The constant $c>0$ is arbitrary since the normalization of the lattice operators is arbitrary, but the ratio of the slopes of the two lines is a fixed constant which could be checked numerically. The phase diagram in this $\left(\tilde{\lambda}_{1}, \tilde{\lambda}_{2}\right)$ basis is shown schematically in Fig. 3.

Alternately, we may check universal amplitudes using correlation functions, once the location of one of the nontrivial transition lines is determined. Let us normalize $X_{a b}$ so that

$\left\langle X_{a b}(x) X_{c d}(0)\right\rangle=\frac{1}{2}\left(\delta_{a c} \delta_{b d}+\delta_{a d} \delta_{b c}-\frac{2}{5} \delta_{a b} \delta_{c d}\right) \frac{1}{x^{2 \Delta_{2}}}$,

where $\Delta_{2}$ is the scaling dimension of $X_{a b}$. Assume that we can identify (numerically) either the perturbation $\mathcal{O}_{2} \sim-X_{55}$, which drives the system along the VBSeasy-plane Néel phase boundary, or the perturbation $\mathcal{O}_{3} \sim$ $X_{11}+X_{22}+X_{55}$ that drives the system to the first-order transition between the VBS and the easy-axis Néel state. Then by Eq. (70) the following statements, independent of normalization, should be true:

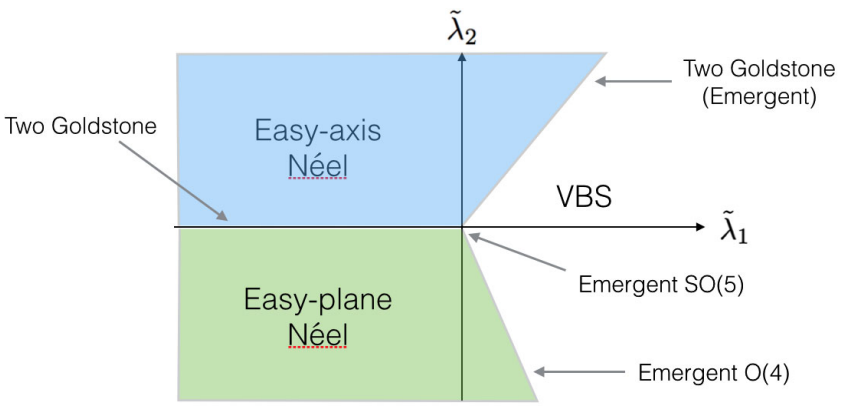

FIG. 3. Phase diagram near the $\mathrm{SO}(5)$-invariant fixed point with perturbation of the form $-\tilde{\lambda}_{1}\left(\left|z_{1}\right|^{2}+\left|z_{2}\right|^{2}\right)+\tilde{\lambda}_{2}\left[\left(\left|z_{1}\right|^{2}-\left|z_{2}\right|^{2}\right)^{2}-\right.$ $\left.\frac{1}{3}\left(\left|z_{1}\right|^{2}+\left|z_{2}\right|^{2}\right)^{2}\right]$. This is the natural perturbation to consider in the context of deconfined criticality in quantum magnets. The emergent $\mathrm{SO}(5)$ symmetry requires that the slope of the lower transition line is twice that of the upper transition line. 


$$
\begin{gathered}
\frac{\left\langle\mathcal{O}_{1}(x) \mathcal{O}_{2}(0)\right\rangle^{2}}{\left\langle\mathcal{O}_{1}(x) \mathcal{O}_{1}(0)\right\rangle\left\langle\mathcal{O}_{2}(x) \mathcal{O}_{2}(0)\right\rangle}=\frac{1}{6}, \\
\frac{\left\langle\mathcal{O}_{1}(x) \mathcal{O}_{3}(0)\right\rangle^{2}}{\left\langle\mathcal{O}_{1}(x) \mathcal{O}_{1}(0)\right\rangle\left\langle\mathcal{O}_{3}(x) \mathcal{O}_{3}(0)\right\rangle}=\frac{4}{9}
\end{gathered}
$$

Similar tests are possible in the QED-Gross-Neveu theory, if the fixed point is found. There, $\mathcal{O}_{2} \sim \phi^{2}$ is the Ising mass operator that drives the system through the Gross-Neveu transition between $\mathrm{QED}_{3}$ and the gapped phase in which $\phi$ has condensed. The fermion chiral mass $\bar{\psi}_{1} \psi_{1}-\bar{\psi}_{2} \psi_{2}$ is a mixture of $\mathcal{O}_{1}$ and $\mathcal{O}_{2}$. The $\mathrm{SU}(2)$ flavor symmetry of QED requires the chiral mass to be orthogonal to $\mathcal{O}_{2}$, so by Eq. (70)

$$
\bar{\psi}_{1} \psi_{1}-\bar{\psi}_{2} \psi_{2} \sim \mathcal{O}_{1}+\frac{1}{2} \mathcal{O}_{2} .
$$

Now if we consider a perturbation of the form $m_{\phi} \phi^{2}+m_{\psi}\left(\bar{\psi}_{1} \psi_{1}-\bar{\psi}_{2} \psi_{2}\right)$, the phase diagram will look like Fig. 4. The phase diagram is symmetric under the reflection across the $m_{\phi}$ axis simply because of the fermion flavor symmetry. The two transition lines near the gapped phase are given by $m_{\phi}= \pm\left[1 /\left(2 c^{\prime}\right)\right] m_{\psi}>0$, with $c^{\prime}>0$ being a normalization-dependent constant. So $c^{\prime}$ alone does not provide nontrivial information. However, it enters into the ratio of correlation functions:

$\frac{\left\langle\phi^{2}(x) \phi^{2}(0)\right\rangle}{\left\langle\left(\bar{\psi}_{1} \psi_{1}-\bar{\psi}_{2} \psi_{2}\right)(x)\left(\bar{\psi}_{1} \psi_{1}-\bar{\psi}_{2} \psi_{2}\right)(0)\right\rangle}=\frac{4}{5}\left(c^{\prime}\right)^{2}$,

which is, in principle, testable.

We end this section with a discussion on the nature of the transitions at $m_{\phi}= \pm\left[1 /\left(2 c^{\prime}\right)\right] m_{\psi}>0$ in Fig. 4. As discussed already, we expect the two transitions to be direct first order, instead of broadening into coexistence phases. How do we understand this from a fermion mean-field point of view? We can calculate the mean-field free energy with respect to $m_{\psi}$ and $\langle\phi\rangle$, treating the fermions as almost

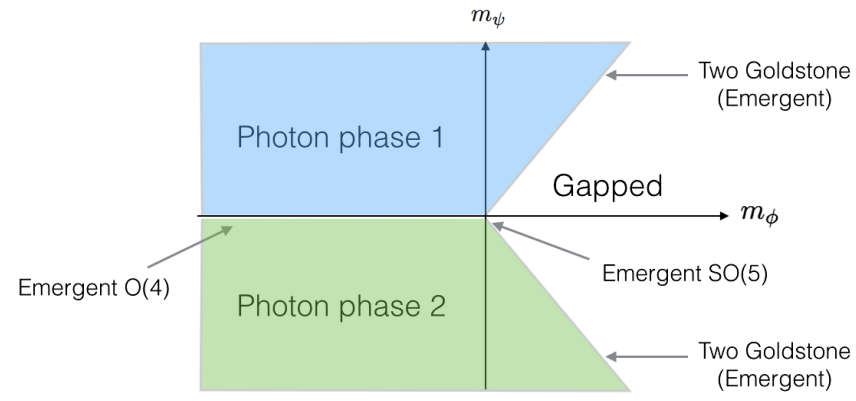

FIG. 4. Phase diagram near the $\mathrm{SO}(5)$-invariant fixed point with perturbation of the form $m_{\phi} \phi^{2}+m_{\psi}\left(\bar{\psi}_{1} \psi_{1}-\bar{\psi}_{2} \psi_{2}\right)$. This is the natural perturbation to consider in the context of $\mathrm{QED}_{3}$-GrossNeveu theory. The emergent $\mathrm{SO}(5)$ symmetry predicts that the slope of the transition lines is related to the relative amplitude of the correlation functions of the two operators, Eq. (73) noninteracting, which is valid when the fermion flavor number $N_{f} \rightarrow \infty$. The result is proportional to

$$
E \sim\left|m_{\psi}+\langle\phi\rangle\right|^{3}+\left|m_{\psi}-\langle\phi\rangle\right|^{3} .
$$

Interestingly, this function gives no preference to either scenario (first order or coexistence). Presumably, fluctuations beyond mean field will break this degeneracy and lead to a direct transition.

\section{BULK INTERPRETATION: $\mathrm{U}(1) \times \mathbf{U}(1)$ THEORY}

From the sigma model point of view, it is known that the symmetries of the field theories discussed so far have to be anomalous [58,92]. Deconfined criticality can nevertheless be realized in quantum magnets because lattice rotation symmetries are not on site, and therefore can be implemented in a seemingly anomalous fashion in the continuum theory. If we want all the symmetries to be on site, the theories must be regularized on the boundary of a $(3+1) \mathrm{D}$ bulk. In this section, we discuss the bulk physics corresponding to the easy-plane deconfined critical point. This provides considerable insight into the duality web and the unconventional symmetry actions of the theory.

In Eq. (36), we see that the spin-flip $\mathcal{S}$, time-reversal, and other symmetries are anomalous:

$$
\mathcal{L} \rightarrow \mathcal{L}-\frac{1}{2 \pi} B_{1} d B_{2}
$$

We initially focus on the $\mathcal{S}$ symmetry. This anomaly is natural from the sigma model approach: the $\mathcal{S}$ symmetry is an improper $\mathrm{O}(4)$ rotation $\left(n_{1}, n_{2}, n_{3}, n_{4}\right) \rightarrow$ $\left(n_{1},-n_{2}, n_{3}, n_{4}\right)$, which fixes $\theta=\pi$, and such a symmetry is typically expected to be anomalous.

The anomaly can be cured by placing the $(2+1) \mathrm{D}$ theory at the boundary of a $(3+1)$ D bosonic SPT insulator with $\left[\mathrm{U}(1) \rtimes Z_{2}\right] \times \mathrm{U}(1)$ symmetry. Let us couple $(3+$ 1)D background gauge fields $B_{1}$ and $B_{2}$ to the two U(1) symmetries such that under $\mathcal{S}$ they transform as

$$
\mathcal{S}: B_{1} \rightarrow-B_{1}, \quad B_{2} \rightarrow B_{2} .
$$

A nontrivial SPT phase of such a bosonic system then has a response characterized by a mutual $\Theta$ term at $\Theta=\pi$ for the two gauge fields $B_{1}$ and $B_{2}$ of the form

$$
-\frac{\Theta}{(2 \pi)^{2}} \int_{\text {Bulk }} d B_{1} \wedge d B_{2}, \quad \Theta=\pi .
$$

Notice that under $\mathcal{S}, \Theta \rightarrow-\Theta$, and therefore $\Theta=\pi$ is protected [93] by the $\mathcal{S}$ symmetry.

Now consider the surface of this boson SPT phase. The bulk $\Theta$ term leads to a surface state with anomalous symmetry realization. Clearly, this anomaly is exactly 
the same as in the $(2+1) \mathrm{D}$ easy-plane $\mathrm{NCCP}^{1}$ field theory, Eq. (36). Specifically, if we add a bulk contribution, $-[1 /(4 \pi)] B_{1} d B_{2}$, to the Lagrangian, defining

$$
\mathcal{L}_{z, \mathrm{SPT}}=\mathcal{L}_{z}-\frac{1}{4 \pi} B_{1} d B_{2}
$$

[where the extra term, which is not well defined as a mutual Chern-Simons term in pure $(2+1) \mathrm{D}$, is really a shorthand notation for the bulk mutual $\Theta$ term at $\Theta=\pi$ ], it is easy to check that $\mathcal{L}_{z, \mathrm{SPT}}$ is indeed invariant under the spin-flip symmetry.

Now imagine gauging the $\mathrm{U}(1)_{B_{1}} \times \mathrm{U}(1)_{B_{2}}$ symmetry in the bulk. An important observation is that, due to the mutual $\Theta$ term, the monopole of one species carries charge $\pm 1 / 2$ of the other species. Let us label the charge-monopole lattice by $\left(q_{e 1}, q_{e 2} ; q_{m 1}, q_{m 2}\right)$. Here, $\left(q_{e 1}, q_{m 1}\right)$ are the electric and magnetic charges under $\mathrm{U}(1)_{B_{1}}$, and so on. The mutual $\Theta$ term implies the relations

$q_{e 2}=\frac{q_{m 1}}{2} \quad(\bmod Z), \quad q_{e 1}=\frac{q_{m 2}}{2} \quad(\bmod Z)$.

Note that $q_{m 1}, q_{m 2} \in Z$.

There is a correspondence between fields in the boundary theory and particles in the bulk theory: bulk electric charges correspond to electrically charged surface fields, and bulk magnetic charges correspond to vortices on the surface. For the "physical" bosons the correspondence is $\Phi_{B_{1}} \sim(1,0 ; 0,0)$ and $\Phi_{B_{2}} \sim(0,1 ; 0,0)$. The surface fields $z_{1,2}$ are vortices in $\Phi_{B_{2}}$, and they carry charge $q_{B_{1}}= \pm 1 / 2$. Their bulk avatars are thus the dyons $z_{1,2} \sim\left( \pm \frac{1}{2}, 0 ; 0,1\right)$.

The bosonic self-duality of the easy-plane $\mathrm{NCCP}^{1}$ theory leads to a description in terms of complex fields $w_{1,2}$, which are vortices of $\Phi_{B_{1}}$ and carry charges $q_{B_{2}}= \pm 1 / 2$. Clearly, their bulk avatars are $w_{1,2} \sim\left(0, \pm \frac{1}{2} ; 1,0\right)$. The surface selfduality is thus connected to the obvious bulk duality between descriptions in terms of these two sets of dyons.

Consider the bound states of these two kinds of dyons with quantum numbers

$$
\left(\frac{1}{2}, \frac{1}{2} ; 1,-1\right), \quad\left(-\frac{1}{2},-\frac{1}{2} ; 1,-1\right) .
$$

These are both fermions. We identify them as the bulk avatars of $\psi_{1,2}$. This can be confirmed directly from the surface theory. Consider the $\mathrm{QED}_{3}$ theory with $\psi_{1,2}$ fermions in Eq. (22) with the added "bulk" contribution $-[1 /(4 \pi)] B_{1} d B_{2}$. Notice that $B=\left(B_{1}+B_{2}\right) / 2$. We see that the $\psi_{1,2}$ fermions indeed have the right charges and vorticities to correspond to these bulk fermionic dyons.

Thus, the duality between the easy-plane $\mathrm{NCCP}_{1}$ theory and the $\mathrm{QED}_{3}$ theory can be understood in terms of a bulk duality that trades the bosonic $\left( \pm \frac{1}{2}, 0 ; 0,1\right)$ particles with the fermionic particles of Eq. (80).
What about the dual fermions $\chi_{1,2}$ ? They correspond to

$$
\left(\frac{1}{2},-\frac{1}{2} ; 1,1\right), \quad\left(-\frac{1}{2}, \frac{1}{2} ; 1,1\right) .
$$

Indeed, this is exactly what is implied by the dual fermionic surface theory.

The fermion-fermion duality of $\mathrm{QED}_{3}$ can thus be related to a corresponding bulk fermion-fermion duality of the $\mathrm{U}(1) \times \mathrm{U}(1)$ gauge theory.

Notice that $\left(q_{e 1}, q_{e 2} ; q_{m 1}, q_{m 2}\right) \rightarrow\left(-q_{e 1}, q_{e 2} ;-q_{m 1}, q_{m 2}\right)$ under $\mathcal{S}$. It is immediately clear that the two fermionic dyons corresponding to $\psi_{1,2}$ become the two dyons corresponding to $\chi_{1,2}$ under $\mathcal{S}$. This offers a bulk interpretation of the nontrivial action of $\mathcal{S}$ on the surface $\mathrm{QED}_{3}$ theory, which exchanges $\psi_{1,2}$ and their dual fermions $\chi_{1,2}$.

Likewise, under the fermion flavor exchange symmetry $\mathcal{S}_{\psi}$, which acts as $B_{1} \leftrightarrow-B_{2}$, the dyons corresponding to $z_{1,2}$ and $w_{1,2}$ are exchanged. This is a simple bulk picture of the symmetry action in the boundary $\mathrm{NCCP}^{1}$ model, which is implemented through the self-duality transform.

Note that the $\mathrm{U}(1) \times \mathrm{U}(1)$ gauge theory has an $\mathrm{Sp}(4, Z)$ invariance corresponding to basis changes in the fourdimensional charge-monopole lattice. This is because the basis change must preserve the area of the unit cell of each two-dimensional subspace corresponding to each of the two U(1) gauge theories. The surface web of dualities we discuss herein can be understood as the effects of various $\operatorname{Sp}(4, Z)$ transformations of the bulk gauge theory.

We should also emphasize that the bulk duality offers a simple picture of the surface duality, but does not prove the surface duality between IR fixed point theories.

Now let us turn briefly to time reversal, which acts on $B_{1,2}$ as

$$
T\left(B_{1,2}\right)=B_{1,2} .
$$

Under this the bulk $\Theta$ term is odd, but as before, $\Theta=\pi$ is time-reversal symmetric. Correspondingly, when a surface is present, the contribution from this bulk $\Theta$ term will exactly cancel the time-reversal anomaly of the surface theories. The bulk charges transform under time reversal as

$$
T:\left(q_{e 1}, q_{e 2} ; q_{m 1}, q_{m 2}\right) \rightarrow\left(-q_{e 1},-q_{e 2} ; q_{m 1}, q_{m 2}\right) .
$$

It is readily checked that this is precisely consistent with the time-reversal action on each of the surface theories.

We already saw that the translation $\tilde{T}_{y}$ can be related to a combination of $\mathcal{S}$ and $\mathcal{S}_{\psi}$ and therefore does not need separate discussion.

\section{THEORIES WITH MANIFEST SO(5) SYMMETRY}

Thus far, none of our field-theoretic descriptions of the deconfined critical point have possessed explicit $\mathrm{SO}(5)$ 
symmetry in the UV: this symmetry, at best, emerges in the IR. An exception is the $\mathrm{SO}(5)$ non-linear sigma model (NLSM) with a WZW term at level 1; however, this model is nonrenormalizable, so while one can infer symmetry information from it, strictly speaking its dynamics in the disordered phase is not well defined. Here, we present two renormalizable theories with explicit $\mathrm{SO}(5)$ symmetry, namely, $N_{f}=2 \mathrm{QCD}_{3}$ and its Higgs descendent $N_{f}=4$ compact $\mathrm{QED}_{3}$. While the IR fates of these theories are unknown, they share the same anomaly with the deconfined critical point. So there is the possibility (among others) that either of them may flow to the deconfined critical point.

\section{A. Parton construction of $N_{f}=2 \mathbf{Q C D}_{3}$}

To see the connection between deconfined criticality and these theories, we now review the construction of the $\pi$-flux state on the square lattice [94] and demonstrate that its lowenergy theory, $\mathrm{QCD}_{3}$, has an emergent $\mathrm{SO}(5)$ symmetry. The Néel and VBS order parameters transform, as expected, as the five components of an $\mathrm{SO}(5)$ vector.

Consider the standard fermionic parton decomposition:

$$
\mathbf{S}_{i}=\frac{1}{2} f_{i \alpha}^{\dagger} \boldsymbol{\sigma}_{\alpha \beta} f_{i \beta}, \quad f_{\alpha i}^{\dagger} f_{\alpha i}=1,
$$

where $i$ labels a lattice site and the spin indices $\alpha, \beta$ are summed over. Let us form a matrix:

$$
X_{i}=\left(\begin{array}{cc}
f_{i \uparrow} & -f_{i \downarrow}^{\dagger} \\
f_{i \downarrow} & f_{i \uparrow}^{\dagger}
\end{array}\right) .
$$

The decomposition Eq. (84) is invariant under local $\mathrm{SU}(2)$ gauge rotations:

$$
\mathrm{SU}(2)_{g}: X_{i} \rightarrow X_{i}\left(U_{i}^{g}\right)^{\dagger} .
$$

The physical $\mathrm{SU}(2)$ spin rotations act as

$$
\mathrm{SU}(2)_{s}: X_{i} \rightarrow U^{s} X_{i} \text {, }
$$

and we can rewrite Eq. (84) as $\mathbf{S}_{i}=\frac{1}{4} \operatorname{tr}\left(X_{i}^{\dagger} \boldsymbol{\sigma} X_{i}\right)$. It will occasionally be convenient to write $f_{\uparrow}=(1 / \sqrt{2})\left(\chi_{0}+i \chi_{3}\right)$, $f_{\downarrow}=(1 / \sqrt{2})\left(-\chi_{2}+i \chi_{1}\right)$, i.e., $X=(1 / \sqrt{2})\left(\chi_{0}+i \chi_{a} \sigma^{a}\right)$, where $\chi_{m}, m=0,1,2,3$ are Majorana fermions. $\chi_{m}$ transforms as an $\mathrm{SO}(4)$ vector under the combined action of $\mathrm{SU}(2)_{s}$ and $\mathrm{SU}(2)_{g}$.

We consider a mean-field state on the square lattice,

$$
H_{\mathrm{MF}}=-\frac{i}{2} \sum_{i j} t_{i j} \chi_{i m} \chi_{j m}
$$

where $t_{i j}=-t_{j i}$, and $t_{i+\hat{x}, i}=1, t_{i+\hat{y}, i}=(-1)^{i_{x}}$, so that there is $\pi$ flux through each plaquette. This mean-field explicitly preserves the $\mathrm{SU}(2)_{s}$ and $\mathrm{SU}(2)_{g}$ symmetries, while lattice symmetries now act in a projective manner (see below). Each flavor $m$ of Majorana fermions produces two gapless Majorana cones, so the low-energy theory becomes

$$
H_{\mathrm{MF}}=i \sum_{v=1,2} \chi_{m, v}\left(\tau^{x} \partial_{x}-\tau^{z} \partial_{y}\right) \chi_{m, v},
$$

with $\tau$ acting on suppressed sublattice indices $\sigma \in A, B$ (see below). The index $v$ runs over two valleys, and lattice fields are related to continuum ones in the following way. The unit cell is doubled by $t_{i j}$. We label sites with even $i_{x}$ by $A$ and odd $i_{x}$ by $B$, and label unit cells by $\bar{i} \in(2 \mathbb{Z}+1 / 2, \mathbb{Z})$. Letting $\chi_{\bar{i}}=\left(\chi_{\bar{i}-\hat{x} / 2, A}, \chi_{\bar{i}+\hat{x} / 2, B}\right)$,

$$
\chi_{m, \bar{i}} \sim \tau^{x} \chi_{m, v=1}(x)+(-1)^{\bar{i}_{y}} \chi_{m, v=2}(x) .
$$

We can rewrite the mean-field Lagrangian as

$$
\mathcal{L}_{\mathrm{MF}}=i \bar{\chi}_{v, m} \gamma^{\mu} \partial_{\mu} \chi_{v, m},
$$

where $\bar{\chi}=\chi^{T} \gamma^{0}, \gamma^{0}=\tau^{y}, \gamma^{x}=i \tau^{z}, \gamma^{y}=i \tau^{x}$. The action of lattice symmetries is

$$
\begin{aligned}
T_{x} & : \chi \rightarrow \mu^{x} \chi, \\
T_{y} & : \chi \rightarrow \mu^{z} \chi, \\
R_{\pi / 2, A}: \chi & \rightarrow e^{\pi i \tau^{y} / 4} e^{-\pi i \mu^{y} / 4} \chi(-y, x), \\
P_{x, A}: \chi & \rightarrow \tau^{z} \mu^{z} \chi(-x, y), \\
T: \chi & \rightarrow \tau^{y} \mu^{y} \chi, \quad i \rightarrow-i,
\end{aligned}
$$

where $\mu$ acts on the valley index $v$ and spin or color indices $m$ have been suppressed. The subscript $A$ on $\pi / 2$ rotation $R$ and reflection $P$ indicates that these are around an $A$ site. These symmetries prohibit any quadratic term in $\chi$ with no derivatives in $\mathcal{L}_{\mathrm{MF}}$.

The mean-field theory has an $\mathrm{O}(8)$ symmetry acting on $m, v$. However, this is broken by fluctuations of $\mathrm{SU}(2)_{g}$ gauge field and four-fermi interactions. Let us first focus on the gauge field fluctuations. For this purpose it is convenient to introduce a $4 \times 2$ matrix, $X_{\alpha, v ; \beta}$, via

$$
X_{\alpha, v ; \beta}=\frac{1}{\sqrt{2}}\left(\chi_{0, v} \delta_{\alpha \beta}+i \chi_{a, v} \sigma_{\alpha \beta}^{a}\right) .
$$

The sublattice index is suppressed above. The Hermiticity of $\chi$ imposes an important relation:

$$
X^{*}=\sigma^{y} X \sigma^{y} .
$$

$\mathrm{SU}(2)$ spin and $\mathrm{SU}(2)$ gauge transformations act on $X$ from the left and right. The covariant derivative with respect to 
the dynamical $\mathrm{SU}(2)_{g}$ gauge field $a$ acts on $X$ as $D_{\mu}^{a} X=\partial_{\mu} X+i X a_{\mu}$, and $a$ transforms as

$$
\mathrm{SU}(2)_{g}: X \rightarrow X U_{g}^{\dagger}, \quad a_{\mu} \rightarrow U_{g} a_{\mu} U_{g}^{\dagger}-i \partial_{\mu} U_{g} U_{g}^{\dagger} .
$$

The Lagrangian including the dynamical gauge field then is

$$
\mathcal{L}_{\mathrm{QCD}_{3}}=i \operatorname{tr}\left(\bar{X} \gamma^{\mu} D_{\mu}^{a} X\right),
$$

with $\bar{X}=X^{\dagger} \gamma^{0}$. We see that Eq. (100) is invariant under a global symmetry,

$$
\operatorname{Sp}(4): X \rightarrow L X
$$

with $L \in \mathrm{Sp}(4)$-a unitary matrix acting on spin or valley indices $\alpha, v$ of $X_{\alpha, v ; \beta}$. The fact that $L$ lies in $\operatorname{Sp}(4)$, i.e., $L^{T} \sigma^{y} L=\sigma^{y}$, instead of in the larger group U(4) comes from the reality condition Eq. (98). The lattice symmetries in Eq. (96) are elements of this $\mathrm{Sp}(4)$ global symmetry combined with spatial symmetries of the Dirac theory. We note that the global symmetry $\mathrm{Sp}(4)$ and the gauge group $\mathrm{SU}(2)_{g}$ share a common nontrivial element: the center -1 . Thus, the physical global symmetry after modding out by $\mathrm{SU}(2)_{g}$ is actually $\mathrm{Sp}(4) / Z_{2}=\mathrm{SO}(5)$ [it is useful to recall that $\mathrm{Sp}(4)=\operatorname{Spin}(5)]$. An order parameter for this $\mathrm{SO}(5)$ symmetry is given by a five-component vector:

$$
n^{a}=\operatorname{tr}\left(\bar{X} \Gamma^{a} X\right),
$$

with $\boldsymbol{\Gamma}=\left\{\mu^{z},-\mu^{x}, \sigma^{x} \mu^{y}, \sigma^{y} \mu^{y}, \sigma^{z} \mu^{y}\right\}$. The first two components, $n^{1}, n^{2}$, have precisely the transformation properties of the $x$ and $y$ components of the VBS order parameter, while the last three components $\operatorname{tr}\left(\bar{X} \sigma^{a} \mu^{y} X\right)$ correspond to the Néel order parameter.

We note in passing that if we want to be less explicit about the full emergent symmetry of Eq. (100), we can express the Lagrangian in terms of two flavors of $\mathrm{SU}(2)_{g}$ charged complex Dirac fermions, $\psi_{\alpha, v}=i \sigma_{\alpha, \beta}^{y} X_{1, v ; \beta}$, with $\alpha$ being the color index, and

$$
\mathcal{L}=i \bar{\psi}_{v} \gamma^{\mu}\left(\partial_{\mu}-i a_{\mu}\right) \psi_{v},
$$

with $\bar{\psi}_{v}=\psi_{v}^{\dagger} \gamma^{0}$. In other words, this theory is $N_{f}=2 \mathrm{QCD}_{3}$.

There are (at least) three possible scenarios for this theory. First, $N_{f}=2 \mathrm{QCD}_{3}$ could confine, and in the process spontaneously break $\mathrm{SO}(5)$ symmetry by generating a condensate $\left\langle n^{a}\right\rangle \neq 0$. In the setting of the spin system, quartic terms in the Lagrangian will then select either the VBS state or the Néel state. This is the boring scenario.

Second, $N_{f}=2 \mathrm{QCD}_{3}$ could, in principle, flow to a stable gapless fixed point at which all perturbations (e.g., four-fermi couplings and velocity anisotropies) that preserve lattice and $\mathrm{SO}(3)_{s}$ symmetries are irrelevant. We would then have a completely stable gapless spin-liquid phase with emergent $\mathrm{SO}(5)$ symmetry. [In principle, QCD could also flow to a gapped $\mathrm{SO}(5)$-invariant spin liquid; as shown in Sec. VIE, this is possible only if time-reversal symmetry is broken.]

Third, $N_{f}=2 \mathrm{QCD}_{3}$ could flow to a gapless fixed point which is stable in the presence of $\mathrm{SO}(5)$, but which allows a single relevant perturbation when $\mathrm{SO}(5)$ is broken to the physical symmetry: the operator $\mathcal{O}_{1}$ in Sec. IV E [breaking $\mathrm{SO}(5)$ to $\left.\mathrm{SO}(3)_{s} \times \mathrm{SO}(2)_{\mathrm{VBS}}\right]$. Then $N_{f}=2 \mathrm{QCD}_{3}$ tuned to an $\mathrm{SO}(5)$-symmetric point describes the deconfined critical point, and perturbing it by $\mathcal{O}_{1}$ drives it into either the VBS phase or the Néel phase. This is the scenario relevant for this paper.

\section{B. Higgs descendent: $N_{f}=4$ compact $Q E D_{3}$}

Starting from $N_{f}=2 \mathrm{QCD}_{3}$, we now Higgs the gauge group from $\mathrm{SU}(2)$ down to $\mathrm{U}(1)$. We introduce and condense a scalar field $\phi$ that transforms as a spin-1 vector under $\mathrm{SU}(2)_{g}$ and as a scalar under $\mathrm{SO}(5)$ (such a field is allowed in the theory). After a charge-conjugation redefinition of half of the Dirac fermions, the resulting theory is

$$
\mathcal{L}=\sum_{i=1}^{4} i \bar{\psi}_{i} \gamma^{\mu}\left(\partial_{\mu}-i a_{\mu}\right) \psi_{i}+\left(\lambda \mathcal{M}_{a}+\text { H.c. }\right),
$$

where $a_{\mu}$ is now a U(1) gauge field, and the monopole operator $\mathcal{M}_{a}$ represents instanton events that change the flux of $a_{\mu}$ by $2 \pi$. In general, such a term should be expected when the U(1) gauge field comes from Higgsing of a higher gauge symmetry. In condensed-matter language [95] such theories are called compact $\mathrm{QED}_{3}$.

The fermion fields $\psi_{i}$ transform as a spinor representation under the global $\mathrm{SO}(5)$ - this follows simply from the symmetry properties of $\mathrm{QCD}_{3}$. Naively one might expect the Lagrangian Eq. (104) to have a larger flavor symmetry, say, SU(4), respected by the Dirac term. However, it turns out that the monopole term breaks the symmetry down to $\mathrm{SO}(5)$. This can be seen by analyzing the fermion zero modes [65] associated with the monopole operator $\mathcal{M}_{0 a}$ : each Dirac fermion $\psi_{i}$ contributes a complex fermion zero mode $f_{i}$ in the monopole background, and gauge invariance requires two of the four zero modes to be filled in the ground state, so a gauge-invariant operator should be represented as $f_{i}^{\dagger} f_{j}^{\dagger} \mathcal{M}_{0 a}$. There are in total six of them, and it is straightforward to check that they split into $6=$ $1 \oplus 5$ with respect to the $\mathrm{SO}(5)$ symmetry. The monopole operator that appears in the Lagrangian in Eq. (104) is precisely the $\mathrm{SO}(5)$ scalar monopole. It transforms nontrivially under higher flavor symmetries, and $\mathrm{SO}(5)$ is the maximal flavor group that is compatible with it.

Since this $N_{f}=4$ compact $\mathrm{QED}_{3}$ is just a Higgs descendent of $N_{f}=2 \mathrm{QCD}_{3}$, they must have the same anomaly structure. Therefore, they share the same set of possible IR behaviors, including those discussed at the end 
of Sec. VIA. Of course, the two theories could pick different choices.

\section{INTERPRETATION AS SURFACE THEORY OF $(3+1)$ D BOSON SPT}

Here, we show that $\mathrm{SO}(5)$-symmetric $N_{f}=2 \mathrm{QCD}_{3}$ (and hence $N_{f}=4$ compact $\mathrm{QED}_{3}$ ) can be interpreted as a surface theory of a bosonic SO(5)-protected $(3+1) \mathrm{D} \mathrm{SPT}$ phase. This statement is independent of the dynamics of $\mathrm{QCD}_{3}$ : it remains true even if the theory spontaneously breaks $\mathrm{SO}(5)$. To make this statement, we have to understand how an $\mathrm{SO}(5)$ background gauge field $\mathcal{A}^{5}$ enters in $\mathrm{QCD}_{3}$ and show that this theory has an anomaly, which is precisely compensated by the $(3+1)$ D SPT bulk. Here, we establish this using a physical argument. In Sec. VII, we provide a precise formal proof.

Let us first determine the anomaly of this theory by thinking about a background gauge field that couples to the $\mathrm{SO}(5)$ currents. An $\mathrm{SO}(5)$ gauge field $\mathcal{A}^{5}$ admits $Z_{2^{-}}$ indexed monopole configurations, since $\pi_{1}(\mathrm{SO}(5))=$ $Z_{2}$. In $(2+1) \mathrm{D}$ these correspond to instanton events, and we can ask whether there is anything nontrivial about them.

We examine a monopole background of the following form. Consider an $\mathrm{SO}(2) \times \mathrm{SO}(3)$ subgroup of $\mathrm{SO}(5)$, with $\mathrm{SO}(2)$ acting on the first two components of vector $n^{a}$ in Eq. (102) and $\mathrm{SO}(3)$ on the last three. Place a unit magnetic monopole in the $\mathrm{SO}(2)$ subgroup, i.e.,

$$
\mathcal{A}_{\mu}^{5}=\mathcal{A}_{\mu}^{\operatorname{mon}}(x) T^{1},
$$

where $T^{1}$ is the generator of $\mathrm{SO}(2)$ and $\mathcal{A}^{\text {mon }}$ is the standard potential associated with a unit magnetic monopole. In the presence of such a background $\mathcal{A}^{5}$ field, only a subgroup of the global $\mathrm{SO}(5)$ symmetry survives: these are rotations in an $\mathrm{SO}(3)$ subgroup (whose generators commute with $T^{1}$ ) and the $\mathrm{SO}(2)$ rotations generated by $T^{1}$ itself.

The following argument provides a hint of the properties of the instanton. Rather than considering the $\mathrm{QCD}_{3}$ theory directly, suppose we add in an extra field $\hat{n}$, transforming in the vector representation of $\mathrm{SO}(5)$, that couples to fermion bilinears through a Yukawa coupling. In the limit that this coupling is strong, we can integrate out the fermions, and standard methods $[28,29,96]$ produce an $\mathrm{SO}(5)$ nonlinear sigma model in the $\hat{n}$ field with a level-1 WZW term (Sec. II A). Now the SU(2) gauge field does not couple directly to any matter field, and is expected to confine at low energy, leaving behind the SO(5) WZW model as the remaining nontrivial theory. Indeed, this supports the idea that $\mathrm{QCD}_{3}$ correctly describes the Néel-VBS intertwinement in square lattice quantum magnets. Physically the $\mathrm{SO}(5)$ instanton in Eq. (105) has the effect of creating a vortex in two components of the $\hat{n}$ field. We now ask how this vortex transforms under the unbroken symmetry $\mathrm{SO}(2) \times \mathrm{SO}(3)$. The vortex carries no charge under
$\mathrm{SO}(2)$, but we know that the vortex transforms as a spinor under $\mathrm{SO}(3)$ due to the WZW term. We conclude that the instanton configuration described above transforms as an $\mathrm{SO}(3)$ spinor with zero $\mathrm{SO}(2)$ charge also.

For a conventional $\mathrm{SO}(5)$ sigma model (i.e., without the WZW term) the $\mathrm{SO}(5)$ instanton will transform trivially under $\mathrm{SO}(2) \times \mathrm{SO}(3)$. The projective transformation of the instanton under the $\mathrm{SO}(3)$ subgroup tells us that in the presence of the level-1 WZW term the SO(5) symmetry is realized anomalously. It cannot be realized as the on-site symmetry of any strictly $(2+1) \mathrm{D}$ model. Clearly, the same instanton structure also characterizes the $\mathrm{QCD}_{3}$ theory.

This is the physics of the desired anomaly. Note also that this instanton operator is bosonic (i.e., in relativistic parlance it has spin 0 under spatial rotations).

It is instructive to rederive the instanton structure of $\mathrm{QCD}_{3}$ directly from the UV Majorana fermion theory. We now briefly indicate how this works out. It is important to recognize that the fermions that enter $\mathrm{QCD}_{3}$ transform as a fundamental of $\mathrm{Sp}(4)$, although the physical global symmetry is $\mathrm{SO}(5)=\mathrm{Sp}(4) / Z_{2}$. We therefore need to lift the $\mathrm{SO}(5)$ gauge field $\mathcal{A}^{5}$ to an $\operatorname{Sp}(4) A^{5}$, which enters the theory Eq. (100) via $D_{\mu} X \rightarrow\left(\partial_{\mu}-i A_{\mu}^{5}\right) X+i X a_{\mu}$. For instance, consider

$$
A^{5}=\frac{\mathcal{A}^{\mathrm{mon}}}{2} \mu^{y},
$$

where $\mu$ are Pauli matrices that act on the valley index. Naively this may seem to require creating a $\pi$ flux through the nonzero component of $A^{5}$ which apparently violates Dirac quantization for the monopole. However, we should remember that we also have a dynamical SU(2) gauge field $a$ that the fermions are coupled to: if the lift to $\operatorname{Sp}(4)$ is accompanied by a $\pi$-flux instanton in one of the three components of the SU(2) gauge field, then we have a sensible configuration that satisfies Dirac quantization [97]. For instance, we may give the dynamical gauge field a background value $a=a^{3} \sigma^{3}$, with

$$
a^{3}=\frac{\mathcal{A}^{\text {mon }}}{2} .
$$

It is then convenient to rewrite Eq. (100) in terms of a single color component of $X$, e.g., $X_{\alpha v, \uparrow}$ :

$\mathcal{L}=i \bar{X}_{\alpha v, \uparrow} \gamma^{\mu}\left[\left(\partial_{\mu}+i a_{\mu}^{3}\right) \delta_{\alpha \alpha^{\prime}} \delta_{v v^{\prime}}-i A_{\alpha, v ; \alpha^{\prime}, v^{\prime}}^{5}\right] X_{\alpha^{\prime} v^{\prime}, \uparrow}$.

Observe that two Dirac fermions $X_{\alpha-}$ with $\mu^{y}=-1$ see a $2 \pi$-flux instanton, and another two Dirac fermions $X_{\alpha+}$ with $\mu^{y}=+1$ see no background flux (we drop the color index $\uparrow$ here). Further, each pair of Dirac fermions transforms as a spin $1 / 2$ under the global $\mathrm{SO}(3)_{s}$ subgroup left unbroken by the $\mathrm{SO}(5)$ monopole. In the language of the state-operator correspondence, in the presence of the 
instanton background the $X_{\alpha-}$ fermions will form two zero modes. Charge neutrality with respect to the color gauge field $a^{3}$ then implies that we occupy one of these zero modes. Thus, the instanton will transform as an $\mathrm{SO}(3)$ spinor in agreement with the arguments above. It is also easy to see that it has zero charge under the unbroken $\mathrm{SO}(2)$ subgroup of the global $\mathrm{SO}(5)$ symmetry.

Next, we want to show that this nontrivial instanton structure is consistent at the surface of a $(3+1) \mathrm{D}$ boson SPT with $\mathrm{SO}(5)$ symmetry. In other words, we can regularize $\mathrm{QCD}_{3}$ with its full $\mathrm{SO}(5)$ symmetry as an onsite symmetry at the boundary of a $(3+1) \mathrm{D}$ bosonic SPT phase.

First, let us discuss possible $\mathrm{SO}(5)$-symmetric boson SPTs in $(3+1)$ D. Consider any short-range entangled gapped phase of an $\mathrm{SO}(5)$-symmetric boson theory, and again couple in background $\mathrm{SO}(5)$ gauge fields. The bulk again admits $Z_{2}$-indexed monopoles in this gauge field, which can be chosen to break $\mathrm{SO}(5)$ to $\mathrm{SO}(2) \times \mathrm{SO}(3)$.

Now there are logically two sharply distinct possibilities: does the monopole transform as a spinor under the unbroken $\mathrm{SO}(3)$ symmetry or not? If it is a spinor, then the original gapped state is a SPT state. The other question we may ask is what the charge is under the unbroken $\mathrm{SO}(2)$ symmetry. This charge can be continuously tuned by changing the $\operatorname{SO}(5) \theta$ angle:

$$
\mathcal{L}=\frac{\theta}{4(2 \pi)^{2}} \operatorname{Tr}_{\mathrm{SO}(5)}\left(\mathcal{F}^{5} \wedge \mathcal{F}^{5}\right),
$$

where $\mathcal{F}_{\mu \nu}^{5}=\partial_{\mu} \mathcal{A}_{\nu}^{5}-\partial_{\nu} \mathcal{A}_{\mu}^{5}-i\left[\mathcal{A}_{\mu}^{5}, \mathcal{A}_{\nu}^{5}\right]$ is the $\mathrm{SO}(5)$ field strength. Since $\theta$ is a continuous parameter, in the absence of additional symmetries (e.g., time reversal) it does not label a distinct SPT phase. It is crucial to note that changing $\theta$ does not affect the $\mathrm{SO}(3)$ transformation properties of the monopole. Thus, the SO(5) SPT where the SO(2) monopole is an $\mathrm{SO}(3)$ spinor is an SPT rather distinct from the more familiar boson and fermion topological insulators protected by $\mathrm{U}(1)$ and time-reversal symmetries. Below, we discuss the topological action for this SPT. Finally, we may ask whether the monopole is a boson or fermion [98]. This property may be altered by shifting $\theta \rightarrow \theta+2 \pi[99,100]$.

Now, let us assume the monopole is a boson that carries no $\mathrm{SO}(2)$ charge. Then if it does not transform as a spinor under $\mathrm{SO}(3)$, the original gapped state is totally trivial. If it is a spinor under $\mathrm{SO}(3)$, then the original gapped state is a SPT state which has the exact same monopole structure to compensate for the instanton structure of $\mathrm{QCD}_{3}$ with $N_{f}=2$ as a potential boundary state.

\section{Explicit constructions for the SO(5) SPT}

First, let us argue that such a $(3+1)$ D SPT state indeed exists in a system of $\mathrm{SO}(5)$-symmetric bosons by a coupled layer construction. Notice that though $N_{f}=2 \mathrm{QCD}_{3}$ has an anomalous $\mathrm{SO}(5)$ symmetry, the anomaly disappears if we take two copies of it [101]. This is because the $\mathrm{SO}(5)$ monopole (instanton) then gets a spin $1 / 2$ [under $S O(3)$ ] from each copy and hence can always be made trivial.

We can now construct the required $(3+1) \mathrm{D}$ bosonic SPT state by starting with a stack of $2 \mathrm{D}$ layers, each containing two copies of $\mathrm{QCD}_{3}$. We take one copy from one layer and trivialize it by pairing with another copy from the next layer. This will give a trivial gapped bulk, but at the boundary we are left with a single copy of $\mathrm{QCD}_{3}$.

We can also construct the bulk boson SPT more explicitly using fermionic partons, following a similar approach to Refs. [102,103]. Consider first a $(3+1) \mathrm{D}$ fermionic toplogical superconductor with $\mathrm{SO}(8) \times Z_{2}^{T}$ symmetry. A continuum model for this state consists simply of 8 relativistic, free, massive Majorana fermions,

$$
\mathcal{L}=\sum_{i=1}^{8} \bar{\chi}_{i}\left(i \gamma^{\mu} \partial_{\mu}+m\right) \chi_{i},
$$

with $\bar{\chi}_{i}=\chi_{i}^{T} \gamma^{0}$. For one sign of the Majorana mass $m$ we will have a trivial gapped state, while for the other sign we will have a topological superconductor. The $(2+1) \mathrm{D}$ surface of this free-fermion state correspondingly has 8 massless Majorana cones. Now let us couple this system to a dynamical $\mathrm{SU}(2)$ gauge field $a$. As in our $(2+1) \mathrm{D}$ discussion, we label the 8 Majoranas $\chi$ by indices $m=0,1,2,3, v=1,2$, and form the field $X_{\alpha, v ; \beta}$ in Eq. (97). The SU(2) gauge symmetry acts on $X$ from the right, as in Eq. (86), and the $(3+1) \mathrm{D}$ gauge action has the form

$$
\mathcal{L}_{\mathrm{QCD}_{4}}=\operatorname{tr}\left[\bar{X}\left(i \gamma^{\mu} D_{\mu}^{a}+m\right) X\right] .
$$

In the bulk this gauge theory describes a bosonic system with $\mathrm{SO}(5) \times Z_{2}^{T}$ symmetry. $\mathrm{SO}(5)$ is realized projectively on the Majorana fermions, which form an $\mathrm{Sp}(4)$ fundamental Eq. (101). As in $(2+1) \mathrm{D}$ [Eq. (103)], we can rewrite Eq. (111) as two flavors of Dirac fermions with the same mass $m$ coupled to an $\mathrm{SU}(2)$ gauge field.

What state does the theory Eq. (111) realize? First, consider this theory on a closed manifold. Then integrating out the massive fermions produces, at long wavelengths, the standard Yang-Mills action for the dynamical SU(2) gauge field with no topological term. Indeed, each flavor of Dirac fermions with inverted mass would give rise to an $\mathrm{SU}(2) \theta$ term in the action with $\theta=\pi$ :

$$
\mathcal{L}_{\theta}=\frac{\theta}{2(2 \pi)^{2}} \operatorname{tr}_{\mathrm{SU}(2)} f \wedge f .
$$

Thus, two flavors of Dirac fermions with the same mass give $\theta=2 \pi$, which is equivalent to $\theta=0$ (see Sec. VII for a more careful discussion). It is expected that the pure Yang-Mills theory will confine, and so the ground state is seemingly trivial. Now consider the theory in the presence 
of a boundary. Though the bulk is confined, the boundary is precisely the $\mathrm{QCD}_{3}$ theory of interest to us with global $\mathrm{SO}(5) \times Z_{2}^{T}$ symmetry. As promised, the bulk theory therefore describes the SPT phase of bosons with $\mathrm{SO}(5) \times Z_{2}^{T}$ symmetry. In principle, this construction could also be used to write a variational (Gutzwiller-projected) wave function for a lattice $\mathrm{SO}(5)$ topological paramagnet [104].

How do we formally describe the bulk response to a background $\mathrm{SO}(5)$ gauge field that captures the structure of the $Z_{2}$-indexed monopole in these systems? In Sec. VII we show that the partition function of the bulk SPT phase takes the form

$$
Z\left[\mathcal{A}^{5}\right]=\left|Z\left[\mathcal{A}^{5}\right]\right| e^{i \pi \int w_{4}\left[\mathcal{A}^{5}\right]} .
$$

Here, $w_{4}\left[\mathcal{A}^{5}\right]=0,1$ is a quantity known as the fourth Steifel-Whitney class of the $\mathrm{SO}(5)$ gauge bundle $\mathcal{A}^{5}$ [105]. The phase $e^{i \pi \int w_{4}\left[\mathcal{A}^{5}\right]}$ is the analog of the familiar $\theta$-term response of the standard topological insulator to background $\mathrm{U}(1)$ gauge fields. In contrast to the usual case, here the $\theta$ angle is restricted to two discrete values: 0 (corresponding to a totally trivial state) or $\pi$ (corresponding to the SPT phase of interest to us here). Precisely such a discrete $\theta$ term was introduced a few years ago in Ref. [44] for nonAbelian gauge theories. In that work the possibility of such $\theta$ terms was pointed out and some of their physical consequences were discussed. We see that such $\theta$ terms emerge naturally in the response of bosonic SPT phases. Our discussion above can be viewed as a construction of an $\mathrm{SO}(5)$-symmetric bosonic $(3+1) \mathrm{D}$ model whose response includes these discrete $\theta$ terms. Indeed, in Sec. VII, we show explicitly that the theory Eq. (111) has this discrete $\theta$ term in its response to a background $\mathrm{SO}(5)$ gauge field.

\section{E. Symmetry-enforced gaplessness}

As mentioned above, the IR fate of $N_{f}=2 \mathrm{QCD}_{3}$ is at present unclear. However, our understanding of the anomalous symmetry realization in this theory enables us to derive some general restrictions. We show that either the $\mathrm{SO}(5) \times Z_{2}^{T}$ symmetry is spontaneously broken or the theory is gapless in the IR. This result follows purely from the anomalous symmetry realization. Indeed, it is a general feature of the surface of the bulk $(3+1) \mathrm{D}$ boson SPT with $\mathrm{SO}(5) \times Z_{2}^{T}$ symmetry discussed in the previous section. Such a phenomenon was first described for some fermion SPTs in Ref. [106] and dubbed "symmetry-enforced gaplessness." Other examples, including some boson SPTs, are described in Refs. [107,108].

Consider a putative gapped state of the $(2+1) \mathrm{D}$ theory that preserves the $\mathrm{SO}(5) \times Z_{2}^{T}$ symmetry. All the quasiparticles of this state must transform under some representation, possibly projective. As usual, if a quasiparticle transforms nonprojectively under $\mathrm{SO}(5)$, we can "screen" it using composites of the $\hat{n}$ vector to make it a singlet under
$\mathrm{SO}(5)$. Therefore, the only nontrivial symmetry possibility is a quasiparticle transforming under the four-dimensional spinor representation [fundamental of $\mathrm{Sp}(4)$ ]. Let us call such quasiparticles $X_{I}$.

We think of $\mathrm{QCD}_{3}$ as living on the surface of the $(3+1) \mathrm{D}$ SPT described above. Now let us tunnel in a $Z_{2}$-valued $\mathrm{SO}(5)$ monopole through the surface. We know that the monopole breaks $\mathrm{SO}(5)$ to $\mathrm{SO}(2) \times \mathrm{SO}(3)$, and that it transforms in the $(0,1 / 2)$ representation of $\mathrm{SO}(2) \times \mathrm{SO}(3)$. Therefore, in order for $\mathrm{SO}(2) \times \mathrm{SO}(3)$ charge to be conserved, there must be a quasiparticle in this putative gapped surface state with these properties. However, the only quasiparticles that transform nontrivially under $\mathrm{SO}(5)$ are the "spinors" $X_{I}$. They transform with $\mathrm{SO}(2)$ charge of $1 / 2$ and as a spinor under the $\mathrm{SO}(3)$, and not under the $(0,1 / 2)$ representation. It follows that the gapped state we imagined cannot have the right anomaly, and hence is not a possible surface state.

Note, however, that if time reversal is broken, then there can be a Hall conductivity for the $\mathrm{SO}(5)$ currents. Then the $\mathrm{SO}(5)$ monopole threading will nucleate an $\mathrm{SO}(2)$ charge determined by the Hall conductivity. This can then combine with the $\mathrm{SO}(5)$ spinor $X_{I}$ to produce an object with $(0,1 / 2)$ quantum numbers under the $\mathrm{SO}(2) \times \mathrm{SO}(3)$ symmetry, as required. Thus, if time reversal is broken, a gapped $\mathrm{SO}(5)-$ symmetry-preserving state is no longer prohibited. Indeed, it is easy to construct a "chiral spin-liquid" state explicitly; see Sec. VII D.

The conclusion therefore is that, in the IR, $N_{f}=2 \mathrm{QCD}_{3}$ with $\mathrm{SO}(5) \times Z_{2}^{T}$ symmetry must either spontaneously break the symmetry or be gapless, i.e., flow to a CFT. It cannot be fully gapped while preserving symmetries even if we allow for nontrivial topological order.

As another application of this result, consider the fate of the Néel-VBS transition at the longest distances. One interpretation of the existing numerics is to say that the renormalization group (RG) flows are attracted to a ray with $\mathrm{SO}(5) \times Z_{2}^{T}$ symmetry. If so, then the eventual destination of this ray is either a weak first-order transition, a $Z_{2}^{T}$ broken spin liquid, or a gapless CFT - a gapped, symmetrypreserving, topologically ordered state is ruled out [109].

\section{QCD 3 AS THE SURFACE OF AN SO(5)-INVARIANT $(3+1)$ D SPT: FORMAL DESCRIPTION}

Here, we expand on the discussion in Sec. VIC and demonstrate more formally that $\mathrm{SO}(5)$-symmetric $N_{f}=2$ $\mathrm{QCD}_{3}$, Eq. (100), can be interpreted as a surface theory of a bosonic $\mathrm{SO}(5)$-protected $(3+1) \mathrm{D}$ SPT phase. We first develop a precise formal description of the anomaly of $\mathrm{QCD}_{3}$ and show how it is compensated by the $(3+1) \mathrm{D}$ SPT bulk. We also sharpen the parton construction of this SPT outlined in Sec. VIC to derive the bulk partition function in the presence of a background $\mathrm{SO}(5)$ gauge field. We explicitly derive the advertised discrete theta term. See 
Appendix D for a brief summary of math concepts that will be useful in this section and Appendix $\mathrm{E}$ for a review of the field-theoretic description of topological superconductors.

\section{A. Gauging $\operatorname{SO}(3)_{s}$}

First, it is useful to recall how the gauge field associated with the $\mathrm{SO}(3)_{s}$ spin-rotation symmetry enters in $\mathrm{QCD}_{3}$. Since $\mathrm{SO}(3)_{s}$ symmetry is a true on-site symmetry of the lattice spin system, its implementation in $\mathrm{QCD}_{3}$ must be nonanomalous. Given an $\mathrm{SO}(3)_{s}$ gauge bundle $E_{s}$ over our spacetime 3 manifold $M$, [110] we attempt to lift it to an $\mathrm{SU}(2)_{s}$ gauge bundle $\hat{E}_{s}$. The resulting $\mathrm{SU}(2)_{s}$ transition functions $\hat{U}_{i j}^{s}$ might fail to satisfy the cocycle condition: $\hat{U}_{i k}^{s} \hat{U}_{k j}^{s} \hat{U}_{j i}^{s}=(-1)^{w_{2}\left(E_{s}\right)_{k j i}}$, where $w_{2}\left(E_{s}\right) \in H^{2}\left(M, Z_{2}\right)$ is a representative of the second Stiefel-Whitney class of $E_{s}$ [111]. In this case, we take the transition functions of our dynamical gauge field $\mathrm{SU}(2)_{g}$ to violate the cocyle condition by precisely the same factor in the center of $\mathrm{SU}(2)_{g}$. That is, if we project the $\mathrm{SU}(2)_{g}$ transition functions $\hat{U}^{g}$ to $\mathrm{SO}(3)_{g}$, we get an $\mathrm{SO}(3)$ bundle $E_{g}$ with $w_{2}\left(E_{g}\right)=w_{2}\left(E_{s}\right)$. The fermions $\chi_{m, v}$ then see an $\mathrm{SO}(4)=$ $\left[\mathrm{SU}(2)_{s} \times \mathrm{SU}(2)_{g}\right] / Z_{2}$ gauge field which satisfies the cocycle condition. In the background of this $\mathrm{SO}(4)$ gauge field $A^{s, g}$, the action Eq. (100) becomes

$$
L=i \bar{\chi}_{v} \gamma^{\mu}\left(\partial_{\mu}-i A^{s, g}\right) \chi_{v},
$$

where $A^{s, g}$ lives in so(4) Lie algebra and acts on the spincolor index $m$ of $\chi_{m, v}$ but not on the valley index $v$. We note that in addition to the so(4) gauge field, the fermions $\chi_{m, v}$ also see a spin structure (we suppress the spin connection above) with transition functions $h_{i j} \in \operatorname{Spin}(3)$. Every three-manifold is spin, so $h_{i j}$ can always be chosen to satisfy the cocycle condition (as we assume in the discussion above). However, it will be more useful to think of $h_{i j}$ as arbitrary lifts to $\mathrm{Spin}(3)$ of the $\mathrm{SO}(3)$ transition functions in the tangent bundle. The fermions see only a combination of $\mathrm{SU}(2)_{s}, \mathrm{SU}(2)_{g}$, and $\mathrm{Spin}(3)_{T M}$ transition functions, so we require only this combination to satisfy the cocycle condition [112]. A change $h_{i j} \rightarrow \zeta_{i j} h_{i j}, \zeta_{i j}= \pm 1$, can be compensated by modifying the $\mathrm{SU}(2)_{g}$ bundle, $\hat{U}_{i j}^{g} \rightarrow \zeta_{i j} \hat{U}_{i j}^{g}$, so the theory does not depend on the spin structure. Further, $w_{2}\left(E_{s}\right)+w_{2}\left(E_{g}\right)+w_{2}(T M)=0(\bmod 2)$, where we are now thinking of $w_{2}$ 's as concrete cochains representing $H^{2}\left(M, Z_{2}\right)$ [as already noted, as a cohomology class, $\left.w_{2}(T M)=0\right]$.

We now discuss the regularization of Eq. (114) - in principle, such regularization is provided by the lattice we started with. An equivalent continuum regularization is obtained by using Pauli-Villars (PV) regulators with opposite mass for the Majoranas in the two valleys $v=1,2$. Recall that for a single $\mathrm{SO}(n)$ vector Majorana fermion coupled to an $\mathrm{SO}(n)$ gauge field $A$, the $\mathrm{PV}$ regulated partition function is given by [60]

$$
Z_{\chi ; \mathrm{PV}, \pm}(A)=\left|Z_{\chi}(A)\right| \exp \left[\mp \pi i \eta\left(i D_{A}\right) / 4\right],
$$

where the sign in the exponent is determined by the sign of the Pauli-Villars mass. Here, $\eta\left(i D_{A}\right)$ is the $\eta$ invariant of the Dirac operator $i D_{A}=i \gamma^{\mu}\left(\partial_{\mu}+i \omega_{\mu}-i A_{\mu}\right)$, [113]

$$
\begin{aligned}
\eta & =\eta(0)+N_{0}, \\
\eta(s) & =\sum_{\lambda \neq 0} \operatorname{sgn}(\lambda)|\lambda|^{-s},
\end{aligned}
$$

where $\lambda$ in the above sum are eigenvalues of $i D_{A}$, and $N_{0}$ are the number of zero modes of $i D_{A}$. So, when we use PV regulators of opposite mass for the two valleys $v=1,2$, we obtain, after integrating the Majorana fermions out,

$$
\exp \left(-S_{\mathrm{QCD}_{3}}\left[A^{s, g}\right]\right)=\left|Z_{\chi_{v=1}}\left(A^{s, g}\right)\right|^{2},
$$

with $Z_{\chi_{v=1}}\left(A^{s, g}\right)$ the partition function of Majoranas in just a single valley $v=1$. The theory thus defined obviously preserves $\mathrm{SO}(3)_{s}$ as a nonanomalous symmetry. Likewise, time-reversal symmetry [last line of Eq. (96)] is preserved and nonanomalous - this must be the case, as it is an on-site symmetry of the initial lattice model [115]. The discrete lattice symmetries in Eq. (96) are global symmetries of Eq. (117); however, they are (in a certain sense) anomalous: there is no contradiction here, since they are not realized by the original lattice model in an on-site manner.

For future reference, we note that we can obtain an equivalent theory [Eq. (117)] by regulating both valleys in the same way (with the same sign of PV mass) and supplementing the action by a Chern-Simons term for $A^{s, g}$ :

$$
\begin{aligned}
S_{\mathrm{QCD}_{3}}= & \int_{M}\left[\bar{\chi}_{v} \gamma^{\mu}\left(\partial_{\mu}+i \omega_{\mu}-i A^{s, g}\right) \chi_{v}\right]_{\mathrm{PV},+} \\
& -i \mathrm{CS}_{\mathrm{SO}(4)}\left[A^{s, g}, Y_{4}\right]-4 i \mathrm{CS}_{g}\left[Y_{4}\right],
\end{aligned}
$$

where the subscript $\mathrm{PV}_{+}$indicates that the $\mathrm{PV}$ mass is the same for both valleys; see Eq. (115). We use the notation where $\mathrm{CS}_{\mathrm{SO}(n)}\left[A, Y_{4}\right]$ is the Chern-Simons action for $\mathrm{SO}(n)$ gauge field $A$ at level 1, [116] and $\operatorname{CS}_{g}\left[Y_{4}\right]$ is the gravitational Chern-Simons action (corresponding to the gravitational response of a $p_{x}+i p_{y}$ superconductor). The significance of the parameter $Y_{4}$ is as follows. We recall that a technical trick to define a Chern-Simons term is to extend the three-manifold $M$ to a four-manifold $Y_{4}$, and also extend the gauge field $A$ from $M$ to $Y_{4}$ :

$$
\begin{gathered}
\mathrm{CS}_{\mathrm{SO}(n)}\left[A, Y_{4}\right]=\frac{\pi}{2(2 \pi)^{2}} \int_{Y_{4}} \operatorname{tr}_{\mathrm{SO}(n)} F \wedge F, \\
\mathrm{CS}_{g}\left[Y_{4}\right]=\frac{\pi}{8\left(24 \pi^{2}\right)} \int_{Y_{4}} \operatorname{tr} R \wedge R,
\end{gathered}
$$


where $F=d A-i A \wedge A$ is the $\mathrm{SO}(n)$ field strength, $R$ is the Riemann curvature tensor, and the $\operatorname{trace} \operatorname{tr}_{\mathrm{SO}(n)}$ is in the $n$-dimensional vector representation. In order for a theory to be a well-defined strictly $(2+1) \mathrm{D}$ theory, it must be independent of the choice of the four-manifold $Y_{4}$ and the particular extension of the gauge field to $Y_{4}$. For our theory, Eq. (118), this is actually guaranteed by the Atiyah-PatodiSinger (APS) theorem. Indeed, recall that by the APS theorem, if our three-manifold $M$ is endowed with an $\left[\mathrm{SO}(n)_{A} \times \operatorname{Spin}(3)_{T M}\right] / Z_{2}$ bundle $E$ (where $n$ is even) and $M$ is the boundary of a four-manifold $Y_{4}$ such that $E$ extends to a $\left[\mathrm{SO}(n)_{A} \times \operatorname{Spin}(4)_{T Y_{4}}\right] / Z_{2}$ bundle over $Y_{4}$, then [114]

$\frac{\pi}{2} \eta\left(i D_{A, M}^{\mathrm{SO}(n)}\right)=\mathrm{CS}_{\mathrm{SO}(n)}\left[A, Y_{4}\right]+n \mathrm{CS}_{g}\left[Y_{4}\right]-2 \pi \mathcal{J}\left[A, Y_{4}\right]$,

where $2 \mathcal{J}\left[A, Y_{4}\right]$ is the index of the Dirac operator $i D_{A}$ on $Y_{4}$ with APS boundary conditions [117]. Since the left-hand side of Eq. (120) depends only on the boundary data, the sum $\mathrm{CS}_{\mathrm{SO}(n)}\left[A, Y_{4}\right]+n \mathrm{CS}_{g}\left[Y_{4}\right]$ is independent of the extension chosen modulo $2 \pi$ [118]. This means that Eq. (118) is a well-defined strictly $(2+1) \mathrm{D}$ theory. Furthermore, integrating the fermions in Eq. (118) out using Eq. (115) and applying Eq. (120), we recover the original regularization Eq. (117).

Given the definition Eq. (119) of the SO(4) ChernSimons term via the extension to $Y_{4}$, we can rewrite it in terms of field strength of $\mathrm{SU}(2)_{s}$ and $\mathrm{SU}(2)_{g}$ gauge fields $A^{s}$ and $a^{g}$, or, alternatively, their $\mathrm{SO}(3)$ representations, [119] obtaining

$$
\begin{aligned}
& \mathrm{CS}_{\mathrm{SO}(4)}\left[A^{s, g}, Y_{4}\right]+4 \mathrm{CS}_{g}\left[Y_{4}\right] \\
& =\mathrm{CS}_{\mathrm{SU}(2)}\left[A^{s}, Y_{4}\right]+\mathrm{CS}_{\mathrm{SU}(2)}\left[a^{g}, Y_{4}\right]+4 \mathrm{CS}_{g}\left[Y_{4}\right] \\
& =\frac{1}{2} \mathrm{CS}_{\mathrm{SO}(3)}\left[A^{s}, Y_{4}\right]+\frac{1}{2} \mathrm{CS}_{\mathrm{SO}(3)}\left[a^{g}, Y_{4}\right]+4 \mathrm{CS}_{g}\left[Y_{4}\right],
\end{aligned}
$$

where, as usual for an $\mathrm{SU}(2)$ gauge field $A$,

$$
\mathrm{CS}_{\mathrm{SU}(2)}\left[A, Y_{4}\right]=\frac{1}{4 \pi} \int_{Y_{4}} \operatorname{tr}_{\mathrm{SU}(2)} F \wedge F,
$$

and the trace is in the spin-1/2 representation. The halfinteger-level SO(3) Chern-Simons terms in Eq. (122) are not independent of $Y_{4}$ individually, but the full sum is independent of $Y_{4}$ [the integer-level $\mathrm{SU}(2)$ terms in Eq. (121) are also not individually independent of $Y_{4}$, since transition functions for $\mathrm{SU}(2)_{s}$ and $\mathrm{SU}(2)_{g}$ do not independently satisfy the cocycle condition]. It is instructive to check this statement without appealing to the APS theorem. To show that Eq. (122) is independent of $Y_{4}$, it suffices to check that it vanishes modulo $2 \pi$ when $Y_{4}$ has no boundary. Recalling that for an $\mathrm{SO}(n)$ gauge bundle on a closed manifold $Y_{4}$ the first Pontryagin number is given by [111]

$$
p_{1}=\frac{1}{2(2 \pi)^{2}} \int_{Y_{4}} \operatorname{tr}_{\mathrm{SO}(n)} F \wedge F
$$

and the signature of the manifold is [111]

$$
\sigma=-\frac{1}{24 \pi^{2}} \int_{Y_{4}} \operatorname{tr} R \wedge R
$$

we must show that

$$
p_{1}^{\mathrm{SO}(3)}\left[A^{s}, Y_{4}\right]+p_{1}^{\mathrm{SO}(3)}\left[a^{g}, Y_{4}\right]-\sigma\left[Y_{4}\right]=0 \quad(\bmod 4)
$$

for any closed $Y_{4}$. Now, for an $\mathrm{SO}(n)$ gauge bundle [44],

$$
p_{1}=\mathcal{P}\left(w_{2}\right)+2 w_{4} \quad(\bmod 4)
$$

where $w_{i}$ are the Stiefel-Whitney classes of the bundle and $\mathcal{P}: H^{2}\left(Z_{2}\right) \rightarrow H^{4}\left(Z_{4}\right)$ is the Pontryagin square operation, which satisfies $\mathcal{P}(a+b)=\mathcal{P}(a)+\mathcal{P}(b)+2 a \cup b$ (mod 4) (see Appendix D for details). Recalling that $w_{2}\left[A^{s}\right]+w_{2}\left[a^{g}\right]+w_{2}\left(T Y_{4}\right)=0$, and that for $\mathrm{SO}(3)$ bundles $w_{4}=0$, Eq. (126) reduces to

$$
\begin{aligned}
& p_{1}\left[A^{s}, Y_{4}\right]+p_{1}\left[a^{g}, Y_{4}\right]-\sigma\left[Y_{4}\right] \\
& =\int_{Y_{4}}\left\{2 \mathcal{P}\left(w_{2}\left[A^{s}\right]\right)+2 w_{2}\left[A^{s}\right] \cup w_{2}\left[T Y_{4}\right]+\mathcal{P}\left(w_{2}\left[T Y_{4}\right]\right)\right\} \\
& \quad-\sigma\left[Y_{4}\right],
\end{aligned}
$$

where all manipulations are modulo 4 . On an orientable four-manifold $Y_{4}$, for any $a \in H^{2}\left(Z_{2}\right), a \cup a=a \cup w_{2}\left[T Y_{4}\right]$ (see, e.g., Ref. [120], p. 132); furthermore, $\mathcal{P}(a)=a \cup a$ $(\bmod 2)$, so the first two terms on the rhs of Eq. (128) add to $0 \bmod 4$. The remaining statement, $\int_{Y_{4}} \mathcal{P}\left(w_{2}\left[T Y_{4}\right]\right)=\sigma$ $(\bmod 4)$, is also true [121].

\section{B. Gauging SO(5)}

We are now ready to discuss gauging of the full $\mathrm{SO}(5)$ global symmetry of $\mathrm{QCD}_{3}$. Given an $\mathrm{SO}(5)$ gauge bundle with connection $A^{5}$ on our three-manifold $M$, we attempt to lift it to $\mathrm{Sp}(4)=\operatorname{Spin}(5)$. The resulting transition functions may not satisfy the cocycle condition: the defect is $w_{2}\left[A^{5}\right]$. As before, we choose $\mathrm{SU}(2)_{g}$ transition functions so that the combination of $\mathrm{Sp}(4), \mathrm{SU}(2)_{g}$, and $\mathrm{Spin}(3)_{T M}$ transition functions satisfies the cocycle condition, i.e., $w_{2}\left[A^{5}\right]+w_{2}\left[a^{g}\right]+w_{2}[T M]=0(\bmod 2)$, with $w_{2}\left[a^{g}\right]$ being the second Stiefel-Whitney class of the $\mathrm{SO}(3)_{g}$ gauge bundle. Thinking of $\left[\mathrm{Sp}(4) \times \mathrm{SU}(2)_{g}\right] / Z_{2}$ as a subgroup of so(8), the (unregulated) action becomes 


$$
L_{\mathrm{QCD}_{3}}=\bar{\chi} \gamma^{\mu}\left(\partial_{\mu}+i \omega_{\mu}-i A_{\mu}^{5, g}\right) \chi
$$

with $A^{5, g}$ living in the so(8) Lie algebra, i.e., acting on spincolor $m$ and valley indices $v$ of $\chi$. We must now specify how to regulate the above action. We can no longer use PV regulators of opposite mass for the two valleys since this will break $\mathrm{SO}(5)$ symmetry. Instead, we use a common PV regulator for the so(8) vector $\chi$ and supplement the action by an so(8) Chern-Simons term,

$$
\begin{aligned}
S_{\mathrm{QCD}_{3}}= & \int_{M}\left[\bar{\chi} \gamma^{\mu}\left(\partial_{\mu}+i \omega_{\mu}-i A_{\mu}^{5, g}\right) \chi\right]_{\mathrm{PV},+} \\
& -\frac{i}{2} \mathrm{CS}_{\mathrm{SO}(8)}\left[A^{5, g}, Y_{4}\right]-4 i \mathrm{CS}_{g}\left[Y_{4}\right],
\end{aligned}
$$

with the Chern-Simons terms again defined by extending to a four-manifold $Y_{4}$, as before. When the $\mathrm{SO}(5)$ bundle reduces to an $\mathrm{SO}(3)_{s}$ bundle, Eq. (130) reduces to Eq. (118) as needed. But are the Chern-Simons terms in Eq. (130) independent of $Y_{4}$ for an arbitrary $\mathrm{SO}(5)$ bundle? We show that the answer is no: Eq. (130) is not well defined as a purely $(2+1) \mathrm{D}$ theory. However, we are able to define it as a surface of an $\mathrm{SO}(5)$-protected $(3+1) \mathrm{D}$ bosonic SPT phase.

First, we observe that if $A^{5, g}$ was an arbitary $\mathrm{SO}(8)$ bundle, Eq. (130) obviously would not define a purely $(2+1) \mathrm{D}$ theory, as the $\mathrm{SO}(8)$ level is fractional. In fact, physically, Eq. (130) is just the action of eight identical copies of a $(3+1) \mathrm{D}$ topological superconductor living on the space $Y_{4}$ and coupled to an $\mathrm{SO}(8)$ gauge field. The bulk of such a state has a nontrivial $\mathrm{SO}(8)$ response. Indeed, by the APS theorem Eq. (120), the partition function of Eq. (130) after integrating the fermions out becomes

$$
\exp \left(-S_{\mathrm{QCD}_{3}}\left[A^{5, g}\right]\right)=\left|Z_{\chi}\left[A^{5, g}\right]\right|(-1)^{\mathcal{J}\left[A^{5, g}, Y_{4}\right]},
$$

with $\left|Z_{\chi}\left[A^{5, g}\right]\right|$ the absolute value of the partition function for our eight $(2+1) \mathrm{D}$ Majorana fermions coupled to $A^{5, g}$, and $2 \mathcal{J}$ the index of $i D_{A^{5, g}}$ on $Y_{4}$. For closed $Y_{4}$ and a general $\mathrm{SO}(8)$ gauge field, $\mathcal{J}$ is not necessarily even, so $S_{\mathrm{QCD}_{3}}\left[A^{5, g}\right]$ depends on the extension to $Y_{4}$. However, our $A^{5, g}$ is not the most general $\mathrm{SO}(8)$ gauge field; rather, we are dealing with an $\left[\mathrm{Sp}(4) \times \mathrm{SU}(2)_{g} \times \operatorname{Spin}(3)_{T M}\right] /\left(Z_{2} \times Z_{2}\right)$ bundle: when this bundle is extended to the four-manifold $Y_{4}$, is Eq. (130) independent of the extension? [123] We rewrite,

$\frac{1}{2} \mathrm{CS}_{\mathrm{SO}(8)}\left[A^{5, g}, Y_{4}\right]=\frac{1}{2} \mathrm{CS}_{\mathrm{SO}(5)}\left[A^{5}, Y_{4}\right]+\frac{1}{2} \mathrm{CS}_{\mathrm{SO}(3)}\left[a^{g}, Y_{4}\right]$.

Thus, to check whether Eq. (130) is well defined as a $(2+1) \mathrm{D}$ theory, we must determine whether for closed $Y_{4}$
$p_{1}^{\mathrm{SO}(5)}\left[A^{5}, Y_{4}\right]+p_{1}^{\mathrm{SO}(3)}\left[a^{g}, Y_{4}\right]-\sigma\left[Y_{4}\right] \stackrel{?}{=} 0 \quad(\bmod 4)$.

We again use the identity Eq. (127), and $w_{2}\left[A^{5}\right]+w_{2}\left[a^{g}\right]+$ $w_{2}[T M]=0$. Repeating the manipulations below Eq. (126), we obtain

$p_{1}\left[A^{5}, Y_{4}\right]+p_{1}\left[a^{g}, Y_{4}\right]-\sigma\left[Y_{4}\right]=2 w_{4}\left[A^{5}, Y_{4}\right] \neq 0(\bmod 4)$

Thus, Eq. (130) generally depends on the extension to $Y_{4}$ : given two extensions to $Y_{4}$ and $\tilde{Y}_{4}$, we have

$$
\begin{aligned}
& \left.\exp \left\{-S_{\mathrm{QCD}_{3}}\left[A^{5, g}, \tilde{Y}_{4}\right]+S_{\mathrm{QCD}_{3}}\left[A^{5, g}, Y_{4}\right]\right)\right\} \\
& \quad=\exp \left(\pi i \int_{\tilde{Y}_{4} \cup \bar{Y}_{4}} w_{4}\left[A^{5}\right]\right)
\end{aligned}
$$

where the last integral is over the manifold obtained by gluing together $\tilde{Y}_{4}$ and $Y_{4}$ with reversed orientation. Crucially, the variation Eq. (135) depends only on the extension of the $\mathrm{SO}(5)$ bundle $A^{5}$, but not on the $\mathrm{SU}(2)_{g}$ bundle. Still, we cannot promote $\mathrm{SO}(5)$ to an on-site symmetry of a strictly $(2+1) \mathrm{D}$ theory. However, we can think of the theory Eq. (130) as the surface of an SO(5)protected $(3+1) \mathrm{D}$ SPT, as follows. Let $X_{4}$ be the physical $(3+1) \mathrm{D}$ manifold that our SPT phase lives on. There is an $\mathrm{SO}(5)$ gauge field $A^{5}$ on $X_{4}$. When $X_{4}$ has no boundary, we let the partition function be

$$
Z_{3+1}\left[A^{5}, X_{4}\right]=\exp \left(\pi i \int_{X_{4}} w_{4}\left[A^{5}\right]\right)
$$

When $X_{4}$ has a boundary, $\partial X_{4}=M$, Eq. (136) is not well defined [not gauge invariant under using different representatives of the cohomology class $\left.w_{4}\left[A^{5}\right] \in H^{4}\left(X_{4}, Z_{2}\right)\right]$. However, we can combine the surface action Eq. (130) with the bulk action Eq. (136), to obtain

$$
\begin{aligned}
S_{\mathrm{QCD}_{3}}^{\text {bulk+bound }}= & \int_{M}\left[\bar{\chi}_{v} \gamma^{\mu}\left(\partial_{\mu}+i \omega_{\mu}-i A^{5, g}\right) \chi_{v}\right]_{\mathrm{PV},+} \\
& -\frac{i}{2} \mathrm{CS}_{\mathrm{SO}(5)}\left[A^{5}, Y_{4}\right]-\frac{i}{2} \mathrm{CS}_{\mathrm{SO}(3)}\left[a^{g}, Y_{4}\right] \\
& -4 i \mathrm{CS}_{g}\left[Y_{4}\right]+\pi i \int_{X_{4} \cup \bar{Y}_{4}} w_{4}\left[A^{5}\right] .
\end{aligned}
$$

Again, the Chern-Simons terms in the second line of Eq. (137) are defined with the help of an extension to an auxiliary four-dimensional manifold $Y_{4}$. The term in the last line of Eq. (137) involves an integral over a manifold obtained by gluing $X_{4}$ and $Y_{4}$ with its orientation reversed. Every line in Eq. (137) is well defined; however, the second and third lines individually depend on the extension to $Y_{4}$. 
However, the $Y_{4}$ dependence of the second and third lines cancels, due to Eq. (135). Therefore, Eq. (137) is overall independent of $Y_{4}$. It does, however, depend on $A^{5}$ on the physical $(3+1) \mathrm{D}$ manifold $X_{4}$ and reduces to Eq. (136) when $X_{4}$ has no boundary. Therefore, Eq. (137) is a perfectly well-defined action of an $\mathrm{SO}(5)$-protected SPT phase on the $(3+1) \mathrm{D}$ manifold $X_{4}$ with boundary $M$ : only the background $\mathrm{SO}(5)$ gauge field $A^{5}$ lives in the bulk; the fields $a^{g}, \chi$ live on the surface. The independence of the variation Eq. (135) of $a^{g}$ is crucial for such a SPT interpretation to be possible.

Let us obtain some intuition for the bulk topological term Eq. (136). First, consider gauging just the $\mathrm{SO}(3)_{s} \times \mathrm{SO}(2)_{\text {VBS }}$ subgroup of $\mathrm{SO}(5)$; i.e., the $\mathrm{SO}(5)$ bundle is a direct sum of $\mathrm{SO}(3)_{s}$ and $\mathrm{SO}(2)_{\mathrm{VBS}}$ bundles. Label the corresponding gauge bundles as $A^{s}$ and $A^{\mathrm{VBS}}$. We have

$$
\begin{aligned}
2 w_{4}^{\mathrm{SO}(5)}\left[A^{5}\right]= & p_{1}\left[A^{5}\right]-\mathcal{P}\left(w_{2}\left[A^{5}\right]\right) \\
= & p_{1}^{\mathrm{SO}(3)}\left[A^{s}\right]+p_{1}^{\mathrm{SO}(2)}\left[A^{\mathrm{VBS}}\right] \\
& -\mathcal{P}\left(w_{2}\left[A^{s}\right]+w_{2}\left[A^{\mathrm{VBS}}\right]\right) \\
= & -2 w_{2}\left[A^{s}\right] \cup w_{2}\left[A^{\mathrm{VBS}}\right](\bmod 4),
\end{aligned}
$$

where we repeatedly use Eq. (127), together with the fact that $w_{2}$ and $p_{1}$ are additive under $\mathrm{SO}(n)$ bundle addition, and that $w_{4}=0$ for $\mathrm{SO}(n)$ with $n \leq 3$. So,

$w_{4}\left[A^{5}\right]=w_{2}\left[A^{s}\right] \cup w_{2}\left[A^{\mathrm{VBS}}\right]=w_{2}\left[A^{s}\right] \cup \frac{F^{\mathrm{VBS}}}{2 \pi}$,

where we use the fact that for an $\mathrm{SO}(2)$ gauge field, $w_{2}$ and the first Chern class $[F /(2 \pi)]$ coincide $\bmod 2: w_{2}=$ $[F /(2 \pi)](\bmod 2)[124]$. What is the physical interpretation of Eq. (139)? Imagine we take our bulk manifold to be $S^{2} \times \Sigma$, with $\Sigma$ an arbitrary two-dimensional surface. Place flux $2 \pi$ of $F^{\mathrm{VBS}}$ through $S^{2}$. Then the partition function Eq. (136) is $Z=\exp \left(\pi i \int_{\Sigma} w_{2}\left[A^{s}\right]\right)$. This is precisely the partition function of a $\mathrm{SO}(3)_{s}$-protected $(1+1) \mathrm{D}$ Haldane phase on $\Sigma$ [125]. We know that the Haldane phase on a spatial interval $I=[0,1]$ has dangling spin $1 / 2$ 's at the boundary. So, we can guess that if we consider the theory on the spatial manifold $S^{2} \times I$, then there are dangling spin $1 / 2$ 's at the two ends of $I$-i.e., at the locations of $\mathrm{SO}(2)_{\text {VBS }}$ monopoles. We, therefore, conclude that the topological term Eq. (139) makes monopoles of $\mathrm{SO}(2)_{\mathrm{VBS}}$ transform in the spin- $1 / 2$ representation of $\mathrm{SO}(3)_{s}$. This is precisely the conclusion reached by less formal methods in Sec. VIC.

We can further focus on just the easy-plane subgroup $\mathrm{SO}(2)_{s}$ of $\mathrm{SO}(3)_{s}$, then Eq. (139) reduces to

$$
S=\pi i \int_{X_{4}} w_{4}\left[A^{5}\right]=\pi i \int_{X_{4}} \frac{F^{s}}{2 \pi} \cup \frac{F^{\mathrm{VBS}}}{2 \pi} .
$$

This is precisely the mutual $\theta=\pi$ term for $\mathrm{U}(1)_{s} \times \mathrm{U}(1)_{\text {VBS }}$. Note that to protect this $\theta$ value from shifting, one needs to rely on some discrete symmetry, such as spin-flip symmetry or time reversal.

What if we restrict ourselves to an $\mathrm{SO}(4)$ subgroup of $\mathrm{SO}(5)$ ? Let us write $\mathrm{SO}(4)=\left[\mathrm{SU}(2)_{L} \times \mathrm{SU}(2)_{R}\right] / Z_{2}$. Label associated $\mathrm{SO}(3)_{L}$ and $\mathrm{SO}(3)_{R}$ gauge fields as $A^{L}$ and $A^{R}$. We have $w_{2}\left[A^{L}\right]=w_{2}\left[A^{R}\right]=w_{2}\left[A^{5}\right]$. Now,

$$
\begin{aligned}
2 w_{4}\left[A^{5}\right] & =p_{1}\left[A^{5}\right]-\mathcal{P}\left(w_{2}\left[A^{5}\right]\right) \\
& =\frac{1}{2} p_{1}^{\mathrm{SO}(3)}\left[A^{L}\right]+\frac{1}{2} p_{1}^{\mathrm{SO}(3)}\left[A^{R}\right]-\mathcal{P}\left(w_{2}\left[A^{R}\right]\right) \\
& =\frac{1}{2} p_{1}\left[A^{L}\right]-\frac{1}{2} p_{1}\left[A^{R}\right] \quad(\bmod 4) .
\end{aligned}
$$

Therefore, Eq. (136) reduces to

$$
\begin{aligned}
S & =\frac{1}{4} \mathrm{CS}_{\mathrm{SO}(3)}\left[A^{L}, X_{4}\right]-\frac{1}{4} \mathrm{CS}_{\mathrm{SO}(3)}\left[A^{R}, X_{4}\right] \\
& =\frac{1}{2} \mathrm{CS}_{\mathrm{SU}(2)}\left[A^{L}, X_{4}\right]-\frac{1}{2} \mathrm{CS}_{\mathrm{SU}(2)}\left[A^{R}, X_{4}\right] .
\end{aligned}
$$

In $\mathrm{SU}(2)$ terminology Eq. (112), this corresponds to opposite $\theta$ angles for $\mathrm{SU}(2)_{L}$ and $\mathrm{SU}(2)_{R}: \theta_{L}=-\theta_{R}=\pi$. Again, discrete symmetries, e.g., $Z_{2}=\mathrm{O}(4) / \mathrm{SO}(4)$, which maps $R \leftrightarrow L$, and time reversal, are required to fix these $\theta$ angles from flowing.

\section{Bulk parton construction for boson SPT with $\mathrm{SO}(5) \times Z_{2}^{T}$ symmetry: Formal derivation}

In this section, we reconsider from a more formal standpoint the parton construction of the $(3+1) \mathrm{D} \mathrm{SO}(5) \times Z_{2}^{T}$ boson SPT presented at the end of Sec. VI C. We show that this construction precisely recovers the bulk SPT "discrete $\theta$-angle" response in Eq. (136). This provides a more physical motivation for our $(3+1) \mathrm{D}$ bulk "completion" of $\mathrm{QCD}_{3}$ in Eq. (137).

As in Sec. VIC, we begin by considering a $(3+1) \mathrm{D}$ "topological superconductor" of fermions with $\mathrm{SO}(8) \times Z_{2}^{T}$ symmetry. We will then gauge an $S U(2)$ subgroup and show that the result is precisely the boson SPT of interest. As explained in Sec. VIC, we represent the $\mathrm{SO}(8) \times Z_{2}^{T}$ symmetric topological superconductor by eight massive Majorana fermions with an inverted mass, Eq. (110). Let us consider the partition function on a manifold $X_{4}$ in the presence of a background $\mathrm{SO}(8)$ gauge bundle $A^{8}$. We restrict ourselves to closed oriented manifolds. The partition function takes the form (see Appendix E)

$$
Z_{\mathrm{TSC}}\left[A^{8}\right]=\left|Z_{\mathrm{TSC}}\right| e^{i \pi\left\{\left(p_{1}\left[A^{8}\right] / 2\right)-(\sigma / 2)\right\}},
$$

with the $p_{1}, \sigma$ given by Eqs. (124) and (125). Further, by the Atiyah-Singer index theorem Eq. (120), we have 


$$
\frac{p_{1}\left[A^{8}\right]}{2}-\frac{\sigma}{2}=\mathcal{J} \in Z
$$

Here, $2 \mathcal{J}$ is the index of the $(3+1) \mathrm{D}$ massless Dirac operator on $X_{4}$. This implies that the partition function in Eq. (143) is real (but not necessarily positive), as required for a time-reversal invariant SPT state.

Note that on a spin manifold $\left\{\left[p_{1}\left(A^{8}\right)\right] / 2\right\}$ is itself an integer (and so is $\sigma / 2$ ). This follows because by Eq. (127), $p_{1}\left[A^{8}\right]=w_{2}\left[A^{8}\right] \cup w_{2}\left[A^{8}\right](\bmod 2)=w_{2}\left[A^{8}\right] \cup w_{2}\left[T X_{4}\right]=0$ $(\bmod 2)$. However, as we eventually want to describe a boson SPT, it is important to be able to formulate the theory on a nonspin manifold. As usual, since the fermions see transition functions in $\left[\mathrm{SO}(8) \times \operatorname{Spin}(4)_{T X_{4}}\right] / Z_{2}$, we require only these transition functions to satisfy the cocycle condition. Then, though $\left\{\left[p_{1}\left(A^{8}\right)\right] / 2\right\}$ and $\sigma / 2$ are not separately integers, their sum is.

Now, to construct the boson SPT phase, we gauge the $\mathrm{SU}(2)_{g}$ subgroup of $\mathrm{SO}(8)$, as in Eq. (111). We further couple the system to a background $\mathrm{SO}(5)$ gauge field $A^{5}$. As we discuss in Sec. VIC, the partons $\chi$ transform as $\mathrm{Sp}(4)$ spinors under $\mathrm{SO}(5)$, so they see a combined $\left[\mathrm{Sp}(4) \times \mathrm{SU}(2)_{g}\right] / Z_{2}$ gauge field. Together with the $\operatorname{Spin}(4)_{T X_{4}}$ transition functions, this yields overall transition functions in $\left[\mathrm{Sp}(4) \times \mathrm{SU}(2) \times \operatorname{Spin}(4)_{T X_{4}}\right] /\left(Z_{2} \times Z_{2}\right)$. This gives the by now familiar condition

$$
w_{2}\left[A^{5}\right]+w_{2}\left[a_{g}\right]+w_{2}\left[T X_{4}\right]=0 \quad(\bmod 2) .
$$

For such an $\mathrm{SO}(8)$ bundle, we have

$$
p_{1}\left[A^{8}\right]=p_{1}\left[A^{5}\right]+p_{1}\left[a_{g}\right] .
$$

Thus, applying Eq. (134), we obtain

$$
Z_{\mathrm{TSC}}\left[A^{5}, a_{g}\right]=\left|Z_{\mathrm{TSC}}\right| e^{i \pi \int_{X_{4}} w_{4}\left[A^{5}\right]} .
$$

Thus far we have treated $a_{g}$ as a background gauge field. Now we make it dynamical; i.e., we integrate the partition function over $a_{g}$. There is no topological term for $a_{g}$ and its dynamics will be governed by the usual Yang-Mills action, which is expected to confine all fields charged under $a_{g}$ leaving behind a trivial gapped vacuum. The resulting theory has a partition function with a phase given precisely by $e^{i \pi \int_{X_{4}} w_{4}\left[A^{5}\right]}$. Further, it can be formulated on a nonspin manifold with only a background $\mathrm{SO}(5)$ gauge field. The local operators are bosonic and transform nonprojectively under $\mathrm{SO}(5)$. Thus, we have constructed the desired boson SPT with $\mathrm{SO}(5) \times Z_{2}^{T}$ symmetry, and the partition function matches Eq. (136) proposed in Sec. VII B based on consistency arguments.

\section{Chiral spin liquid}

We note that if we break time reversal in $\mathrm{QCD}_{3}$ with an $\mathrm{Sp}(4)$-preserving mass term,

$$
\delta L=m \bar{\chi} \chi,
$$

and make the mass $m$ large enough (compared say to the gauge coupling), we drive the system into a topologically ordered phase. Integrating the gapped $\chi$ 's in Eq. (137) out,

$$
\begin{aligned}
S_{\mathrm{QCD}_{3}}^{\text {bulkound }}= & \operatorname{sgn}(m)\left[\frac{i}{2} \mathrm{CS}_{\mathrm{SO}(5)}\left[A^{5}, Y_{4}\right]+i \mathrm{CS}_{\mathrm{SU}(2)}\left[a^{g}, Y_{4}\right]\right. \\
& \left.+4 i \mathrm{CS}_{g}\left[Y_{4}\right]\right]+\pi i \int_{X_{4} \cup \bar{Y}_{4}} w_{4}\left[A^{5}\right] .
\end{aligned}
$$

Without loss of generality, choose $m>0$. By looking at the action for $a_{g}$, we see that we get a $(2+1) \mathrm{D} \mathrm{SU}(2)_{1}$ topological order, which is just a semion state $\{1, s\}$. The semion $s$ is just the Majorana $\chi$. The chiral central charge $c=2-1$, with $2=4 \times 1 / 2$ coming from the gravitational Chern-Simons term and -1 from integrating $a_{g}$ out. The semion $\chi$ transforms projectively under the $\mathrm{SO}(5)$ symmetry - as an $\mathrm{Sp}(4)$ spinor. In particular, it carries spin $1 / 2$ under $\mathrm{SO}(3)_{s}$. Further, the $\mathrm{SO}(5)$ response is given by a Chern-Simons term with level $k=1 / 2$, so the level of Chern-Simons response to $\mathrm{SO}(3)_{s}$ gauge field is also $1 / 2$. We see that this state has all the properties of a chiral spin liquid [94].

Now, it would be a little surprising if the chiral spin liquid was in the vicinity of the deconfined quantum critical point. If we consider the $\mathrm{NCCP}^{1}$ formulation Eq. (1), then one operator with the same quantum numbers as $\bar{\chi} \chi$ is $\epsilon_{\mu \nu \lambda} \partial_{\rho} f_{\rho \mu}^{b} f_{\nu \lambda}^{b}$, with $f_{\mu \nu}^{b}=\partial_{\mu} b_{\nu}-\partial_{\nu} b_{\mu}$. Given the number of derivatives, one would naively expect this term to be irrelevant in the $\mathrm{NCCP}^{1}$ model. If $\mathrm{NCCP}^{1}$ and $\mathrm{QCD}_{3}$ indeed share the same fixed point, this would then imply that $\bar{\chi} \chi$ is irrelevant at the $\mathrm{QCD}_{3}$ fixed point-an unexpected, but not impossible scenario. It would be interesting to determine the scaling dimension of this operator numerically at the deconfined critical point. In the lattice magnet, it corresponds to the imaginary part of the plaquette ring exchange:

$$
\begin{aligned}
\bar{\chi} \chi \sim & \frac{-i}{2}\left(P_{i+\hat{y}, i+\hat{x}+\hat{y}} P_{i+\hat{x}+\hat{y}, i+\hat{x}} P_{i+\hat{x}, i}-\text { H.c. }\right) \\
\sim & \mathbf{S}_{i+\hat{x}+\hat{y}} \cdot\left(\mathbf{S}_{i} \times \mathbf{S}_{i+\hat{x}}\right)+\mathbf{S}_{i+\hat{y}} \cdot\left(\mathbf{S}_{i} \times \mathbf{S}_{i+\hat{x}}\right) \\
& +\mathbf{S}_{i+\hat{y}} \cdot\left(\mathbf{S}_{i+\hat{x}} \times \mathbf{S}_{i+\hat{x}+\hat{y}}\right)+\mathbf{S}_{i+\hat{y}} \cdot\left(\mathbf{S}_{i} \times \mathbf{S}_{i+\hat{x}+\hat{y}}\right),
\end{aligned}
$$

where $P_{i j}=2 \mathbf{S}_{i} \cdot \mathbf{S}_{j}+1 / 2$ is the exchange operator.

\section{DISCUSSION AND IMPLICATIONS OF THE DUALITIES}

The most fundamental question about both the SU(2)invariant and the easy-plane $\mathrm{NCCP}^{1}$ theories is whether 
they describe CFTs in the IR. We do not tackle this question head-on in this paper. We first discuss what follows if the dualities are assumed to hold in their strong forms in the IR. As mentioned in the Introduction, the various theories could fail to flow to nontrivial fixed points. In this scenario the dualities may still be relevant to the "quasiuniversal" physics up to a large length scale. We discuss these issues, and what is known about the IR fate of the deconfined critical transitions, in Sec. IX.

\section{A. Deconfined criticality}

Many consequences of the emergent $\mathrm{SO}(5)$ symmetry have been explored numerically in Ref. [15]. In Sec. IV E, we discuss an additional consequence for the phase diagram in the presence of perturbations that break $\mathrm{SO}(5)$. This will be interesting to explore in future numerical work.

We argue that the proposed duality web provides an explanation of this emergent $\mathrm{SO}(5)$ symmetry, despite the fact that the $\mathrm{SO}(5)$ symmetry is not manifest in any single member of the duality web. In particular, the proposed selfduality of the $\mathrm{SU}(2)$-invariant $\mathrm{NCCP}^{1}$ model immediately implies emergent $\mathrm{SO}(5)$ symmetry in the IR. We discuss other numerical tests of the fermionic versions of this theory separately below.

We also discuss the continuum $N_{f}=2 \mathrm{QCD}_{3}$ theory which has manifest $\mathrm{SO}(5)$ symmetry and which shares the same anomaly as the putative deconfined critical point, and may possibly flow to it in the IR. The IR fate of the $\mathrm{QCD}_{3}$ theory is not currently known and is a good target for future numerical work. It will be particularly interesting to see if it shares the (quasi)universal power-law correlations seen in other models equivalent to the $\mathrm{SU}(2)$-invariant $\mathrm{NCCP}^{1}$.

For the easy-plane model, direct numerical simulations of quantum magnets find a first-order transition. As we have emphasized several times, the nature of the transition in this model is worth revisiting. We have seen that this model is dual to a version of fermionic $N_{f}=2 \mathrm{QED}_{3}$ with $\mathrm{U}(1) \times \mathrm{U}(1)$ symmetry. For fermionic $\mathrm{QED}_{3}$ with $\mathrm{SU}(2)$ flavor symmetry, there is some recent evidence that the theory is conformal in the IR [45]. Further the results do not seem to be sensitive to whether the lattice regulator employed actually preserves full $\mathrm{SU}(2)$ flavor symmetry or whether it has only U(1) flavor symmetry. In light of all this, more numerical studies of the models in the easy-plane duality web are clearly called for.

The strongest form of the duality web of these theories asserts that all these theories flow in the IR to the same $\mathrm{O}(4) \times Z_{2}^{T}$-invariant CFT. Below, we describe some implications of the enhanced symmetry expected in such a putative critical theory.

If an $\mathrm{O}(4) \times Z_{2}^{T}$-symmetric fixed point does exist, then for the easy-plane $\mathrm{NCCP}^{1}$ to flow to it in the IR it must be that perturbations that break the symmetry to $\left\{\mathrm{U}(1) \times\left[U(1) \rtimes Z_{2}\right]\right\} \times Z_{2 T}$ are irrelevant. As we discuss in Sec. III C, the simplest such perturbation is a Néel-VBS anisotropy which lies in the $(2,2)$ representation of $\mathrm{SO}(4)$ (the quadrupled monopole operator $\Phi_{2}^{4}+\Phi_{2}^{* 4}$ pertinent to the lattice magnet lies in the same representation). Thus, for enlarged $\mathrm{O}(4)$ symmetry we need the scaling dimension $\Delta_{(2,2)}>3$ at the $\mathrm{O}(4)$ fixed point.

If an $\mathrm{O}(4) \times Z_{2}^{T}$-symmetric fixed point exists, and the strongest form of the duality web holds, then a square lattice spin-1/2 quantum magnet with $X Y$ symmetry can show a direct continuous Néel-VBS transition with enlarged $\mathrm{O}(4)$ symmetry.

An alternate possibility is that the $\mathrm{O}(4) \times Z_{2}^{T}$ CFT exists and that $\mathrm{SU}(2)$ flavor symmetric $\mathrm{QED}_{3}$ flows to it, but the $\mathrm{U}(1) \times \mathrm{U}(1)$ theories (easy-plane $\mathrm{NCCP}^{1}$ and $\mathrm{QED}_{3}$ with the same symmetry) do not flow to that fixed point. This scenario can be tested by numerical simulations of the $\mathrm{SU}(2)$-symmetric $\mathrm{QED}_{3}$ theory. We detail below how to test for emergent $\mathrm{O}(4)$ symmetry. Should such a fixed point be found, it will be interesting to calculate the scaling dimension of operators transforming under the $(2,2)$ representation to test for relevance.

Finally, it is possible that an $\mathrm{O}(4) \times Z_{2}^{T}$ fixed point of the kind we describe does not exist in the first place. Therefore, we next turn to the fermionic theories where this question is best addressed numerically.

Useful analytical insights will also come from conformal bootstrap [25,26,127-129]. Note also that the duality webs open up the possibility of analytical results for deconfined critical points using large $N$ in the fermionic language [130-132].

\section{B. QED $_{3}$ and QED-GN}

The strong self-duality for $\mathrm{SU}(2)$ flavor symmetric $\mathrm{QED}_{3}$ implies an emergent $\mathrm{O}(4)$ symmetry which leads to simple testable predictions.

The fermion bilinears $\bar{\psi} \sigma \psi$ are expected to be scaling fields, with dimension smaller than their engineering dimension of two, transforming in the $(1,1)$ representation of $\mathrm{SO}(4)$. $\mathrm{O}(4)$ symmetry relates them to strength-2 monopole operators in the $\mathrm{QED}_{3}$ theory, so calculating correlations of monopole operators will allow interesting tests of the emergent symmetry. Presumably this requires some modifications of existing numerical calculations of correlators in the $\mathrm{QED}_{3}$ theory. We therefore also describe several tests using more ordinary correlators.

It should be fruitful to focus on correlations of the conserved $\mathrm{SO}(4)$ currents. The operator $\bar{\psi} \gamma^{0} \sigma \psi$ [the time component of one of the $\mathrm{SU}(2)$ currents] was already studied in Ref. [45] and shown to have the expected scaling dimension 2. One of the three currents of the other $\mathrm{SU}(2)$ is a simple operator in $\mathrm{QED}_{3}$ : this is the gauge flux $\epsilon_{\mu \nu \lambda} \partial_{\nu} a_{\lambda}$. Therefore, its time component, the magnetic flux, is related by symmetry to $\bar{\psi} \gamma^{0} \sigma \psi$. Right at the critical point these operators should have scaling dimension 2; this follows from their conservation and is not a test of the symmetry rotating them. A simple consequence of emergent $\mathrm{O}(4)$ is 
that the universal amplitudes of the two point functions should also be the same for these different currents. Specifically, if we compare the correlators of the SU(2) currents with the correlators of $[1 /(2 \pi)] \epsilon_{\mu \nu \lambda} \partial_{\nu} a_{\lambda}$, they should have the same universal amplitude in addition to the same scaling dimension.

A more dramatic consequence arises if we perturb the critical point by turning on either a nonzero temperature $T$ or a fermion mass $m$, both of which preserve $\mathrm{SO}(4)$. Then the current correlations will involve a nontrivial universal scaling function:

$$
|k| F\left(\frac{m}{T}, \frac{\omega}{|k|}, \frac{|k|}{m}\right) .
$$

Now $\mathrm{SO}(4)$ symmetry predicts that this scaling function is identical for the SU(2) current and for the 3-flux of the gauge field. It will be very interesting to test this. For instance, the SU(2) spin susceptibility should be described by the same crossover function as the diamagnetic susceptibility of the gauge field, and likewise the SU(2) phase stiffness should be described by the same crossover function as the Meissner stiffness of the gauge field.

Finally, a representative of the important $(2,2)$ operator will be given by, e.g., $2\left(\bar{\psi} \sigma^{z} \psi\right)^{2}-\left(\bar{\psi} \sigma^{x} \psi\right)^{2}-\left(\bar{\psi} \sigma^{y} \psi\right)^{2}$ [we are assuming that the other $\mathrm{SO}(4)$ representations contributing to this operator are less relevant, as expected from the discussion in Sec. III C]. If O(4) symmetry is established numerically, then the irrelevance of this $\mathrm{O}(4)$-breaking perturbation can be tested.

For the $\mathrm{QED}_{3}-\mathrm{GN}$ model, the first issue that should be addressed numerically is whether the transition is second order at all (the duality with the $\mathrm{NCCP}^{1}$ model suggests there should be critical behavior at least up to a large length scale). Should such a second-order transition be found, a number of its properties can be predicted using our results.

First, if we measure $\phi$ correlations at this fixed point, we are measuring correlations of the $\mathrm{SO}(5)$ vector. They can therefore be compared with the Néel and VBS correlation functions known from $\mathrm{NCCP}^{1}$ simulations. Second, the $\phi^{2}$ operator takes us to the $\mathrm{QED}_{3}$ or easy-plane $\mathrm{NCCP}^{1}$ fixed point. We know that with $\mathrm{SO}(5)$ this is in the same representation as the operator that tunes through the transition in the $\mathrm{NCCP}^{1}$ theory (a component of $X^{(2)}$ in the notation of Sec. IV D). Hence, the $\phi^{2}$ scaling dimension can be compared with results for $\nu$ at the $\mathrm{SU}(2)$-symmetric deconfined critical point.

More interestingly, the fermion bilinear $\bar{\psi} \sigma^{z} \psi$ also corresponds to an element of $X^{(2)}$. Thus, the vector $\bar{\psi} \sigma \psi$ should have the same correlations as $\phi^{2}$ at the $\mathrm{QED}_{3}$ - GN fixed point (modulo subleading contributions) if there is full $\mathrm{SO}(5)$ symmetry. This last statement is particularly interesting as it does not involve comparing with a different theory - both quantities are calculated in the same simulation.

\section{Comparison between the $N=2 \mathrm{QED}_{3}$, bilayer honeycomb lattice model, and easy-plane spin models}

For the putative $\mathrm{O}(4)$ fixed point, there are (at least) three lattice model realizations that can be (and have been) studied numerically: $N=2$ lattice $\mathrm{QED}_{3}$, spin models that realize the easy-plane deconfined transition (if a model with a second-order transition exists), and the bilayer honeycomb lattice interacting fermion model, studied in Refs. [46,47], that realizes the transition between a trivial and SPT boson insulator with explicit $\mathrm{SO}(4)$ symmetry. The critical exponents measured in different models should be related to each other, which we discuss below.

The $N=2 \mathrm{QED}_{3}$ was treated as a stable CFT in Ref. [45], so there is no correlation length critical exponent. But there is still the anomalous dimensions associated with the mass operators $M_{z}=\bar{\psi}_{1} \psi_{1}-\bar{\psi}_{2} \psi_{2}, \quad M_{0}=$ $\bar{\psi}_{1} \psi_{1}+\bar{\psi}_{2} \psi_{2}$,

$$
\left\langle M_{z}(0) M_{z}(r)\right\rangle \sim \frac{1}{r^{1+\eta_{\bar{\psi} \sigma^{2} \psi}}}, \quad\left\langle M_{0}(0) M_{0}(r)\right\rangle \sim \frac{1}{r^{1+\eta_{\bar{\mu} \psi}}} .
$$

According to Ref. [45], $\eta_{\bar{\psi} \sigma^{z} \psi} \sim 1$.0. To our knowledge, a careful study of $\eta_{\bar{\psi} \psi}$ has not been performed in numerical simulations of $\mathrm{QED}_{3}$-we hope that future simulations will also address this exponent.

The bilayer honeycomb lattice model describes a bosonic transition, which may potentially also be described by the $N=2 \mathrm{QED}_{3}$ [133]. The tuning parameter for this transition corresponds to the fermion mass $m\left(\bar{\psi}_{1} \psi_{1}+\bar{\psi}_{2} \psi_{2}\right)$ in the field theory. There is a correlation length exponent $\nu_{\mathrm{bh}}$ defined as

$$
\xi \sim m^{-\nu_{\mathrm{bh}}} \sim\left(J-J_{c}\right)^{-\nu_{\mathrm{bh}}},
$$

where $J$ is the interaction on the lattice that is tuned to the critical point. The $\mathrm{O}(4)$ order parameter $n_{a}$ has an anomalous dimension $\eta_{\mathrm{bh}}$ :

$$
\left\langle n_{a}(0) n_{a}(r)\right\rangle \sim \frac{1}{r^{1+\eta_{\mathrm{bh}}}} .
$$

The easy-plane spin models have three different exponents, $\eta_{x y}$ (the same as $\eta_{v b s}$ ), $\eta_{z}$, and $\nu_{j q}$ :

$$
\begin{aligned}
\xi & \sim\left(Q-Q_{c}\right)^{-\nu_{j q},} \\
\left\langle S^{x}(0) S^{x}(r)\right\rangle & \sim \frac{(-1)^{r}}{r^{1+\eta_{x y}}}, \\
\left\langle S^{z}(0) S^{z}(r)\right\rangle & \sim \frac{(-1)^{r}}{r^{1+\eta_{z}}},
\end{aligned}
$$


where $Q$ is a tuning parameter for the transition. If the strong duality holds, we have the following relations:

$$
\begin{aligned}
3-\frac{1}{\nu_{j q}} & =\frac{1+\eta_{\bar{\psi} \sigma^{z} \psi}}{2}, \\
\eta_{z} & =\eta_{\bar{\psi} \psi}, \quad \eta_{x y}=\eta_{\mathrm{bh}}, \\
3-\frac{1}{\nu_{\mathrm{bh}}} & =\frac{1+\eta_{z}}{2} .
\end{aligned}
$$

\section{CRITICAL AND PSEUDOCRITICAL POINTS}

It is not yet certain whether the $\mathrm{SU}(2)$-symmetric $\mathrm{NCCP}^{1}$ model has a true critical point, or whether it instead shows pseudocritical behavior with a very large but finite correlation length. Here, we review what is currently known from simulations, and clarify what the latter possibility would mean for the dualities we present here. We also briefly discuss the easy-plane case.

Various lattice models that show a phase transition "in the NCCP ${ }^{1}$ universality class" have been studied numerically $[6-22,134]$. The basic feature of these simulations is that the correlation length $\xi$ appears to diverge as the critical point is approached, certainly becoming larger than numerically accessible system sizes (up to 640 lattice spacings in the model of Ref. [14]). At these length scales the standard signs of first-order behavior, e.g., doublepeaked probability distributions, are absent. The qualitative features of the transition are as expected from the theory of deconfined criticality, [139] and finite-size estimates of critical exponents are roughly consistent between different lattice models.

These features are consistent with a continuous transition (which much recent work assumes). However, it was noted some time ago that various naively "universal" quantities instead drift with system size, leading to controversy about whether the transition was ultimately continuous or first order [9-13,17]. Reference [14] argued that these drifts are not merely conventional finite-size corrections to CFT scaling behavior, since making this assumption leads to unphysical negative values for the anomalous dimensions at large sizes, and suggested two possible scenarios for reconciling the various numerical results. One scenario is that the $\mathrm{NCCP}^{1}$ model shows a continuous transition, but with unconventional finite-size scaling behavior due to a dangerously irrelevant variable [140] (see also Refs. [24,137]). The second scenario is that $\mathrm{NCCP}^{1}$ shows a first-order transition $[12,13,17]$ which is rendered anomalously weak by a quasiuniversal mechanism [14], which we discuss below.

Further complicating the issue, it was found numerically that critical fluctuations at the deconfined transition are $\mathrm{SO}(5)$ symmetric to a high level of precision [15]. Level degeneracies found in the JQ model [141] also support this enhanced symmetry (the approximate equality of Néel and
VBS scaling dimensions had been noticed earlier by Sandvik [142]). At first sight $\mathrm{SO}(5)$ symmetry seems to be strong evidence that the critical $\mathrm{NCCP}^{1}$ model flows to an $\mathrm{SO}(5)$-invariant CFT. However, subsequent investigations [25,26] of $\mathrm{SO}(5)$-symmetric CFTs using the conformal bootstrap [127,128] did not find a sufficiently stable [143] CFT in the expected region of parameter space. The bootstrap shows that any sufficiently stable $\mathrm{SO}(5)$-invariant CFT must have a larger anomalous dimension for the $\mathrm{SO}(5)$ vector than is expected from simulations of deconfined criticality $[25,26]$. In view of this, it makes sense to revisit the weakly-first-order scenario with $\mathrm{SO}(5)$ symmetry in mind.

At first sight a first-order transition with $\xi \gg 1$ is implausible because of a fine-tuning problem. If a theory has no nontrivial stable fixed point, the obvious way to get a large $\xi$ is to fine-tune it close to an unstable fixed point [144]. Since this mechanism relies on fine-tuning, it is unlikely to be the explanation for the apparent critical behavior at the deconfined critical point (DCP), which seems to be generic. However there is an alternative generic mechanism for pseudocritical behavior with very large $\xi$ [145-147]. In this scenario, the large $\xi$ can be understood in terms of a fixed point which exists slightly outside the physical parameter space of the model-for example, at slightly smaller spatial dimension $d_{c}$. The structure of the RG flows close to $d_{c}$ implies an exponentially large correlation length for $d \gtrsim d_{c}$. This mechanism depends on an accident in the universal structure of the RG flows, but it does not require fine-tuning of a given microscopic Hamiltonian. Additionally, this scenario is plausible for the $\mathrm{NCCP}^{1}$ model (and indeed $\mathrm{NCCP}^{n-1}$ for nearby values of $n$ ), given what is known about the $d$-dimensional $\mathrm{NCCP}^{n-1}$ model in various limits [14].

The basic mechanism is the annihilation of a stable and an unstable fixed point as a parameter $\tau$ is varied. Here, $\tau$ is a quantity that does not flow under RG, such as the spatial dimension (in the case of $\mathrm{NCCP}^{1}$ ) or the rank of a symmetry group. Quite generally, close to $\tau_{c}$ the RG equation for the coupling $\lambda$ which is becoming marginal looks like

$$
\frac{d \lambda}{d \ln L}=a\left(\tau_{c}-\tau\right)-\lambda^{2},
$$

where $a$ and $\tau_{c}$ are universal constants and $a>0$. For $\tau<\tau_{c}$, both fixed points exist, and for $\tau>\tau_{c}$, neither do. But for $\tau \gtrsim \tau_{c}$ the RG flows become very slow close to $\lambda=0$ : the long $\mathrm{RG}$ time required to traverse the "pseudocritical" region corresponds to a large length scale $\xi \sim \ell_{0} \exp \left[\pi / \sqrt{a\left(\tau-\tau_{c}\right)}\right]$, where $\ell_{0}$ is nonuniversal. The large amount of RG time spent near $\lambda=0$ implies that the properties of the pseudocritical regime are quasiuniversal in the limit of small $\tau-\tau_{c}$. 
In more detail, this is because (in the formal limit of small $\tau-\tau_{c}$ ) the subleading RG couplings $g_{i}$ have time to flow to well-defined pseudocritical values, independent of their bare values in a given microscopic model. (The relevant coupling that drives the transition is zero since we consider the theory in the critical plane.) The RG flow is attracted to a quasiuniversal trajectory through coupling constant space, given by setting $g_{i}=0$ up to corrections that are exponentially small in $1 / \sqrt{\tau-\tau_{c}}$. A key point is that quasiuniversality holds to exponentially good precision in $1 / \sqrt{\tau-\tau_{c}}$, despite the fact that the flow of $\lambda$ during a stretch of RG time of order $\ln \xi$ is larger than this [148]. This flow of $\lambda$ will lead to quasiuinversal drifts in, e.g., effective exponents.

The $Q$-state Potts model in 2D provides an example of this phenomenon with $\tau=Q$ [145-147,150-153] (in this context $\lambda$ was originally thought of as a fugacity for Potts vacancies [145]). For $Q<4$, both a critical and a tricritical point exist, and they merge at $Q=4$. For $Q \gtrsim 4$, the Potts transition is very weakly first order. A priori the above picture applies only for $(Q-4) \ll 1$, but empirically it is found that the transition remains weakly first order at least for $Q=5,6,7$, where $\xi \simeq 2512,159,48$, respectively, on the square lattice [153]. This mechanism for generating a small mass scale has also been discussed in the context of 4D QCD [154-156], with $\tau=-N_{f} / N_{c}$. (Fixed point annihilation phenomena have also been discussed in $\mathrm{QED}_{3}$ [156-158], and in a Landau-Ginsburg theory obtained from $\mathrm{NCCP}^{n-1}$ by condensing the monopole $[159,160]$.) In the $\mathrm{NCCP}^{n-1}$ model, it is plausible that there is a range of $n$ where the transition is weakly first order but can be rendered continuous by slightly decreasing the spatial dimension.

It should be noted that the choice of deformation parameter $\tau$ is not unique; for example, in the weaklyfirst-order regime of the Potts model, the transition can be made continuous by reducing either $Q$ or $d$ (and in $\mathrm{NCCP}^{n-1}$ we can certainly render the transition continuous by a large enough increase in $n$ ). Alternately, one may consider the theory only at the physical value of $\tau$, and attribute the pseudocritical behavior to proximity to the nonunitary fixed points at $\lambda= \pm i \sqrt{a\left(\tau-\tau_{c}\right)}$.

A possible explanation for the various numerical results for the deconfined transition is that there is a pseudocritical regime within the $\mathrm{SO}(5)$-symmetric subspace of theory space. If the effective scaling dimensions of allowed $\mathrm{SO}(5)$ breaking perturbations-specifically, the symmetric tensor $X_{a b c d}^{(4)}$ discussed in Sec. IV D-are greater than three, the $\mathrm{NCCP}^{1}$ model and QED-Gross-Neveu model can lie in the basin of attraction of this regime, and will also show pseudocritical behavior. In this scenario the dualities we discuss apply to the physics at length scales up to $\xi$ (and somewhat beyond; see below).

To make the above possibility more concrete, we may think of $\mathrm{NCCP}^{1}$ as a perturbation of an exactly
$\mathrm{SO}(5)$-invariant theory whose RG behavior could in principle be pinned down. The nonlinear sigma model is one possibility: in the above scenario we would expect the sigma model at strong coupling to exhibit pseudocritical behavior. However, the language of the sigma model does not give us an obvious candidate for the deformation parameter $\tau$. Tentatively, a renormalizable alternative may be the $N_{c}=2, N_{f}=2 \mathrm{QCD}_{3}$ discussed in Sec. VI. This theory has an $\mathrm{SO}(5)$ symmetry which becomes explicit when it is written in terms of Majorana fermions. The theory may spontaneously break $\mathrm{SO}(5)$ on the scale set by the coupling, in which case it is not very interesting. But another possibility is that it generates a long length scale by the above mechanism. If so, $N_{f}$ and $d$ are candidates for deformation parameters $\tau$ which could produce a true fixed point.

Another way to think about the possibility of an $\mathrm{SO}(5)-$ invariant pseudocritical regime is to hypothesize an exact $\mathrm{SO}(5)$ symmetry for the nearby nonunitary fixed points at imaginary $\lambda$. We would then view the $\mathrm{NCCP}^{1}$ model and QED-Gross-Neveu model on length scales $\lesssim \xi$ as perturbations away from this fixed point.

As an aside, note that in the present scenario $\mathrm{SO}(5)$ symmetry survives to length scales even larger than $\xi$. The simplest possibility is that on scales larger than $\xi$ the system flows to the ordered phase of the $\mathrm{SO}(5)$ sigma model, representing a first-order transition for $\mathrm{NCCP}^{1}$ —although in principle it is possible that the theory could flow to a new nontrivial fixed point. This ordered sigma model is subject to anisotropies due to the $\mathrm{SO}(5)$-breaking perturbations that are allowed in the microscopic model. However, these anisotropies are small since the effective RG eigenvalue $y_{4}$ of the four-index symmetric tensor is negative in the pseudocritical regime: they do not become apparent until a length scale $L_{*} \sim \xi^{1+\left|y_{4}\right| / 3} \times$ (bare coupling). Simulations in the range $\xi \lesssim L \lesssim L_{*}$ would find four apparent Goldstone modes [161].

A similar conjecture could in principle apply to the dualities between $\mathrm{O}(4)$-invariant theories, although numerical results for the easy-plane Néel-VBS transition suggest that a first-order transition with a rather shorter correlation length than in the $\mathrm{SU}(2)$ case may be generic there [20,69-72]. As noted in Sec. III C, it is also conceivable that the fermionic theories flow to an $\mathrm{O}(4)$ symmetric fixed point (or pseudocritical point), but that the easy-plane model does not, as a result of an additional perturbation allowed by microscopic symmetry. Further numerical studies of both the easy-plane model and of $\mathrm{QED}_{3}$ along the lines described in Sec. VIII are clearly called for.

For quantum phase transitions, the pseudocriticality scenario implies that the system will show quantum critical behavior above a parametrically low-temperature scale $T^{*} \sim J \exp \left[-\pi / \sqrt{a\left(\tau-\tau_{c}\right)}\right]$, where $J$ is a microscopic energy scale, with critical exponents drifting as the 
temperature changes. Criticality eventually disappears below $T^{*}$, and the system possibly crosses over to a first-order transition. But for sufficiently low $T^{*}$, a quantum critical regime (the famous "critical fan") should be observable above $T^{*}$. Pseudocritical systems thus present interesting possibilities for phenomenology near quantum phase transitions.

\section{ACKNOWLEDGMENTS}

We thank F. Alet, J. Chalker, Y. C. He, R. Kaul, A. C. Potter, S. Powell, S. Pufu, S. Rychkov, A. Sandvik, N. Seiberg, P. Serna, D. Simmons-Duffin, A. Vishwanath, and E. Witten for helpful discussions. We thank D. SimmonsDuffin for sharing unpublished bootstrap calculations. C. W. is supported by the Harvard Society of Fellows. A. N. was supported by the Gordon and Betty Moore Foundation under the EPiQS initiative (Grant No. GBMF4303) and by the EPSRC Grant No. EP/ N028678/1. C.X. is funded by the David and Lucile Packard Foundation and NSF Grant No. DMR-1151208. T. S. is supported by a U.S. Department of Energy Grant No. DE-SC0008739, and in part by a Simons Investigator award from the Simons Foundation. C. W., M. A. M., and C.X. thank the hospitality of the Kavli Institute for Theoretical Physics, which is supported in part by the National Science Foundation under Grant No. NSF PHY1125915. Part of this work was performed by T. S. at the Aspen Center for Physics, which is supported by National Science Foundation Grant No. PHY-1066293. Research at Perimeter Institute for Theoretical Physics (M. A. M.) is supported by the Government of Canada through the Department of Innovation, Science and Economic Development and by the Province of Ontario through the Ministry of Research and Innovation.

\section{APPENDIX A: MORE PRECISE LAGRANGIANS}

In this Appendix, we present the easy-plane dualities in a more precise notation. We define a Dirac fermion through a Pauli-Villars regulator, such that its partition function [under a general U(1) gauge field $a$ and metric $g$ ] is given by $Z_{\psi}=\left|Z_{\psi}\right| e^{-i \pi \eta[a, g] / 2}$. In the usual notation (used in the main text), this would correspond to a Dirac Lagrangian supplemented with a Chern-Simons term at level $k=-1 / 2$ for both $a$ and $g$. In general, it is more precise to use the $\eta$ invariant instead of the less well-defined $k=-1 / 2$ ChernSimons terms (for more details, see, for example, Ref. [60] for a review).

We also enforce the proper quantization of all the $\mathrm{U}(1)$ gauge field (including dynamical and background ones) from the beginning, by working directly with $B_{1,2}\left(\right.$ instead of $\left.B, B^{\prime}\right)$ in the main text. The duality now reads:

$$
\begin{aligned}
&\left|D_{b} z_{1}\right|^{2}+\left|D_{b-B_{1}} z_{2}\right|^{2}-\left|z_{1}\right|^{4}-\left|z_{2}\right|^{4}+\frac{1}{2 \pi} b d B_{2} \\
& \Leftrightarrow \bar{\psi}_{1} i D_{a} \psi_{1}+\bar{\psi}_{2} i D_{a+B_{2}-B_{1}} \psi_{2}+\frac{1}{4 \pi} a d a+\frac{1}{2 \pi} a d B_{2} \\
&+\frac{1}{4 \pi} B_{2} d B_{2}+2 \mathrm{CS}_{g},
\end{aligned}
$$

where $\mathrm{CS}_{g}$ is a gravitational Chern-Simons term, normalized such that in the absence of any other matter field it leads to a thermal-Hall conductance $\kappa_{x y}=\frac{1}{2}\left[\left(\pi^{2} k_{B}^{2} T\right) / 3 h\right]$.

\section{APPENDIX B: SOME OTHER DUALITIES}

Here, we describe an alternate set of dualities between theories with global $\mathrm{SU}(2), \mathrm{U}(1)$, and $\mathcal{T}$ symmetries. We begin with a duality between $N=2$ species of a free massless Dirac fermion [162] and a bosonic theory.

To be concrete, we define the partition function of the massless Dirac fermion in terms of the $\eta$ invariant. To maintain SU(2) symmetry between the two species, we must choose the same regularization for both species. Therefore, we write the partition function of the $N=2$ free massless Dirac fermion as

$$
Z_{\psi}=\left|Z_{\psi}\right| e^{-i \pi \eta[A, g]} .
$$

Here, $A, g$ are the background gauge field (strictly speaking a $\operatorname{spin}_{c}$ connection) and metric, respectively. We know that $e^{-i \pi \eta[A, g]}=e^{-i \int(1 / 4 \pi) A d A+2 \mathrm{CS}[g]}$. Therefore, the theory can be made time-reversal invariant [while keeping $\mathrm{SU}(2)$ and $\mathrm{U}(1)]$ by adding $\int[1 /(4 \pi)] A d A+2 \mathrm{CS}[g]$. Thus, we consider [163]

$$
\mathcal{L}_{0 f}=i \bar{\psi}_{\alpha} D_{A} \psi_{\alpha}+\frac{1}{4 \pi} A d A+2 \mathrm{CS}[g] .
$$

We claim this has a dual bosonic description:

$$
\mathcal{L}_{0 b}=\mathcal{L}\left[Z_{\alpha}, b\right]+\frac{1}{2 \pi} b d A-\frac{1}{4 \pi} b d b,
$$

where $b$ is an ordinary $\mathrm{U}(1)$ gauge field and $Z_{\alpha}$ is a spin$1 / 2$ [under the global internal SU(2)] boson. As a check on this proposal, consider giving the fermions a mass that preserves $\mathrm{SU}(2) \times \mathrm{U}(1)$. If $m<0$ (with our definition of the fermion determinant), we get $\int[1 /(4 \pi)] A d A+2 \mathrm{CS}[g]$ (which corresponds to a gapped phase with $\sigma_{x y}=1$, $\left.\kappa_{x y}=1\right)$. If $m>0$, then we get the same but with opposite $\operatorname{sign}\left(\sigma_{x y}=-1, \kappa_{x y} / \kappa_{0}=-1\right)$. The massless Dirac fermion sits right at the transition between these two phases.

To match these from the boson side, if $Z_{\alpha}$ is in a trivial insulator, we integrate it out first, and then integrate out $b$. This gives $\int[1 /(4 \pi)] A d A+2 \mathrm{CS}[g]$, which exactly matches the fermion side with $m<0$. The other phase is obtained by putting $Z_{\alpha}$ in a boson integer quantum Hall state. Then 
integrating out $z_{\alpha}$ gives a term $[2 /(4 \pi)] b d b$. Combining with the other terms already in the boson action, and integrating out $b$, we get $-\left\{\int[1 /(4 \pi)] A d A+2 \mathrm{CS}[g]\right\}$, which also exactly matches the answer from the fermion side with $m>0$.

Therefore, what in the boson theory is the transition between a trivial insulator and the BIQH state is dual to the free massless Dirac fermion. This should not be surprising to us. In fact, we already know two different forms of actions that describe BIQH transitions: $\mathrm{QED}_{3}$ with $N_{f}=2$ and the easy-plane $\mathrm{NCCP}^{1}$. If we use the $\mathrm{QED}_{3}$ Lagrangian in Eq. (B3), we simply get back the free Dirac fermion theory. If we use the easy-plane $\mathrm{NCCP}^{1}$ theory instead, we obtain a "composite boson" dual of two free Dirac fermions discussed in Ref. [164]. However, in this representation the spin $\mathrm{SU}(2)$ symmetry is nonmanifest. Thus, in the absence of a manifestly SU(2)-invariant bosonic representation of the BIQH transition, we just keep the form of the Lagrangian in Eq. (B3) implicit.

We can also check monopole operators. A $2 \pi$ monopole $\mathcal{M}_{b}$ of $b$ carries $\mathrm{U}_{A}(1)$ charge 1 , and $\mathrm{U}_{b}(1)$ charge -1 . Thus, $Z_{\alpha} \mathcal{M}_{b}$ has no $\mathrm{U}_{b}(1)$ charge, has charge 1 under $\mathrm{U}_{A}(1)$, is an $\mathrm{SU}(2)$ doublet, and is a fermion. We should identify it with $\psi_{\alpha}$.

Now, we follow the usual logic to generate other dualities. First, we let $A \rightarrow a$ and treat $a$ as a dynamical gauge field $\left(\operatorname{spin}_{c}\right.$ connection), and couple $[1 /(2 \pi)] a d B+$ $[1 /(4 \pi)] B d B$, where $B$ is an ordinary $\mathrm{U}(1)$ gauge field. The fermion side becomes

$$
\mathcal{L}_{1 f}=i \bar{\psi}_{\alpha} D_{a} \psi_{\alpha}+\frac{1}{2 \pi} a d B+\frac{1}{4 \pi} B d B+2 \operatorname{CS}[g]
$$

In the boson side the $a$ dependence arises solely through the term $[1 /(2 \pi)](b+B) d a$. Integrating out $a$ we set $b=-B$ to get

$$
\mathcal{L}_{1 b}=\mathcal{L}\left[Z_{\alpha},-B\right]
$$

The two theories Eqs. (B4) and (B5) are dual to each other in the following sense. The phase transition in Eq. (B5) between the trivial gapped phase and the BIQH state is described by Eq. (B4) when the Dirac fermions are massless. Indeed, we used this identification in various parts of the paper.

Now we make $B$ dynamical $B \rightarrow b$, and couple $[1 /(2 \pi)] b d C$ where $C$ is an ordinary $\mathrm{U}(1)$ gauge field. In the fermion side the $b$ dependence occurs through the term $[1 /(2 \pi)](a+C) d b+[1 /(4 \pi)] b d b$. Integrating out $b$ leads to

$$
\mathcal{L}_{f}=i \bar{\psi}_{\alpha} D_{a} \psi_{\alpha}-\frac{1}{4 \pi} a d a+\frac{1}{2 \pi} a d C-\frac{1}{4 \pi} C d C .
$$

The boson side becomes

$$
\mathcal{L}_{b}=\mathcal{L}\left[Z_{\alpha},-b\right]+\frac{1}{2 \pi} C d b .
$$

This almost looks like the $\mathrm{NCCP}^{1}$ model, but we should remember the precise sense in which Eqs. (B6) and (B7) are dual. As in all the previous examples, the trivial to BIQH transition of $z_{\alpha}$ is dual to the massless Dirac theory. Let us interpret this phase transition in the boson side more clearly. When $z_{\alpha}$ is in a trivial gapped phase, $C$ is Higgsed, and we have broken $U_{C}(1)$ symmetry (a "superfluid"). When $z_{\alpha}$ is in a BIQH state, we have a $\mathrm{U}(1)_{2}$ theory, and this is really an $\mathrm{SU}(2)$-symmetric chiral spin liquid where the semion is a spin- $1 / 2$ spinon.

Thus, the transition between a superfluid [that breaks $\mathrm{U}(1)$ but preserves $\mathrm{SU}(2)$ ] and this chiral spin liquid with $\mathrm{SU}$ (2) symmetry is described by Eq. (B6). It is easy to check that this is reproduced by thinking directly about the fermions.

The duality between the theories in Eq. (B7) (interpreted as above) and Eq. (B6) should be contrasted with the duality of the $\mathrm{SU}(2)$-invariant $\mathrm{NCCP}^{1}$ model to the $\mathrm{QED}_{3}$ - GN model. The two sets of dualities describe two distinct phase transitions of the same underlying spin system. Further, though Eq. (B7) has the same $\mathrm{SO}(3) \times \mathrm{U}(1)$ symmetry as $\mathrm{NCCP}^{1}$, it—unlike $\mathrm{NCCP}^{1}$ is not time-reversal invariant.

\section{APPENDIX C: A DIFFERENT VIEW OF THE DUALITIES AND EMERGENT SYMMETRIES}

Here, we discuss the dualities and emergent symmetries from a point of view familiar in the high-energy literature. However, caution is needed, as we describe below.

For any $(2+1)$ D CFT with a global U(1) symmetry, there is a formal operation on the path integral, denoted $S$, which is defined as follows:

$$
Z_{S}[B]=\int \mathcal{D} A Z_{\mathrm{CFT}_{1}}[A] e^{(i / 2 \pi) \int d^{3} x A d B} .
$$

Here, $Z_{\mathrm{CFT}_{1}}[A]$ is the partition function of the $(2+1) \mathrm{D}$ CFT in the presence of a background $\mathrm{U}(1)$ gauge field $A$. The operation $S$ converts this background gauge field into a dynamical one, without including a kinetic term for the field $A$. A new background $\mathrm{U}(1)$ gauge field $B$, coupling to $d A / 2 \pi$ (which is conserved), is also introduced. This operation was defined and used by Kapustin and Strassler [49], and by Witten [50]. A different operation, $T$, was also introduced by Witten: this simply shifts the level of the Chern-Simons term for the background gauge field by 1 .

If the path integral on the right-hand side of Eq. (C1) is well defined, then $Z_{S}[B]$ is the partition function of a new theory with a new global $U(1)$ symmetry ( $B$ couples to the current of this symmetry). Further, the theory $Z_{S}[B]$ is conformally invariant, at least at the formal level-it is to ensure that no kinetic term for $A$ is introduced in the 
definition of $S$ - and defines a new conformal field theory which we denote $\mathrm{CFT}_{2}$.

Schematically we write the $S$ operation as $S\left[\mathrm{CFT}_{1}\right]=$ $\mathrm{CFT}_{2}$, where both CFTs have a global U(1) symmetry. The combination of $S$ and $T$ then leads to a remarkable $S L(2, Z)$ action on the set of $(2+1) \mathrm{D}$ CFTs with a global U(1) symmetry [50]. (See Refs. [165-168] for other appearances of this mathematical structure in related contexts.) That is, $S$ and $T$ can be shown formally to satisfy the defining relations [169] $S^{2}=-1$ and $(S T)^{3}=1$ of $S L(2, Z)$.

Let us think a bit more about $S$. A priori it is not evident that the path integral in Eq. (C1) is well defined. To obtain some intuition, consider a modified operation that is certainly well defined:

$$
\begin{aligned}
& \tilde{Z}_{S}\left[B ; e^{2}\right] \\
& \quad=\int \mathcal{D} A Z_{\mathrm{CFT}_{1}}[A] \exp \left[-\int d^{3} x\left(\frac{1}{2 e^{2}}(d A)^{2}-\frac{i}{2 \pi} A d B\right)\right] .
\end{aligned}
$$

We introduce a Maxwell term for the gauge field $A$ with coupling constant $e^{2}$. Formally, the original $S$ operation may be written as

$$
Z_{S}[B]=\lim _{e^{2} \rightarrow \infty} \tilde{Z}_{S}\left[B ; e^{2}\right] .
$$

However, Eq. (C3) has a more intuitive interpretation. Consider the theory at a fixed value of $e^{2}$. The gauge coupling introduces a length scale $l_{e} \sim 1 / e^{2}$. At distances much smaller than $l_{e}$ the physics is that of $\mathrm{CFT}_{1}$ plus a decoupled free photon, so this is a "weakly" gauged version of $\mathrm{CFT}_{1}$. But the coupling between $\mathrm{CFT}_{1}$ and the photon is relevant, so the physics on distances much larger than $l_{e}$ will be different. The limit $e^{2} \rightarrow \infty$ is equivalent to describing the deep IR limit of the $\tilde{Z}_{S}$ theory, i.e., distances much greater than $l_{e}$.

At the formal level this deep IR limit is a new conformally invariant theory, $\mathrm{CFT}_{2}$, described by $Z_{S}$. Formally, the relationship $S^{2}=-1$ (see below) also requires $\mathrm{CFT}_{2}$ to be nontrivial if $\mathrm{CFT}_{1}$ is. But it is not obvious that the conclusions of these formal arguments will always hold in reality, at least for the nonsupersymmetric, finite " $N$ " theories of interest in this paper. For instance, if we take $\mathrm{CFT}_{1}$ to be the theory of $N_{f}$ massless twocomponent Dirac fermions ( $N_{f}$ even), then we obtain $\mathrm{QED}_{3}$ for $\tilde{Z}_{S}$. Whether or not this flows to a CFT for general $N_{f}$, not necessarily large, is a long-standing issue that has not yet been settled. For another example relevant to this paper, take $\mathrm{CFT}_{1}$ to describe a pair of boson fields, each separately at the U(1) Wilson-Fisher fixed point, and take $S$ to act on the diagonal U(1) symmetry. $\tilde{Z}_{S}$ then describes the easy-plane $\mathrm{NCCP}^{1}$ model in a particular limit [170]. As discussed in the main text, it is hardly clear that this flows to a CFT in the IR. Similarly, if we start with the $\mathrm{O}(4)$ Wilson-Fisher theory and use $S$ to gauge an appropriate $\mathrm{U}(1)$ subgroup, we obtain the $\mathrm{SU}(2)$-symmetric $\mathrm{NCCP}^{1}$ model (in a similar limit). Whether or not this flows to a CFT is again a nontrivial question [171]. (Even when there is a flow to a CFT, we might expect to have to tune the coupling of any relevant symmetry-allowed operators of $\mathrm{CFT}_{1}$ in order to be on this flow line, contrary to the expectation from the formal limit.)

What about the crucial relationship $S^{2}=-1$ ? We apply the limiting procedure twice to give the partition function

$$
Z_{S^{2}}[C]=\lim _{e^{\prime 2} \rightarrow \infty} \lim _{e^{2} \rightarrow \infty} \tilde{Z}_{S^{2}}\left[C ; e^{2}, e^{\prime 2}\right],
$$

with

$$
\begin{aligned}
\tilde{Z}_{S^{2}}\left[C ; e^{2}, e^{\prime 2}\right] \\
=\int \mathcal{D} B \int \mathcal{D} A Z_{\mathrm{CFT}_{1}}[A] \\
\quad \times \exp \left[-\int d^{3} x\left(\frac{(d A)^{2}}{2 e^{2}}+\frac{(d B)^{2}}{2 e^{\prime 2}}-\frac{i}{2 \pi} B d(C+A)\right)\right] .
\end{aligned}
$$

The proof in Ref. [50] of $S^{2}=-1$ evaluates the path integral above in the absence of the Maxwell terms [as appropriate to the formal definition of $S$ in Eq. (C1)]. The $B$ integral then acts as a delta function enforcing $C=-A$, and the right-hand side becomes $Z_{\mathrm{CFT}_{1}}[-C]$.

Even in the case where $l_{e} \gg l_{e^{\prime}}$,

$$
\lim _{e^{2} \rightarrow \infty} \lim _{e^{\prime 2} \rightarrow \infty} \tilde{Z}_{S^{2}}\left[C ; e^{2}, e^{\prime 2}\right]
$$

we may worry that this procedure will fail to give back the original CFT, due to relevant terms generated by integrating out $B$ and then $A$. For Eq. (C4), where the order of limits is the opposite, it is even less clear that we will obtain $S^{2}=-1$. In general, this is a nontrivial question about the structure of the RG flows.

If the gauged $\mathrm{CFT}_{1}$ does indeed flow to a nontrivial $\mathrm{CFT}_{2}$, then the characteristic length scale for this crossover is $l_{e} \sim 1 / e^{2}$. Gauging $\mathrm{CFT}_{2}$ then introduces a new length scale $l_{e^{\prime}} \sim 1 / e^{\prime 2}$, and the regime of interest is $l_{e^{\prime}} \gg l_{e}$. In order for $S^{2}=-1$ to hold, the ultimate flow on scales $\gg l_{e^{\prime}}$ must be to a copy of the $\mathrm{CFT}_{1}$ fixed point.

If the gauged $\mathrm{CFT}_{1}$ instead flows to a trivial theory, representing, for example, a massive or symmetry-broken fixed point, then it is hard to see how $S^{2}=-1$ can ever be satisfied. Here, we are assuming that $\mathrm{CFT}_{1}$ is nontrivial; it is certainly possible to have an example where $\mathrm{CFT}_{1}$ and $\mathrm{CFT}_{2}$ are both trivial, and $S^{2}=-1$ [172].

This discussion is intended to provide intuition for dangers that may arise in the formal use of the $S$ operation. 
It is of course conceivable that in practice they do not arise. We should also emphasize that the limiting procedure discussed above is not the only way to interpret the formal definition of $Z_{S}$ : it is possible that when the above limits fail to give a nontrivial CFT, the $S$ operation can be rescued by an alternative implementation of the definition.

If we ignore all the caveats and assume (as is normally done in the literature) that there is a well-defined $S L(2, Z)$ action on $(2+1)$ D CFTs, then we can make some powerful statements. First, it tells us that there is a CFT that looks like an easy-plane $\mathrm{NCCP}^{1}$, defined by the partition function

$$
\int \mathcal{D} b\left(Z_{\mathrm{WF}}[b]\right)^{2} e^{i \int(1 / 2 \pi) b d B},
$$

where $Z_{\mathrm{WF}}[b]$ is the partition function for the Wilson-Fisher fixed point of a single complex boson, with background gauge field $b$. A priori we do not know whether the standard easy-plane $\mathrm{NCCP}^{1}$ action (defined with, e.g., an additional Maxwell term) flows to this CFT.

Second, if we assume that the formal $S$ operation gives a well-defined action on CFTs, then it is natural to expect that basic boson-fermion duality, relating a single massless Dirac fermion to a Wilson-Fisher boson coupled to a $\mathrm{U}(1)_{1}$ gauge field, can be taken as an exact statement about path integrals:

$$
Z_{D}[A]=\int \mathcal{D} b Z_{\mathrm{WF}}[b] e^{i \int d^{3} x(1 / 4 \pi) b d b+(1 / 2 \pi) b d A},
$$

where $Z_{D}$ is the partition function of a free massless Dirac fermion. We write this as (recall that $T$ shifts the level of the Chern-Simons term)

$$
D=S T[\mathrm{WF}] .
$$

The other boson-fermion duality then is

$$
D=T^{-1} S^{-1} T^{-1}[\mathrm{WF}]
$$

Multiplying the partition functions on both sides, shifting an $A d A / 4 \pi$ to the left, and finally making $A$ dynamical, we get the duality of $\mathrm{QED}_{3}$ to the easy-plane CFT defined in Eq. (C7).

The fermion side is manifestly $[\mathrm{SU}(2) \times \mathrm{U}(1)] / Z_{2}$ invariant. Further, it is easy to see that it is exactly selfdual and the dual side has the other $[\mathrm{SU}(2) \times \mathrm{U}(1)] / Z_{2}$ as a manifest symmetry. Altogether this implies the $\mathrm{O}(4)$ symmetry.

Within the framework of the present assumptions, these are all exact statements, regardless of the relevance or irrelevance of operators that break $\mathrm{O}(4)$ to $\mathrm{SU}(2) \times \mathrm{U}(1)$ or $\mathrm{U}(1) \times \mathrm{U}(1)$. As mentioned above, it is possible that the fixed point we are describing is highly fine-tuned. But if we now make the natural further assumption that the weak coupling limits of all these gauge theories flow to the IR CFTs defined formally but exactly by the path integrals above, and that there is no fine-tuning hidden in this flow, we indeed conclude that the various symmetry-allowed perturbations are irrelevant. However, we emphasize again that this view on the dualities and emergent symmetries is predicated on the reliability of the formal $S L(2, Z)$ action on $(2+1) \mathrm{D}$ CFTs, which as far as we are aware still remains conjectural.

\section{APPENDIX D: SOME USEFUL MATHEMATICAL CONCEPTS}

It is convenient to consider a "triangulation" of the spacetime manifold $M$ (we are mostly interested in manifolds of dimension $D=4$ ): we represent points in spacetime by a discrete lattice where each elementary unit is a $D$ simplex. Pick a local ordering of the vertices of the lattice. A $k$-cochain lives on $k$-simplices, i.e., it is a function that depends on $(k+1)$ vertices and takes values in some Abelian group $G$. We only need to consider the cases $Z, Z_{2}$, and $Z_{4}$. The corresponding cochain is then said to be an element of $C^{k}(M, G)$. For instance, a 2-cochain in $C^{2}\left(M, Z_{2}\right)$ is a function $a_{i j k}=0,1$, while for a 2-cochain in $C^{2}(M, Z)$, the function $a_{i j k} \in Z$. Here, $(i j k)$ are the vertices of a triangular plaquette of the simplex.

We can define a discrete derivative (known as a "coboundary") operation $d$ that maps $k$-cochains to $(k+1)$-cochains:

$$
(d a)_{\left(i_{0}, i_{1}, i_{2}, \ldots, i_{k+1}\right)}=\sum_{p=0}^{k+1}(-1)^{p} a_{i_{0}, i_{1}, \ldots, \hat{i}_{p}, \ldots, i_{k+1}},
$$

where the variable $\hat{i_{p}}$ is omitted. It is understood that the addition on the right-hand side is performed in $G$ (e.g., mod 2 addition for $G=Z_{2}$ ). It is readily checked that $d^{2} a=0$. The set of all $k$-cochains $a$ that satisfy $d a=0$ form a group under addition known as the cocycle group $Z^{k}(M, G)$. The set of all $a \in C^{k}(M, G)$ that may be written $a=d b$ for some $b \in C^{(k-1)}(M, G)$ form a different group known as the coboundary group $B^{k}(M, G)$. Clearly, $B^{k}(M, G) \subset Z^{k}(M, G)$. The cohomology group $H^{k}(M, G)=$ $\left\{\left[Z^{k}(M, G)\right] / B^{k}(M, G)\right\}$.

For two 2-cochains $a \in C^{k}(M, G)$ and $b \in C^{l}(M, G)$, we define the cup product

$$
(a \cup b)_{i_{0}, \ldots, i_{k+l}}=a_{\left.i_{0} i_{1} i_{2} \ldots i_{k}\right)} b_{i_{k} i_{k+1} \ldots i_{k+l}},
$$

where $i_{0}, \ldots, i_{k+l}$ are assumed to be ordered. The cup product satisfies

$$
d(a \cup b)=d a \cup b+(-1)^{k} a \cup d b .
$$

Clearly, if $d a=0=d b$, then $d(a \cup b)=0$. Thus, the cup product defines a product of cohomology classes. The cup 
product is a generalization of the familiar wedge product of differential forms.

The Poyntryagin square of $w \in H^{2}\left(M, Z_{2}\right)$ plays an important role in our discussion (see Ref. [173] for more discussion and references). We now specialize to fourmanifolds $M=Y_{4}$. It is easiest to define if $w$ can be lifted to an element $\hat{w} \in H^{2}\left(Y_{4}, Z\right)$; i.e., $w=\hat{w}(\bmod 2)$ and $d \hat{w}=0$. In this case, $\mathcal{P}(w)=\hat{w} \cup \hat{w} \bmod 4$. If $w$ does not admit a lift to an integral cohomology class, then $\mathcal{P}$ is still a mod 4 quantity. It is defined to be

$$
\mathcal{P}(w)=w \cup w+w \cup_{1} d w \quad(\bmod 4) .
$$

The new product $\cup_{1}$ is defined (for a 2-cochain $a$ and a 3-cochain $b$ ) as

$$
\left(a \cup_{1} b\right)_{01234}=a_{034} b_{0123}+a_{014} b_{1234} .
$$

It is readily seen that $\mathcal{P}(w)$ transforms by a coboundary under $w \rightarrow w+2 n, w \rightarrow w+d m$, so it is well defined on $H^{2}\left(Y_{4}, Z_{2}\right)$. Note that as $w \in H^{2}\left(Y_{4}, Z_{2}\right), d w=0 \bmod 2$. Thus, we have $\mathcal{P}(w)=w \cup w \bmod 2$. It can be shown that

$$
\mathcal{P}\left(w+w^{\prime}\right)=\mathcal{P}(w)+\mathcal{P}\left(w^{\prime}\right)+2 w \cup w^{\prime} \quad(\bmod 4) .
$$

We use this repeatedly.

\section{APPENDIX E: TOPOLOGICAL SUPERCONDUCTORS AND THE APS THEOREM}

In this Appendix, we review the field-theoretic description of topological superconductors in $(2+1) \mathrm{D}$ and $(3+1)$ D. We follow Ref. [60] here and adapt it to the $\mathrm{SO}(n)$-symmetric systems of interest in this paper.

Let us begin with $(2+1) \mathrm{D}$. A $p_{x}+i p_{y}$ superconductor can be represented by a massive two-component Majorana fermion $\chi$ :

$$
L=\bar{\chi}(D+m) \chi,
$$

with $\bar{\chi}=\chi^{T} C^{*}, D=\gamma^{\mu}\left(\partial_{\mu}+i \omega_{\mu}\right)$, and $C=\sigma^{y}$ the chargeconjugation matrix. $m>0$ corresponds to the trivial superconductor and $m<0$ to the $p_{x}+i p_{y}$ superconductor. The point $m=0$ corresponds to the transition between these two phases. Now, the formal partition function of Eq. (E1) on a closed manifold $M$ is

$$
\begin{aligned}
Z(m)=\operatorname{Pf}\left(C^{\dagger}(D+m)\right) & = \pm \operatorname{det}(D+m)^{1 / 2} \\
& = \pm \prod_{\langle\lambda\rangle}(-i \lambda+m)^{N(\lambda) / 2},
\end{aligned}
$$

where the product is over eigenvalues $\lambda$ of the Dirac operator $i D$ (without repetitions) and $N(\lambda)$ is the multiplicity of the eigenvalue. Since $[C K, i D]=0$ and
$(C K)^{2}=-1$, all eigenvalues of $i D$ are doubly degenerate. The above expression clearly requires regularization. We note that the partition function of the trivial superconductor at long wavelength (or equivalently in the $m \rightarrow \infty$ limit) is expected to be analytic in the curvature of the manifold and to have no topological terms, so it can be effectively set to 1 . It is then convenient to normalize other partition functions by it. This can be understood as the physical justification of Pauli-Villars regularization. Then,

$$
Z(m)_{\mathrm{PV},+}=\lim _{M \rightarrow \infty} \frac{Z_{m}}{Z_{M}}=\prod_{\langle\lambda\rangle} \frac{(-i \lambda+m)^{N(\lambda) / 2}}{(-i \lambda+|M|)^{N(\lambda) / 2}} .
$$

Note that there is no sign ambiguity in Eq. (E3). Indeed, we can reach any value of $m$ starting with the trivial insulator at $m=\infty$. The requirement that the partition function during this process be analytic in $m$ removes the sign ambiguity. Now, when $m=0$, we can write the partition function Eq. (E3) as $Z=|Z| e^{i \varphi}$, where the phase

$\varphi=-\frac{1}{2} \sum_{\langle\lambda \neq 0\rangle} N(\lambda) \operatorname{sgn}(\lambda) \tan ^{-1} \frac{|M|}{|\lambda|} \rightarrow-\frac{\pi}{4} \sum_{\lambda \neq 0} \operatorname{sgn}(\lambda)$.

The sum in the last term is over all eigenvalues of $i D$ (repeated eigenvalues included), and we take the $M \rightarrow \infty$ limit naively. While the resulting final sum is formal, it can be equivalently regulated with the $\zeta$ function method, giving

$$
Z(m=0)_{\mathrm{PV},+}=|Z(m=0)| \exp [-\pi i \eta(i D) / 4],
$$

with $\eta$ defined via Eq. (116) [174]. Deep in the $p_{x}+i p_{y}$ phase, we may set $m=-|M| \rightarrow-\infty$ in Eq. (E3) and obtain a pure phase,

$$
Z_{p_{x}+i p_{y}}=\exp [-\pi i \eta(i D) / 2] .
$$

The APS theorem Eq. (120) allows us to rewrite the partition function of a $p_{x}+i p_{y}$ superconductor, Eq. (E6), as

$$
Z_{p_{x}+i p_{y}}=\exp \left(-i \mathrm{CS}_{g}\left[Y_{4}\right]\right)
$$

where, as we explain in Sec. VII, the gravitational Chern-Simons term CS $_{g}$ [Eq. (119)] is defined via a continuation of $M$ to an auxiliary four-manifold $Y_{4}$. The APS theorem guarantees that the result is independent of the continuation. The gravitational Chern-Simons term encodes precisely the thermal-Hall response of a $p_{x}+i p_{y}$ superconductor: $\left[\left(\kappa_{x y}\right) / T\right]=\frac{1}{2}$. Note that our Majorana fermions $\chi$ require a spin structure, and the continuation of $M$ to $Y_{4}$ must preserve this spin structure. Thus, $\mathrm{CS}_{g}\left[Y_{4}\right]$ is secretly spin-structure dependent (as, less surprisingly, is the $\eta$ invariant). 
We can easily generalize the above discussion to $n$ identical Majorana fermions. Now we may couple the system to an $\mathrm{SO}(n)$ gauge field $A$, so that the Dirac operator reads $D_{A}=\gamma^{\mu}\left(\partial_{\mu}+i \omega_{\mu}-i A_{\mu}\right)$ [175]. The partition function for $m \rightarrow-\infty$ (i.e., deep in the topological phase) again is Eq. (E6) with $\eta(i D)$ now referring to the full Dirac operator $D_{A}$. This can be rewritten using the APS theorem Eq. (120) as

$$
Z_{\left(p_{x}+i p_{y}\right)^{n}}=\exp \left\{-i\left(\mathrm{CS}_{\mathrm{SO}(n)}\left[A, Y_{4}\right]+n \mathrm{CS}_{g}\left[Y_{4}\right]\right)\right\},
$$

with the $\mathrm{SO}(n)$ Chern-Simons term, given by Eq. (119), again defined via the continuation to $Y_{4}$. Thus, $n$ copies of a $p_{x}+i p_{y}$ superconductor have $\mathrm{SO}(n)$ response characterized by a Chern-Simons term at level 1 . For $n=2$, this simply corresponds to $\sigma_{x y}=1$. There is also the expected thermal-Hall response $\left[\left(\kappa_{x y}\right) / T\right]=n \times \frac{1}{2}$ encoded in the gravitational Chern-Simons term. As we emphasize in Sec. VII, for even $n$ the Majorana fermions really see an $\left[\operatorname{SO}(n) \times \operatorname{Spin}(3)_{T M}\right] / Z_{2}$ bundle; i.e., we do not need to separately specify the spin structure, but only the combination of $\mathrm{SO}(n)$ and $\operatorname{Spin}(3)_{T M}$ transition functions. Likewise, on $Y_{4}$ we again need to continue just the $\left[\mathrm{SO}(n) \times \operatorname{Spin}(3)_{T M}\right] / Z_{2}$ bundle. Finally, the partition function for $m=0$ (the transition point between a trivial phase and $n$ copies of a $p_{x}+i p_{y}$ superconductor) is again given by Eq. (E5).

Now, let us proceed to $(3+1) D$. A topological superconductor in class DIII can be represented by a massive (four-component) Majorana fermion. For generality, we work from the start with $n$ identical copies of a topological superconductor and couple the system to an $\mathrm{SO}(n)$ gauge field $A$, so the continuum bulk action is

$$
L=\bar{\chi}\left(D_{A}+m\right) \chi,
$$

where again $D_{A}=\gamma^{\mu}\left(\partial_{\mu}+i \omega-i A_{\mu}\right), \bar{\chi}=\chi^{T} C^{*}$, and $C$ is the charge-conjugation matrix. The phase with $m>0$ may be taken (by convention) to represent the trivial superconductor and $m<0$ is the topological superconductor. As before, we may set the partition function of the trivial phase, $m \rightarrow \infty$, to 1 , so the bulk partition function of the topological phase, $m \rightarrow-\infty$, on a closed four-manifold $X_{4}$ is

$$
Z_{\mathrm{TSc}}=\prod_{\langle\lambda\rangle} \frac{(-i \lambda-|M|)^{N(\lambda) / 2}}{(-i \lambda+|M|)^{N(\lambda) / 2}},
$$

with $\lambda$ eigenvalues of $i D_{A}$. Crucially, again $\left[C K, i D_{A}\right]=0$ and $(C K)^{2}=-1$, so all eigenvalues are doubly degenerate. What is different compared to the previously discussed $p_{x}+i p_{y}$ case is that $\left\{\gamma^{5}, i D_{A}\right\}=0$, so all nonzero eigenvalues of $i D_{A}$ come in pairs $\pm \lambda$; therefore, their contribution to the partition function cancels and

$$
Z_{\mathrm{TSc}}=(-1)^{N_{0} / 2},
$$

where $N_{0}$ is the number of zero modes of $i D_{A}$. The zero modes can be chosen to be simultaneous eigenstates of $\gamma^{5}$. Suppose there are $N_{ \pm}$eigenstates with $\gamma^{5}= \pm 1$. We note that $N_{+}$and $N_{-}$are separately even as $\left[C K, \gamma^{5}\right]=0$. Therefore, we may rewrite $Z_{\mathrm{TSc}}=(-1)^{\left(N_{+}-N_{-}\right) / 2}$. The difference $N_{+}-N_{-}=2 \mathcal{J}$ is known as the index of $i D_{A}$ (we include a prefactor of 2 to emphasize that in the present situation it is even), and we may write

$$
Z_{\mathrm{TSc}}=(-1)^{\mathcal{J}} \text {. }
$$

We see that the partition function is real, as it should be for a time-reversal invariant system on an orientable manifold. The Atiyah-Singer theorem [111,114] tells us that

$$
\begin{aligned}
2 \mathcal{J}=N_{+}-N_{-} & =\frac{1}{\pi}\left(\mathrm{CS}_{\mathrm{SO}(n)}\left[A, X_{4}\right]+n \mathrm{CS}_{g}\left[X_{4}\right]\right) \\
& =p_{1}\left[A, X_{4}\right]-\frac{n \sigma\left[X_{4}\right]}{8},
\end{aligned}
$$

with the Pontryagin number $p_{1}$ and signature $\sigma$ given by Eqs. (124) and (125), so that we may rewrite

$$
Z_{\mathrm{TSc}}=\exp \left[\frac{i}{2}\left(\mathrm{CS}_{\mathrm{SO}(n)}\left[A, X_{4}\right]+n \mathrm{CS}_{g}\left[X_{4}\right]\right)\right] .
$$

When $n=1$ (or more generally for odd $n$ ), we must pick a spin structure for our fermions $\chi$ (in particular, $X_{4}$ must admit a spin structure); we then learn from Eq. (E13) that on a spin manifold $\sigma$ is a multiple of 16. Furthermore, if we fix a spin structure, $A$ is a true $\mathrm{SO}(n)$ gauge field (with transition functions satisfying the cocycle condition), from which we learn that $p_{1}[A]$ on a spin manifold is even. Now, for even $n$, we do not require $X_{4}$ to admit a spin structure: the fermions see transition functions in the $\left[\mathrm{SO}(n) \times \operatorname{Spin}(4)_{T X_{4}}\right] / Z_{2}$ group, so while $p_{1}$ and $[(n \sigma) / 8]$ themselves need not be even (in fact, $\sigma$ is an integer, so $[(n \sigma) / 8]$ is generally a fraction), the combination $p_{1}-[(n \sigma) / 8]$ is an even integer.

Finally, let us discuss the case when the topological superconductor lives on a space $X_{4}$ with a boundary $M$. We know that the boundary supports $n$ gapless Majorana cones. The bulk + boundary partition function now is [60]

$$
\begin{aligned}
S_{\mathrm{TSc}}^{\text {bulk+bound }}= & \int_{M}\left[\bar{\chi} D_{A} \chi\right]_{\mathrm{PV},+} \\
& -\frac{i}{2}\left(\mathrm{CS}_{\mathrm{SO}(n)}\left[A, X_{4}\right]+n \mathrm{CS}_{g}\left[X_{4}\right]\right) .
\end{aligned}
$$

The Majorana action Eq. (E15) depends only on the boundary data; on the other hand, the second term in Eq. (E15) depends on the bulk, and, in fact, reduces to our previous expression Eq. (E14) for a closed manifold. While 
each term in Eq. (E15) is separately well defined, the timereversal symmetry of Eq. (E15) is not obvious. However, using Eq. (E5) and the APS theorem Eq. (120), we obtain

$$
Z_{\mathrm{TSc}}^{\text {bulk+bound }}=\left|Z^{\text {bound }}(m=0)\right|(-1)^{\mathcal{J}\left[A, X_{4}\right]},
$$

where $\left|Z^{\text {bound }}(m=0)\right|$ is the absolute value of the boundary Majorana fermion partition function, and $2 \mathcal{J}$ is the index of the bulk Dirac operator $i D_{A}$ with APS boundary conditions. The time-reversal symmetry is now manifest.

To obtain further physical intuition for the action Eq. (E15), we may break time-reversal symmetry on the surface with a mass term $m \bar{\chi} \chi$. Integrating the Majorana fermions out, by our preceding discussion we then obtain at long wavelength

$$
S=\mp \frac{i \operatorname{sgn}(m)}{2}\left(\mathrm{CS}_{\mathrm{SO}(n)}\left[A, X_{4}\right]+n \mathrm{CS}_{g}\left[X_{4}\right]\right) ;
$$

i.e., the surface has $\mathrm{SO}(n)$ Chern-Simons response at level $1 / 2$ and thermal-Hall response with $\kappa_{x y} / T=n \times \frac{1}{4}$. This is precisely what we expect for the $T$-broken surface state of $n$ copies of a topological superconductor.

[1] M.E. Peskin, Mandelstam-'t Hooft Duality in Abelian Lattice Models, Ann. Phys. (N.Y.) 113, 122 (1978).

[2] C. Dasgupta and B. I. Halperin, Phase Transition in a Lattice model of Superconductivity, Phys. Rev. Lett. 47, 1556 (1981).

[3] M. P. A. Fisher and D. H. Lee, Correspondence between Two-Dimensional Bosons and a Bulk Superconductor in a Magnetic Field, Phys. Rev. B 39, 2756 (1989).

[4] T. Senthil, A. Vishwanath, L. Balents, S. Sachdev, and M. P. A. Fisher, Deconfined Quantum Critical Points, Science 303, 1490 (2004).

[5] T. Senthil, L. Balents, S. Sachdev, A. Vishwanath, and M. P. A. Fisher, Quantum Criticality beyond the LandauGinzburg-Wilson Paradigm, Phys. Rev. B 70, 144407 (2004).

[6] A.W. Sandvik, Evidence for Deconfined Quantum Criticality in a Two-Dimensional Heisenberg Model with Four-Spin Interactions, Phys. Rev. Lett. 98, 227202 (2007).

[7] R. G. Melko and R. K. Kaul, Scaling in the Fan of an Unconventional Quantum Critical Point, Phys. Rev. Lett. 100, 017203 (2008).

[8] J. Lou, A. W. Sandvik, and N. Kawashima, Antiferromagnetic to Valence-Bond-Solid Transitions in Two-Dimensional $\mathrm{SU}(n)$ Heisenberg Models with Multispin Interactions, Phys. Rev. B 80, 180414 (2009).

[9] A. Banerjee, K. Damle, and F. Alet, Impurity Spin Texture at a Deconfined Quantum Critical Point, Phys. Rev. B 82, 155139 (2010).

[10] A. W. Sandvik, Continuous Quantum Phase Transition between an Antiferromagnet and a Valence-Bond Solid in
Two Dimensions: Evidence for Logarithmic Corrections to Scaling, Phys. Rev. Lett. 104, 177201 (2010).

[11] K. Harada, T. Suzuki, T. Okubo, H. Matsuo, J. Lou, H. Watanabe, S. Todo, and N. Kawashima, Possibility of Deconfined Criticality in $\mathrm{SU}(n)$ Heisenberg Models at Small n, Phys. Rev. B 88, 220408 (2013).

[12] F.-J. Jiang, M. Nyfeler, S. Chandrasekharan, and U.-J. Wiese, From an Antiferromagnet to a Valence Bond Solid: Evidence for a First-Order Phase Transition, J. Stat. Mech. (2008) P02009.

[13] K. Chen, Y. Huang, Y. Deng, A. B. Kuklov, N. V. Prokof'ev, and B. V. Svistunov, Deconfined Criticality Flow in the Heisenberg Model with Ring-Exchange Interactions, Phys. Rev. Lett. 110, 185701 (2013).

[14] A. Nahum, J. T. Chalker, P. Serna, M. Ortuño, and A. M. Somoza, Deconfined Quantum Criticality, Scaling Violations, and Classical Loop Models, Phys. Rev. X 5, 041048 (2015).

[15] A. Nahum, P. Serna, J. T. Chalker, M. Ortuño, and A. M. Somoza, Emergent SO(5) Symmetry at the Néel to Valence-Bond-Solid Transition, Phys. Rev. Lett. 115, 267203 (2015).

[16] O. I. Motrunich and A. Vishwanath, Comparative Study of Higgs Transition in One-Component and Two-Component Lattice Superconductor Models, arXiv:0805.1494.

[17] A. B. Kuklov, M. Matsumoto, N. V. Prokof'ev, B. V. Svistunov, and M. Troyer, Deconfined Criticality: Generic First-Order Transition in the SU(2) Symmetry Case, Phys. Rev. Lett. 101, 050405 (2008).

[18] L. Bartosch, Corrections to Scaling in the Critical Theory of Deconfined Criticality, Phys. Rev. B 88, 195140 (2013).

[19] D. Charrier, F. Alet, and P. Pujol, Gauge Theory Picture of an Ordering Transition in a Dimer Model, Phys. Rev. Lett. 101, 167205 (2008).

[20] G. Chen, J. Gukelberger, S. Trebst, F. Alet, and L. Balents, Coulomb Gas Transitions in Three-Dimensional Classical Dimer Models, Phys. Rev. B 80, 045112 (2009).

[21] D. Charrier and F. Alet, Phase Diagram of an Extended Classical Dimer Model, Phys. Rev. B 82, 014429 (2010).

[22] G. J. Sreejith and S. Powell, Scaling Dimensions of Higher-Charge Monopoles at Deconfined Critical Points, Phys. Rev. B 92, 184413 (2015).

[23] It is not yet clear whether the transition is truly second order or whether it displays only "quasiuniversal" behavior up to a very large but finite length scale [10-14,17,24-26]. We discuss this in detail in the text.

[24] H. Shao, W. Guo, and A. W. Sandvik, Quantum Criticality with Two Length Scales, Science 352, 213 (2016).

[25] D. Simmons-Duffin (unpublished).

[26] Y. Nakayama and T. Ohtsuki, Necessary Condition for Emergent Symmetry from the Conformal Bootstrap, Phys. Rev. Lett. 117, 131601 (2016).

[27] The continuous global symmetry of the theory is actually $\mathrm{SO}(3) \times \mathrm{O}(2)$ and not $\mathrm{SU}(2) \times \mathrm{U}(1)$ (see Sec. IV C). By a slight but standard abuse of terminology, we nevertheless refer to it as the $\mathrm{SU}(2) \mathrm{NCCP}^{1}$ model.

[28] A. Tanaka and X. Hu, Many-Body Spin Berry Phases Emerging from the $\pi$-Flux State: Competition between Antiferromagnetism and the Valence-Bond-Solid State, Phys. Rev. Lett. 95, 036402 (2005). 
[29] T. Senthil and M. P. A. Fisher, Competing Orders, Nonlinear Sigma Models, and Topological Terms in Quantum Magnets, Phys. Rev. B 74, 064405 (2006).

[30] C. Wang and T. Senthil, Dual Dirac Liquid on the Surface of the Electron Topological Insulator, Phys. Rev. X 5, 041031 (2015).

[31] M. A. Metlitski and A. Vishwanath, Particle-Vortex Duality of Two-Dimensional Dirac Fermion from Electric-Magnetic Duality of Three-Dimensional Topological Insulators, Phys. Rev. B 93, 245151 (2016).

[32] D. F. Mross, J. Alicea, and O. I. Motrunich, Explicit Derivation of Duality between a Free Dirac Cone and Quantum Electrodynamics in $(2+1)$ Dimensions, Phys. Rev. Lett. 117, 016802 (2016).

[33] C. Xu and Y.-Z. You, Self-Dual Quantum Electrodynamics as Boundary State of the Three-Dimensional Bosonic Topological Insulator, Phys. Rev. B 92, 220416 (2015).

[34] N. Seiberg, T. Senthil, C. Wang, and E. Witten, A Duality Web in Dimensions and Condensed Matter Physics, Ann. Phys. (Amsterdam) 374, 395 (2016).

[35] A. Karch and D. Tong, Particle-Vortex Duality from 3D Bosonization, Phys. Rev. X 6, 031043 (2016).

[36] J. Murugan and H. Nastase, Particle-Vortex Duality in Topological Insulators and Superconductors, J. High Energy Phys. 05 (2017) 159.

[37] P.-S. Hsin and N. Seiberg, Level/Rank Duality and Chern-Simons-Matter Theories, J. High Energy Phys. 09 (2016) 095.

[38] C. Wang and T. Senthil, Time-Reversal Symmetric U(1) Quantum Spin Liquids, Phys. Rev. X 6, 011034 (2016).

[39] M. A. Metlitski, $S$-Duality of $u(1)$ Gauge Theory with $\theta=\pi$ on Non-Orientable Manifolds: Applications to Topological Insulators and Superconductors, arXiv:1510.05663.

[40] D. T. Son, Is the Composite Fermion a Dirac Particle?, Phys. Rev. X 5, 031027 (2015).

[41] C. Wang and T. Senthil, Half-Filled Landau Level, Topological Insulator Surfaces, and Three-Dimensional Quantum Spin Liquids, Phys. Rev. B 93, 085110 (2016).

[42] S. D. Geraedts, M. P. Zaletel, R. S. K. Mong, M. A. Metlitski, A. Vishwanath, and O. I. Motrunich, The Half-Filled Landau Level: The Case for Dirac Composite Fermions, Science 352, 197 (2016).

[43] O. Aharony, Baryons, Monopoles and Dualities in Chern-Simons-Matter Theories, J. High Energy Phys. 02 (2016) 93.

[44] O. Aharony, N. Seiberg, and Y. Tachikawa, Reading between the Lines of Four-Dimensional Gauge Theories, J. High Energy Phys. 08 (2013) 115.

[45] N. Karthik and R. Narayanan, Scale Invariance of ParityInvariant Three-Dimensional QED, Phys. Rev. D 94, 065026 (2016).

[46] K. Slagle, Y.-Z. You, and C. Xu, Exotic Quantum Phase Transitions of Strongly Interacting Topological Insulators, Phys. Rev. B 91, 115121 (2015).

[47] Y.-Y. He, H.-Q. Wu, Y.-Z. You, C. Xu, Z. Y. Meng, and Z.-Y. Lu, Bona Fide Interaction-Driven Topological Phase Transition in Correlated Symmetry-Protected Topological States, Phys. Rev. B 93, 115150 (2016).
[48] S. J. Hands, J. B. Kogut, L. Scorzato, and C. G. Strouthos, Noncompact Three-Dimensional Quantum Electrodynamics with $N_{f}=1$ and $N_{f}=4$, Phys. Rev. B 70, 104501 (2004).

[49] A. Kapustin and M. J. Strassler, On Mirror Symmetry in Three Dimensional Abelian Gauge Theories, J. High Energy Phys. 04 (1999) 021.

[50] E. Witten, SL(2,Z) Action On Three-Dimensional Conformal Field Theories with Abelian Symmetry, arXiv: hep-th/0307041.

[51] Though $z_{\alpha}$ transforms as a spinor under $\mathrm{SU}(2)$, rotations in the center of SU(2) can be compensated by a U(1) gauge transformation so that the spin-rotation symmetry of the model is $\left\{[\mathrm{SU}(2)] / Z_{2}\right\}=\mathrm{SO}(3)$.

[52] It is customary in condensed-matter literature to call a U(1) gauge field "noncompact" if its flux is conserved. In highenergy literature, a U(1) gauge field is called "compact" if monopole (instanton) operators are local. In this paper, we use the condensed-matter notation, and call our gauge fields noncompact. Of course, they are also compact in the high-energy sense; i.e., monopole operators can, in principle, be added to the action.

[53] O. I. Motrunich and A. Vishwanath, Emergent Photons and Transitions in the O(3) Sigma Model with Hedgehog Suppression, Phys. Rev. B 70, 075104 (2004).

[54] F. D. M. Haldane, $O(3)$ Nonlinear $\sigma$ Model and the Topological Distinction between Integer- and HalfInteger-Spin Antiferromagnets in Two Dimensions, Phys. Rev. Lett. 61, 1029 (1988).

[55] N. Read and S. Sachdev, Spin-Peierls, Valence-Bond Solid, and Néel Ground States of Low-Dimensional Quantum Antiferromagnets, Phys. Rev. B 42, 4568 (1990).

[56] M. Levin and T. Senthil, Deconfined Quantum Criticality and Néel Order via Dimer Disorder, Phys. Rev. B 70, 220403 (2004).

[57] Specifically, we need to look for a power-law correlated fixed point that has only one relevant perturbation that breaks $\mathrm{SO}(5)$ to $\mathrm{SO}(3) \times \mathrm{O}(2)$ (or rather the symmetry of the lattice model, which is slightly smaller). This perturbation will correspond to the operator whose coefficient is tuned to place the lattice model at its critical point.

[58] A. Vishwanath and T. Senthil, Physics of Three-Dimensional Bosonic Topological Insulators: Surface-Deconfined Criticality and Quantized Magnetoelectric Effect, Phys. Rev. X 3, 011016 (2013).

[59] Here, we use a more "traditional" procedure of defining a Dirac fermion action. Namely, a single Dirac fermion should come together with a Chern-Simons term at level $k= \pm 1 / 2$ to avoid a gauge anomaly. A more precise way to define this theory is to use the procedure in Refs. [34,60], where the partition function of a massless Dirac fermion is written as $Z_{\psi}=\left|Z_{\psi}\right| e^{-i \pi \eta[A, g] / 2}$, where $A$ is the gauge field, either dynamical or background, and $g$ is the spacetime metric. $\eta$ is defined in terms of eigenvalues of the Dirac operator [60]; see Eq. (116). This form corresponds to UV completing the massless Dirac theory by adding two extra "heavy" Dirac fermions with the same sign of Dirac mass. This enables retaining flavor $\mathrm{SU}(2)$ rotations as an exact symmetry. If we do not care about this $\mathrm{SU}(2)$ symmetry, then we can choose the two heavy fermions to have 
opposite masses, in which case the partition function is real. Further, strictly speaking, $A$ should be regarded as a $\operatorname{spin}_{c}$ connection and not an ordinary U(1) gauge field, which means that fields with odd charge are fermions [39]. The more precise form of the Lagrangian is presented in detail in Appendix A.

[60] E. Witten, Fermion Path Integrals and Topological Phases, Rev. Mod. Phys. 88, 035001 (2016).

[61] Note that the fermions themselves transform as spinors under the flavor $\mathrm{SU}(2)$, but rotations by the element of the center $Z_{2}$ can be compensated by a U(1) gauge rotation. This might naively lead to the expectation that the global symmetry, excluding charge conjugation, is $\mathrm{SO}(3) \times \mathrm{U}(1)$. However, the theory has monopole operators that are local and that transform as spinors under the SU(2). Rotations by the center of SU(2) on these can now be compensated by $\pi$ rotations under the flux conservation global U(1), and therefore the global symmetry apparent in the Lagrangian, excluding charge conjugation, is $\left\{[\mathrm{SU}(2) \times \mathrm{U}(1)] / Z_{2}\right\}$.

[62] Including charge conjugation gives $\left[\mathrm{SU}(2) \times \mathrm{Pin}(2)_{-}\right] / Z_{2}$. The notation Pin (2)_ means that the charge-conjugation operation, which reverses the global U(1) charge, squares to the -1 element of $\mathrm{U}(1)$.

[63] The full manifest symmetry in this case is $\left[\operatorname{Pin}(2)_{-} \times \operatorname{Pin}(2)_{-}\right] / Z_{2}$.

[64] Strictly speaking, we take $B+B^{\prime}$ and $B-B^{\prime}$ to be properly quantized $\mathrm{U}(1)$ gauge fields.

[65] V. Borokhov, A. Kapustin, and X. Wu, Topological Disorder Operators in Three-Dimensional Conformal Field Theory, J. High Energy Phys., 112002049.

[66] A brief review of some math concepts relevant to the information in Sec. VII is given in Appendix D.

[67] F. Benini, P.-S. Hsin, and N. Seiberg, Comments on Global Symmetries, Anomalies, and Duality in $(2+1) \mathrm{d}$, J. High Energy Phys. 04 (2017) 135.

[68] Strictly speaking, $a$ is a $\operatorname{spin}_{c}$ connection.

[69] A. B. Kuklov, N. V. Prokof'ev, B. V. Svistunov, and M. Troyer, Deconfined Criticality, Runaway Flow in the Two-Component Scalar Electrodynamics and Weak First-Order Superfluid-Solid Transitions, Ann. Phys. (Amsterdam) 321, 1602 (2006).

[70] S. Kragset, E. Smørgrav, J. Hove, F. S. Nogueira, and A. Sudbø, First-Order Phase Transition in Easy-Plane Quantum Antiferromagnets, Phys. Rev. Lett. 97, 247201 (2006).

[71] J. D'Emidio and R. K. Kaul, First-Order Superfluid to Valence-Bond Solid Phase Transitions in Easy-Plane $\mathrm{SU}(n)$ Magnets for Small n, Phys. Rev. B 93, 054406 (2016).

[72] J. D'Emidio and R. K. Kaul, New Easy-Plane $\mathbb{C P}^{N-1}$ Fixed Points, Phys. Rev. Lett. 118, 187202 (2017).

[73] In Refs. [46,47], the quantum phase transition was discussed with the $\mathrm{O}(4)$ nonlinear sigma model with a topological term, which according to Ref. [29] is the low-energy effective field theory of the $N_{f}=2 \mathrm{QED}_{3}$.

[74] W. Chen, M. P. A. Fisher, and Y.-S. Wu, Mott Transition in an Anyon Gas, Phys. Rev. B 48, 13749 (1993).

[75] M. Barkeshli and J. McGreevy, A Continuous Transition between Fractional Quantum Hall and Superfluid States, Phys. Rev. B 89, 235116 (2014).
[76] In the quantum magnetism realization these are simply the Néel and VBS ordered states.

[77] Y.-M. Lu and A. Vishwanath, Theory and Classification of Interacting Integer Topological Phases in Two Dimensions: A Chern-Simons Approach, Phys. Rev. B 86, 125119 (2012).

[78] T. Senthil and M. Levin, Integer Quantum Hall Effect for Bosons, Phys. Rev. Lett. 110, 046801 (2013).

[79] T. Grover and A. Vishwanath, Quantum Phase Transition between Integer Quantum Hall States of Bosons, Phys. Rev. B 87, 045129 (2013).

[80] Y.-M. Lu and D.-H. Lee, Quantum Phase Transitions between Bosonic Symmetry-Protected Topological Phases in Two Dimensions: Emergent $\mathrm{QED}_{3}$ and Anyon Superfluid, Phys. Rev. B 89, 195143 (2014).

[81] Y. Fuji, Y.-C. He, S. Bhattacharjee, and F. Pollmann, Bridging Coupled Wires and Lattice Hamiltonian for Two-Component Bosonic Quantum Hall States, Phys. Rev. B 93, 195143 (2016).

[82] Here, $Z_{2}^{T}$ refers to time reversal, which is antiunitary.

[83] M. Cheng and C. Xu, Series of $(2+1)$-Dimensional Stable Self-Dual Interacting Conformal Field Theories, Phys. Rev. B 94, 214415 (2016).

[84] To be completely precise, Ref. [37] stated that the enhanced symmetry is $\operatorname{Spin}(4)$, while according to our discussion it is $\mathrm{SO}(4)$. In particular, we will not find local operators transforming under the $\left(\frac{1}{2}, 0\right)$ or $\left(0, \frac{1}{2}\right)$ representations.

[85] In fact, for the lattice antiferromagnet another symmetrybreaking term is allowed: $\left(\partial_{x} n_{1}\right)^{2}+\left(\partial_{y} n_{2}\right)^{2}+\cdots$ (see also note in Sec. IV D). (This operator lives in a spin-2 representation of spatial rotations; for a 3D CFT, unitarity bounds indicate that spin-2 operators have RG eigenvalue $\leq 0$.) This perturbation is absent for the continuum easyplane $\mathrm{NCCP}^{1}$ field theory, where the VBS U(1) is an exact internal symmetry.

[86] Strictly speaking, the anisotropy term dual to the Ising mass should be $\lambda\left[\left|z_{1}\right|^{2}\left|z_{2}\right|^{2}-\alpha\left(\left|z_{1}\right|^{2}+\left|z_{2}\right|^{2}\right)\right]$, where the second term is added to keep the theory critical when $\lambda>0$. We discuss this further in Sec. IV E.

[87] Strictly speaking, when $\lambda>0$, we need to compensate the anisotropy term with a mass term, $-\alpha \lambda\left(\left|z_{1}\right|^{2}+\left|z_{2}\right|^{2}\right)$ (likewise for the $w$ theory), to keep the theories on both sides critical. We discuss this further in Sec. IV E.

[88] See Ref. [15] for numerical results for correlation functions of $X^{(2)}$ components.

[89] For the quantum antiferromagnet we are also allowed the term $\left(\partial_{x} n_{1}\right)^{2}+\left(\partial_{y} n_{2}\right)^{2}+\cdots$. The emergence of $\mathrm{U}(1)$ symmetry for the VBS in JQ model simulations $[6,8]$ implies that this term is also irrelevant. This term is absent for the model of Refs. [14,15] since this model is isotropic in spacetime.

[90] Components of $X^{(4)}$ in the notation of Sec. IV D. These are presumed to be irrelevant at the $S U(2)$ critical point, but are relevant in the $\mathrm{O}(3)$-breaking phase.

[91] See Ref. [15] for a direct numerical test of this using correlation functions.

[92] Z. Bi, A. Rasmussen, K. Slagle, and C. Xu, Classification and Description of Bosonic Symmetry Protected Topological Phases with Semiclassical Nonlinear Sigma Models, Phys. Rev. B 91, 134404 (2015). 
[93] We use the periodicity of the physics under shifts $\Theta \rightarrow \Theta+2 \pi$, which holds for this system.

[94] X.-G. Wen, Quantum Field Theory of Many-Body Systems: From the Origin of Sound to an Origin of Light and Electrons, Oxford Graduate Texts (Oxford University Press, New York, 2004).

[95] The $\mathrm{QED}_{3}$ theory we discuss here is not to be confused with the "staggered flux state" known in the spin-liquid literature [94] (which is also described by $\mathrm{QED}_{3}$ with four Dirac fermions at low energy)-lattice symmetries act in distinct ways in these two theories.

[96] A. G. Abanov and P. B. Wiegmann, Theta-Terms in Nonlinear Sigma-Models, Nucl. Phys. B570, 685 (2000).

[97] A formal but elegant formulation of this consideration is described in Sec. VII in terms of matching the second Stiefel-Whitney classes of the bundles corresponding to the background and dynamical gauge fields.

[98] For simplicity, we may define monopole statistics by making just the $\mathrm{SO}(2)$ part of the gauge group dynamical.

[99] M. A. Metlitski, C. L. Kane, and M. P. A. Fisher, Bosonic Topological Insulator in Three Dimensions and the Statistical Witten Effect, Phys. Rev. B 88, 035131 (2013).

[100] C. Wang and T. Senthil, Boson Topological Insulators: A Window into Highly Entangled Quantum Phases, Phys. Rev. B 87, 235122 (2013).

[101] For instance, if $X_{1,2}$ are $X$ fields from the two copies of Eq. (100), we can add an $\mathrm{SO}(5)$-symmetric mass term $m \operatorname{Tr}\left(\bar{X}_{1} X_{1}-\bar{X}_{2} X_{2}\right)$. Integrating $X_{1,2}$ out, we get a pure Yang-Mills theory for $a$ with no Chern-Simons term, which is expected to confine.

[102] J. Oon, G. Y. Cho, and C. Xu, Two-Dimensional Symmetry-Protected Topological Phases with $\operatorname{PSU}(N)$ and Time-Reversal Symmetry, Phys. Rev. B 88, 014425 (2013).

[103] C. Wang, A. Nahum, and T. Senthil, Topological Paramagnetism in Frustrated Spin-1 Mott Insulators, Phys. Rev. B 91, 195131 (2015).

[104] Though this parton construction made use of a $Z_{2}^{T}$ invariant fermion SPT, the final boson SPT is stable even if $Z_{2}^{T}$ is broken. As we discuss below, with full $\mathrm{SO}(5) \times Z_{2}^{T}$ symmetry the response to a background $\mathrm{SO}(5)$ gauge field has a discrete $\theta$ term, but not the conventional $\theta$ term of Eq. (109). If now $Z_{2}^{T}$ is broken but $S O(5)$ is preserved, then such a conventional theta term is allowed. Regardless, the presence of the discrete theta term means that we still have a nontrivial SPT phase. More physically, the $\mathrm{SO}(5)$ monopole which breaks $\mathrm{SO}(5)$ to $\mathrm{SO}(2) \times \mathrm{SO}(3)$ will still transform as a spinor under $\mathrm{SO}(3)$ but in the absence of $Z_{2}^{T}$ is allowed to have nonzero $\mathrm{SO}(2)$ charge.

[105] Strictly speaking, $\mathcal{A}^{5}$ is the connection on the $\mathrm{SO}(5)$ gauge bundle. Here, we abuse the notation a bit by using $\mathcal{A}^{5}$ to label both the bundle and the connection.

[106] C. Wang and T. Senthil, Interacting Fermionic Topological Insulators/Superconductors in Three Dimensions, Phys. Rev. B 89, 195124 (2014).

[107] C. Wang and T. Senthil, Composite Fermi Liquids in the Lowest Landau Level, Phys. Rev. B 94, 245107 (2016).

[108] I. Sodemann, I. Kimchi, C. Wang, and T. Senthil, Composite Fermion Duality for Half-Filled Multicomponent Landau Levels, Phys. Rev. B 95, 085135 (2017).
[109] Of course, if the ray ends at a gapless CFT where allowed $\mathrm{SO}(5)$-breaking terms are relevant, these terms will eventually become large and the above constraints will not apply.

[110] As usual, we transition to Euclidean spacetime here. Furthermore, we formulate the theory on an arbitrary spacetime manifold $M$. The ability to do so is a useful formal consistency check on the theory. We assume that the manifold $M$ is oriented; i.e., unless otherwise noted, we do not discuss "gauging" of time-reversal symmetry.

[111] M. Nakahara, Geometry, Topology and Physics, Graduate Student Series in Physics (Taylor \& Francis group, New York, 2003).

[112] The subscript $T M$ on $\operatorname{Spin}(3)$ reminds us that these are transition functions associated with the tangent bundle.

[113] Here, $\omega_{\mu}$ is the spin connection, which we include for further generality to describe curved manifolds [114].

[114] T. Eguchi, P. B. Gilkey, and A. J. Hanson, Gravitation, Gauge Theories and Differential Geometry, Phys. Rep. 66, 213 (1980).

[115] Strictly speaking, to show that $T$ is nonanomalous we have to regulate the theory on a nonorientable manifold. However, this can be done as the mass term $\chi^{T} \tau^{y} \mu^{z} \chi$ preserves $T$-we can introduce a PV regulator with this mass term.

[116] Level 1 is defined so that for an $\mathrm{SO}(2)$ subgroup acting on the first two components of an $n$ vector, $\sigma_{x y}=1$. In other words, level 1 corresponds to the $\mathrm{SO}(n)$ response of $n$ identical copies of a $p_{x}+i p_{y}$ superconductor.

[117] For an $\mathrm{SO}(n)$ gauge field the index is always even as $\left[C K, i D_{A}\right]=0$, and $(C K)^{2}=-1$, with $C$ the chargeconjugation operator and $K$ the complex conjugation.

[118] In the case when $M$ is supplemented with a spin structure, which is preserved by the extension, $\mathrm{CS}_{\mathrm{SO}(n)}\left[A, Y_{4}\right]$ and $\mathrm{CS}_{g}\left[Y_{4}\right]$ are separately independent of the extension.

[119] In this section, we are slightly sloppy and often use $A$ to label both the bundle and the connection. Moreover, we use $A^{s}$ to label both the $\mathrm{SO}(3)_{s}$ bundle and the $\mathrm{SU}(2)_{s}$ lift. The meaning is clear from the context. Similarly, $A^{5}$ denotes both the $\mathrm{SO}(5)$ bundle and the $\mathrm{Sp}(4)$ lift.

[120] J. Milnor and J. Stasheff, Characteristic Classes (Princeton University Press, Princeton, NJ, 1974).

[121] There is a theorem that on any closed four-manifold, $w_{2}\left(T Y_{4}\right)$ can be lifted to an integer cohomology class $\hat{w}_{2} \in H^{2}(Z)$. (This is the statement that every four-mani-

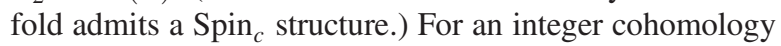
class $a, \mathcal{P}(a)=a \cup a$. So we need to show $\int_{Y_{4}} \hat{w}_{2} \cup \hat{w}_{2}=$ $\sigma(\bmod 4)$. Letting the unimodular matrix $Q$ be the intersection pairing on Free $\left(H^{2}\left(Y_{4}, Z\right)\right)$, we must show $\left(\hat{w}_{2}\right)^{T} Q \hat{w}_{2}=\sigma(Q)(\bmod 4)$, where $\sigma(Q)$ is the signature of the matrix $Q$. Actually, a stronger statement $\left(\hat{w}_{2}\right)^{T} Q \hat{w}_{2}=$ $\sigma(Q)$ (mod 8) holds and can be derived purely from algebra (see Ref. [122], p. 24). Actually, this statement is familiar to users of the APS theorem. Consider a general $\operatorname{Spin}_{c}$ connection with field strength $F$ on a four-manifold. The Atiyah-Singer theorem [111,114] then tells us that $\frac{1}{2} \int[F /(2 \pi)] \wedge[F /(2 \pi)]-[\sigma / 8] \in Z$. Now a $\operatorname{Spin}_{c}$ connection has $\int[F /(2 \pi)]=\int\left(\hat{w}_{2} / 2\right)(\bmod Z)$ for any oriented two-cycle. Choosing a special case where $[F /(2 \pi)]=\left(\hat{w}_{2} / 2\right)$, we obtain the needed result. 
[122] J. Milnor and D. Husemoller, Symmetric Bilinear Forms (Springer-Verlag, Berlin, 1973).

[123] There is also one caveat here: does an extension always exist? For this, one must calculate the 3D bordism groupa task that we do not attempt here.

[124] Below, we are somewhat cavalier using $[F /(2 \pi)]$ to denote both the Chern class [in $\left.H^{2}(Z)\right]$ and the field strength $F=d A$ of a $\mathrm{U}(1)$ bundle

[125] One way to obtain this result is the following. Recall that the Haldane chain is described by the $\mathrm{O}(3)$ nonlinear sigma model at $\theta=2 \pi$. In a $C P^{1}$ description the Lagrangian is [126] $\mathcal{L}=\left|\left(\partial_{\mu}-i a_{\mu}\right) z\right|^{2}+i \theta f / 2 \pi$, where $f$ is the field strength associated with $a_{\mu}=-i z^{\dagger} \partial_{\mu} z$. A background $\mathrm{SO}(3)_{s}$ gauge field translates into a background $\mathrm{SU}(2)_{s}$ gauge field for the $z$ field. If, however, the $\mathrm{SO}(3)_{s}$ gauge bundle has $w_{2} \neq 0$, we cannot then naively lift it to $\mathrm{SU}(2)_{s}$. However, $z$ is also coupled to the $\mathrm{U}(1)$ gauge field $a$. Any defect in the cocycle condition of the $\mathrm{SO}(3)_{s}$ bundle can be compensated by a $\pi$ flux of the U(1) gauge field. Thus, a configuration of the $\mathrm{SO}(3)_{s}$ gauge field with a nonzero $w_{2}$ necessarily induces a background $a_{\mu}$ configuration where $\int_{\Sigma} \frac{f}{2 \pi}=\int_{\Sigma}\left\{\left[w_{2}\left(A^{s}\right)\right] / 2\right\}(\bmod \mathrm{Z})$. For the Haldane chain, from the $C P^{1}$ Lagrangian with the $\theta$ term at $\theta=2 \pi$, we immediately see that the partition function has an extra phase $e^{i \pi \int_{\Sigma} w_{2}\left[A^{s}\right]}$. Clearly, on a closed manifold this is invariant under "gauge transformations," $w_{2} \rightarrow w_{2}+d n$. However, in the presence of a boundary this is no longer true. Gauge invariance can be mended by including a Wilson line $W\left[A^{s}\right]$ in the spin-1/2 representation along the boundary; i.e., the partition function now becomes $W\left[A^{s}\right](-1) \int_{\Sigma} w_{2}$. This boundary Wilson line is precisely the well-known dangling spin-1/2 moment at the boundary of the Haldane chain.

[126] E. Witten, Instatons, the Quark Model and the 1/n Expansion, Nucl. Phys. B149, 285 (1979).

[127] S. El-Showk, M. F. Paulos, D. Poland, S. Rychkov, D. Simmons-Duffin, and A. Vichi, Solving the $3 D$ Ising Model with the Conformal Bootstrap, Phys. Rev. D 86, 025022 (2012).

[128] F. Kos, D. Poland, and D. Simmons-Duffin, Bootstrapping the $\mathrm{O}(N)$ Vector Models, J. High Energy Phys. 06 (2014) 091.

[129] S. M. Chester and S.S. Pufu, Towards Bootstrapping $\mathrm{QED}_{3}$, J. High Energy Phys. 08 (2016) 019.

[130] J. A. Gracey, Electron Mass Anomalous Dimension at $O\left(1 / n_{f}^{2}\right)$ in Quantum Electrodynamics, Phys. Lett. B 317, 415 (1993).

[131] E. Dyer, M. Mezei, and S. S. Pufu, Monopole Taxonomy in Three-Dimensional Conformal Field Theories, arXiv: 1309.1160.

[132] S. M. Chester and S. S. Pufu, Anomalous Dimensions of Scalar Operators in $\mathrm{QED}_{3}$, J. High Energy Phys. 08 (2016) 069.

[133] Since the microscopic model has an exact $\mathrm{SO}(4)$ symmetry here, for $\mathrm{QED}_{3}$ to describe it, the $\mathrm{SO}(4)$ symmetry must necessarily emerge at the critical point.

[134] These include the JQ model [6-13] and a related loop model [14,15], lattice [16,17] and continuum [18] field theories, and the classical 3D dimer model [19-22], which has different microscopic symmetries to the JQ model but has been argued to have the same continuum description. See also numerical work on generalizations to $\mathrm{SU}(n)$ with $n>2$ [135-138].

[135] K. S. D. Beach, F. Alet, M. Mambrini, and S. Capponi, $\mathrm{SU}(n)$ Heisenberg Model on the Square Lattice: A Continuous-n Quantum Monte Carlo Study, Phys. Rev. B 80, 184401 (2009).

[136] A. Banerjee, K. Damle, and F. Alet, Impurity Spin Texture at the Critical Point between Néel-Ordered and ValenceBond-Solid States in Two-Dimensional SU(3) Quantum Antiferromagnets, Phys. Rev. B 83, 235111 (2011).

[137] R. K. Kaul, Quantum Criticality in SU(3) and SU(4) Antiferromagnets, Phys. Rev. B 84, 054407 (2011).

[138] R. K. Kaul and A. W. Sandvik, Lattice Model for the SU(n) Néel to Valence-Bond Solid Quantum Phase Transition at Large n, Phys. Rev. Lett. 108, 137201 (2012).

[139] For example, in models for the Néel-VBS transition there is a single direct transition between the two phases, the expected U(1) symmetry for the VBS order parameter indeed emerges at the critical point, and the Néel vector has a large anomalous dimension, which was predicted as a signature of deconfinement.

[140] Some features of the numerical results are suggestive of this. See, e.g., the discussion of correlation functions in Ref. [14], and the fits in Ref. [24].

[141] H. Suwa, A. Sen, and A. W. Sandvik, Level Spectroscopy in a Two-Dimensional Quantum Magnet: Linearly Dispersing Spinons at the Deconfined Quantum Critical Point, Phys. Rev. B 94, 144416 (2016).

[142] A. W. Sandvik, Finite-Size Scaling and Boundary Effects in Two-Dimensional Valence-Bond Solids, Phys. Rev. B 85, 134407 (2012).

[143] To describe the (generic) deconfined critical point, the SO(5) CFT should have no relevant operator that is invariant under all symmetries; see Sec. IV D. The bootstrap result constrains the $\mathrm{SO}(5)$ vector's anomalous dimension, under the assumption that there is no relevant symmetry-trivial operator.

[144] Take for example the $q$-state Potts model in 3D, with a large value of $q$. The transition is generically first order due to a cubic invariant in the Landau-Ginsburg action. One could fine-tune the couplings to be close to the free fixed point, giving a very weak first-order transition. This mechanism is nongeneric, and is not the mechanism discussed in the text.

[145] B. Nienhuis, A. N. Berker, E. K. Riedel, and M. Schick, First- and Second-Order Phase Transitions in Potts Models: Renormalization-Group Solution, Phys. Rev. Lett. 43, 737 (1979).

[146] M. Nauenberg and D. J. Scalapino, Singularities and Scaling Functions at the Potts-Model Multicritical Point, Phys. Rev. Lett. 44, 837 (1980).

[147] J. L. Cardy, M. Nauenberg, and D. J. Scalapino, Scaling Theory of the Potts-Model Multicritical Point, Phys. Rev. B 22, 2560 (1980).

[148] We may see this in more detail using the logic of Refs. [147,149]. Starting with the RG equations for $\lambda$ and for the leading irrelevant coupling $g$ at $\tau=\tau_{c}$, expand in $\Delta^{2}=\tau-\tau_{c}$ assuming analyticity in $\Delta^{2}$ and the couplings. 
Using the freedom to make analytic redefinitions of the couplings gives Eq. (156) and $d g / d t=-(y+m \lambda) g$, neglecting subleading corrections ( $t$ is RG time). The zero of the latter equation at $g=0$ is preserved to all orders in $\Delta^{2}$. We have $\lambda(t)=-\Delta \tan \Delta\left[t-t_{*}\right]$, with $t_{*} \simeq$ $\pi / 2 \Delta-1 / \lambda(0)$. The correlation length is determined by setting $\lambda \sim-1$, giving $\ln \xi=\pi / \Delta+\mathrm{O}(1)$. The subleading coupling behaves as $g(t)=g_{0} e^{-y t}\left\{\left[\Delta^{2}+\lambda(t)^{2}\right] /\right.$ $\left.\left[\Delta^{2}+\lambda(0)^{2}\right]\right\}^{m / 2}$. Once the RG time is of order $1 / \Delta, g(t)$ has become exponentially small in $1 / \Delta$. On RG time scales of this order the typical variation in $\lambda(t)$, and therefore the typical quasiuniversal drift in large scale properties, is $\mathrm{O}(\Delta)$.

[149] F. J. Wegner, in Phase Transitions and Critical Phenomena, edited by C. Domb and M. S. Green (Academic Press, London, 1976), Vol. 6, Chap. 2.

[150] R. J. Baxter, Potts Model at the Critical Temperature, J. Phys. C 6, L445 (1973).

[151] J. Lee and J. M. Kosterlitz, New Numerical Method to Study Phase Transitions, Phys. Rev. Lett. 65, 137 (1990).

[152] A. Klümper, A. Schadschneider, and J. Zittartz, Inversion Relations, Phase Transitions and Transfer Matrix Excitations for Special Spin Models in Two Dimensions, Z. Phys. B 76, 247 (1989).

[153] E. Buffenoir and S. Wallon, The Correlation Length of the Potts Model at the First-Order Transition Point, J. Phys. A 26, 3045 (1993).

[154] H. Gies and J. Jaeckel, Chiral Phase Structure of QCD with Many Flavors, Eur. Phys. J. C 46, 433 (2006).

[155] D. B. Kaplan, J.-W. Lee, D. T. Son, and M. A. Stephanov, Conformality Lost, Phys. Rev. D 80, 125005 (2009).

[156] S. Gukov, RG Flows and Bifurcations, Nucl. Phys. B919, 583 (2017).

[157] S. Giombi, I. R. Klebanov, and G. Tarnopolsky, Conformal $\mathrm{QED}_{d}$, F-Theorem and the $\epsilon$ Expansion, J. Phys. A 49, 135403 (2016).

[158] I. F. Herbut, Chiral Symmetry Breaking in ThreeDimensional Quantum Electrodynamics as Fixed Point Annihilation, Phys. Rev. D 94, 025036 (2016).

[159] A. Nahum, J. T. Chalker, P. Serna, M. Ortuño, and A. M. Somoza, Phase Transitions in Three-Dimensional Loop Models and the $c P^{n-1}$ Sigma Model, Phys. Rev. B 88, 134411 (2013).

[160] A fixed point annihilation phenomenon also occurs in the so-called "compact" $C P^{n-1}$ model [an $\mathrm{SU}(n)$-symmetric Landau-Ginsburg theory obtained from $\mathrm{NCCP}^{n-1}$ by condensing the strength-1 monopole]. However, there the critical and tricritical fixed points annihilate when $n$ is increased (with $n_{c} \sim 3$ ) rather than when $n$ is decreased, as is the case for $\mathrm{NCCP}^{n-1}$ [159].

[161] It is clear from the drift in effective critical exponents that current simulations are not in this regime (which would show simple exponent values).
[162] In this and the next Appendix, we use the precise definition of the Dirac partition function in terms of the $\eta$ invariant. We also include a coupling to a background spacetime metric $g$.

[163] We have not shown explicitly background SU(2) gauge fields - they can be incorporated if needed.

[164] A. C. Potter, C. Wang, M. A. Metlitski, and A. Vishwanath, Realizing Topological Surface States in a LowerDimensional Flat Band, arXiv:1609.08618.

[165] C. P. Burgess and B. P. Dolan, Particle-Vortex Duality and the Modular Group: Applications to the Quantum Hall Effect and Other Two-Dimensional Systems, Phys. Rev. B 63, 155309 (2001).

[166] J. L. Cardy and E. Rabinovici, Phase Structure of $z_{p}$ Models in the Presence of a $\theta$ Parameter, Nucl. Phys. B205, 1 (1982).

[167] J. L. Cardy, Duality and the $\theta$ Parameter in Abelian Lattice Models, Nucl. Phys. B205, 17 (1982).

[168] E. Fradkin and S. Kivelson, Modular Invariance, SelfDuality and the Phase Transition between Quantum Hall Plateaus, Nucl. Phys. B474, 543 (1996).

[169] Here, the operation $S^{2}=-1$ corresponds to changing the sign of the gauge coupling.

[170] This limit is where the gauge coupling is much weaker than the quartic terms (so that the RG flow comes very close to the ungauged Wilson-Fisher fixed point before the gauge coupling drives it away). Also, in this example the bare quartic terms do not couple the two fields.

[171] Note though that if we assume the reliability of the $S$ operation, then we can infer the existence of a CFT with the same symmetries as $\mathrm{NCCP}^{1}$. It should be noted, however, that the formal construction says nothing about the stability (number of relevant perturbations) of this CFT.

[172] An instructive example is to take $\mathrm{CFT}_{1}$ to be a trivial theory with partition function $Z_{\mathrm{CFT}_{1}}=1$. In a condensedmatter context this is the fixed point theory for a bosonic Mott insulator. If we now couple the bosons to a dynamical gauge field to obtain $\tilde{Z}_{S}$, we see that the new theory is in a phase where the new global U(1) symmetry associated with conservation of $d A$ is spontaneously broken (a superfluid). If we use the formal definition in Eq. (C1), we get $Z_{S}[B]=\delta(B)$, as expected for a superfluid. In a formal sense this partition function is conformally invariant-we may think of it as the fixed point description of a superfluid phase obtained by taking the phase stiffness of the Goldstone mode to $\infty$. Of course, $Z_{S}$ is physically lacking as it misses the Goldstone physics.

[173] A. Kapustin and R. Thorngren, Topological Field Theory on a Lattice, Discrete Theta-Angles and Confinement, arXiv:1308.2926.

[174] Note that $\eta$ in Eq. (116) includes a contribution $N_{0}$ from the zero modes $\lambda=0$. If there are any zero modes, then the absolute value of the partition function $|Z(m=0)|$ vanishes and the phase is irrelevant.

[175] $i A_{\mu}$ is real, so crucially, $\left[i D_{A}, C K\right]=0$ still holds. 